\title{
Resource Allocation in Autonomous Cellular Networks
}

by

\author{
Bijan Golkar
}

A thesis submitted in conformity with the requirements

for the degree of Doctor of Philosophy

Graduate Department of Electrical and Computer Engineering

University of Toronto

(C) Copyright 2013 by Bijan Golkar 


\begin{abstract}
Resource Allocation in Autonomous Cellular Networks

Bijan Golkar

Doctor of Philosophy

Graduate Department of Electrical and Computer Engineering

University of Toronto

2013
\end{abstract}

With a fixed number of terminals and a fixed system spectrum, it can be shown that increasing the number of BSs in a cellular network results in a better network performance. However, a regular organized deployment of a cellular network with small cells is both prohibitively expensive and complicated. A possible solution is to allow the network to grow in an organic fashion. This calls for self-configurable approaches for resource allocation which not only improve the performance but also decrease the network planning as well as maintenance costs. Over the past few years, a particular type of small BSs known as femto-BSs has attracted tremendous attention from the industry and academia alike. Femto BSs operate on the licensed spectrum allocated to the cellular network. They are small, inexpensive and transmit at low power levels. Although the deployment of these small BSs result in significant improvements in the indoor coverage for both voice and data services, they only satisfy the immediate needs of the present cellular networks namely the improvement of the indoor coverage. They do not provide a fundamental solution for a scalable architecture which can organically grow and adapt to the short and long term changes in the network.

In this thesis, we propose a practical unified framework for the system design and resource allocation of cellular networks with various types of base stations (with different power budgets) deployed in an irregular fashion. We refer to these networks as autonomous cellular networks. To this end, we first introduce an adaptive localized ap- 
proach to resource allocation in traditional cellular networks. We show that localized resource assignment at the cost of partial loss of orthogonality at the cell edges can provide significant gains in the network performance. In the second part of the thesis, we propose an adaptive self-configurable resource allocation framework for cellular networks. A network clustering technique is proposed which enables coordinated resource allocation in a network with irregular deployment of BSs. The performance of the proposed framework is evaluated with the regular as well as the irregular deployment of BSs. 


\section{Dedication}

To my aunt 


\section{Acknowledgements}

I would like to express my most sincere gratitude to my supervisor, Professor Elvino

Sousa. His inspiring guidance full of encouragement and support turned the course of my PhD studies into a truly unique and memorable journey.

I am also grateful to the members of the $\mathrm{PhD}$ defence committee, Professor Ben Liang, Professor Shahrokh Valaee and Professor Sorin Voinigescu for their suggestions. The comments of the external examiner, Dr. Wen Tong, are also greatly appreciated.

This thesis is dedicated to my dearest aunt who passed away last year after a long and unyielding battle with cancer. I have always admired her strong and independent personality.

In the end, I would like to thank my parents and my brother for their constant love and support at every single stage of my life. I am and will always be indebted to the three of them for all my achievements in the past, the present and the future. 


\section{Contents}

1 Introduction $\quad 1$

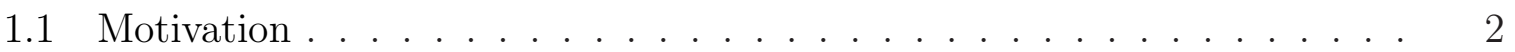

1.2 Contributions . . . . . . . . . . . . . . . . . . . 3

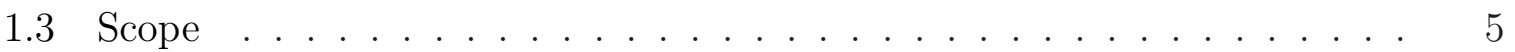

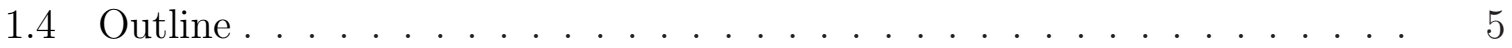

$\begin{array}{lll}2 & \text { Literature review and background material } & 7\end{array}$

2.1 Cellular network: A definition . . . . . . . . . . . . 7

2.2 Frequency reuse strategies . . . . . . . . . . . . . . . . . . 10

2.2.1 The traditional approach . . . . . . . . . . . . . . 10

2.2.2 Fractional frequency reuse . . . . . . . . . . . . . 11

2.3 Coordinated adaptive resource allocation . . . . . . . . . . . . 15

2.3.1 Two-level resource allocation . . . . . . . . . . . . . . . . 15

2.3.2 Adaptive FFR techniques . . . . . . . . . . . . . . 17

2.4 Resource allocation strategies and trade-offs . . . . . . . . . . . . . . . . 18

2.4.1 Proportional fairness . . . . . . . . . . . . . . . 19

2.5 Practical considerations . . . . . . . . . . . . . . 24

2.5.1 Scheduling granularity . . . . . . . . . . . . . . . 24

2.5.2 Channel model . . . . . . . . . . . . . . . . . . . . 25

2.5.3 Link spectral efficiency model . . . . . . . . . . . . . . 30 
2.5 .4 Performance metrics $\ldots \ldots \ldots \ldots$

3 Localized resource allocation 33

3.1 Scheduling cell . . . . . . . . . . . . . . . . . . . . . . . 33

3.2 Resource allocation granularity . . . . . . . . . . . . . 37

3.3 Resource allocation framework . . . . . . . . . . . . . . . . . . . 42

3.3 .1 Terminal grouping . . . . . . . . . . . . . . . . 42

3.3.2 Step 1 - User set resource allocation . . . . . . . . . . . . . 44

3.3.3 Power control strategy $\ldots \ldots \ldots \ldots$

3.3.4 Step 2 - Terminal scheduling . . . . . . . . . . . . . 48

3.3.5 Time scales of adaptation $\ldots \ldots \ldots \ldots \ldots$

3.4 Numerical results . . . . . . . . . . . . . . . . . . . . . . . . . 52

4 Autonomous cellular network $\quad 59$

4.1 Network shadow fading model . . . . . . . . . . . . . . . . . 62

4.1 .1 Background material . . . . . . . . . . . . . 63

4.1.2 Network correlation model . . . . . . . . . . . . . . . 66

4.1 .3 Cell radius . . . . . . . . . . . . . . . . . . . . . . . 71

4.1 .4 Outage probability . . . . . . . . . . . . 72

4.1.5 Correlation between two links with a common end . . . . . . . 74

4.2 Network clustering . . . . . . . . . . . . . . . . . . . 75

4.2 .1 Problem definition $\ldots \ldots \ldots \ldots \ldots \ldots$

4.2 .2 Similarity index . . . . . . . . . . . . . . . 79

4.2 .3 Clustering . . . . . . . . . . . . . . . 83

4.2.4 Coordinated resource allocation . . . . . . . . . . 90

4.3 Resource allocation framework . . . . . . . . . . . . . . . . 96

4.4 Numerical results . . . . . . . . . . . . . . . . . . . . . . . . 97

4.4 .1 Traditional cellular network . . . . . . . . . . . . 98 
4.4.2 Autonomous cellular network . . . . . . . . . . . . . . 103

5 Conclusion $\quad 110$

5.1 Summary . . . . . . . . . . . . . . . . . . 110

5.2 Future work . . . . . . . . . . . . . . . . . 112

$\begin{array}{ll}\text { Bibliography } & 112\end{array}$ 


\section{Chapter 1}

\section{Introduction}

The first public mobile telephone services were deployed in the late 1940s. In these early systems, a single high-powered transmitter, mounted on a large tower, covered distances of over 50 kilometers. The networks provided simultaneous service to a few subscribers. A higher demand directly translates into the need for a larger spectrum. Due to the scarcity of the spectrum, it was essential to develop an infrastructure which would support a large population of subscribers on a limited spectrum over large coverage areas. The cellular concept was proposed by AT\&T to the Federal Communications Commission (FCC) in 1968. It was a system level idea which proposed replacing a single, high power transmitter (large cell) with many low power transmitters (small cells). This became possible due to a smart frequency allocation strategy which allowed frequency reuse by reducing the co-channel interference to acceptable levels. One can claim that the cellular concept is

perhaps the most important breakthrough in the evolution of public mobile telephone services. In fact, despite all the technological improvements over the past couple of decades, the research in this field is still based on the cellular concept [28].

Partly due to the lack of technology, the first cellular networks were implemented in the late 1970s. The FCC allocated $40 \mathrm{MHz}$ of spectrum to the US Advanced Mobile Phones System (AMPS) in 1993 - the first generation of cellular networks. By the intro- 
duction of digital communications into cellular networks in the 1990s, several standards for the second generation of cellular networks were developed. The hardware for the US digital cellular system, under the Interim-Standard 54 (IS-54) and later IS-136, was installed in 1991 which supported three simultaneous users over a bandwidth of 30KHz. Qualcom Inc. introduced the CDMA cellular system (IS-95) in 1993, which supported 64 users orthogonally coded on a bandwidth of 1.25MHz. The Global System for Mobile (GSM) communications was introduced in 1994 which accommodated 8 simultaneous users in a bandwidth of $200 \mathrm{KHz}$. In the early 2000s, the third generation of cellular networks were developed mainly based on the CDMA technology. These networks provided unprecedented wireless services such as multi megabit Internet access, calls using Voice over Internet Protocol (VoIP), etc.

\subsection{Motivation}

Based on the cellular concept, increasing the number of base stations (BSs) at an extra deployment cost improves the network capacity with no requirement for additional radio spectrum. Accordingly, the coverage area of each BS has significantly reduced over the past couple of decades. In a typical modern urban cellular network, each BS covers an area with a radius in the range of $100-1000$ meters. Scaling down the size of each cell does not substantially affect the signal-to-interference-plus-noise ratio (SINR) level of each link. However, with the reduction of the cell coverage area, the number of served terminals per BS drops significantly. Each BS allocates more system resources to each terminal, which in turn results in a larger aggregate throughput for the end-user. The unrelenting increase in the demand for higher data rates requires further reductions of the cell radius. As an example, a ten-fold reduction of the size of each cell would require a very dense regular deployment of BSs (e.g. every 50 meters). Nonetheless, the deployment cost of such an infrastructure would be prohibitively high. More importantly, a dense deployment of BSs 
would not be practically feasible due to the physical limitations imposed by the urban infrastructure.

An alternative solution to satisfying the ever-growing service demand is the irregular deployment of small BSs:

Over the past few years, a particular type of small BSs known as femto BSs has attracted tremendous attention from the industry and academia alike. Femto BSs operate on the licensed spectrum allocated to the cellular network. They are small, inexpensive and transmit at low power levels. They are developed to be deployed indoors and have a back-haul connection to the network via a conventional digital subscriber line (DSL) or cable broadband access. The femto BS deployment results in significant savings for the network operator due to the offload of a substantial part of the (data) traffic onto the wire-line networks through the femto BSs [10,27]. The favorable economics together with the ease of deployment and significant improvements in the indoor coverage for both voice and data services have resulted in an increasing popularity of this concept in the recent years. Despite the clear advantages of the femto BSs, they only satisfy the immediate needs of the present cellular networks — namely the improvement of the indoor coverage. They do not provide a fundamental solution for a scalable architecture which can organically grow and adapt to the short and long term changes in the network. The work in the present dissertation has been conducted with such a vision.

\subsection{Contributions}

A traditional cellular network is formed by a regular deployment of the base stations in a hexagonal pattern according to the cellular concept across the network coverage area. In Chapter 3, a coordinated resource allocation framework is proposed for traditional cellular networks. The resource allocation in the proposed framework is both adaptive and localized. The frequency resources assigned to the BSs adapt to the varying traffic 
distribution across the network. This is in contrast with the current resource allocation frameworks where the resources assigned to the BSs are more or less static with limited adaptation. The performance results of the proposed framework reveal significant improvements compared to the current resource allocation strategies. The work of this Chapter has been partly published in $[13,14]$.

In the first part of Chapter 4 a network shadow fading model has been proposed. To our knowledge, the literature has not reported on the correlation between the channel gains of pairs of links with no common end. This is due to the fact that the models in the literature have been proposed for the traditional cellular network. In these networks, it is reasonably assumed that the channel gains of pairs of links between terminals and two different BSs are not correlated due to the sufficiently large distances between the BSs. In autonomous cellular networks, on the other hand, it is expected that a large number of BSs will be deployed in random positions. The irregularity of the BS deployment can result in dense clusters within the same locality, i.e. with similar/correlated topographical properties. In this case, the correlation between a pair of links between two terminals and two base stations becomes crucial for a realistic evaluation of the system performance. The work of this part has been published in [15].

In the literature, the resource allocation frameworks for cellular networks have been developed based on the assumption of a regular deployment of the BSs. In a cellular network with an irregular deployment of the BSs, these frameworks are not applicable. In the second part of Chapter 4, we have developed a unified resource allocation framework which can be applied to any cellular network regardless of the BS deployment pattern. In order to do so, a network clustering algorithm is proposed which essentially clusters the BSs in the network to clusters of coordinating BSs. A coordinated resource allocation strategy, first proposed in [33], is tailored according to our assumptions, requirements and objectives and incorporated in the resource allocation framework. The performance evaluations show desirable results. The work of this part has been partly published 
in $[16]$.

\subsection{Scope}

- Multiple-antenna technologies such as beam-forming, spatial multiplexing, spacetime coding, etc. have not been considered.

- A delay-tolerant infinitely back-logged data traffic model with no specific Quality of Service requirements has been considered. The focus of this work is on the design of the infrastructure, hence a simple traffic model is assumed to avoid any traffic-related issues.

- The proposed resource allocation frameworks are applicable to any Orthogonal Frequency Division Multiple Access (OFDMA) system. However, the LTE standard has been adopted for performance evaluation.

- The performance evaluation is exclusively conducted for down-link communication. This is partly due to the fact that the down-link communication is often considered as the bottleneck in the cellular networks.

\subsection{Outline}

- Chapter 2 begins with a rigorous mathematical model for a cellular network operating on multiple frequency channels in Section 2.1. Subsequently, well-known frequency reuse strategies are reviewed in Section 2.2. A literature review on coordinated resource allocation in cellular networks is provided in Section 2.3. Proportional fairness is studied in Section 2.4 and finally practical considerations in LTE systems together with the standard performance metrics for a cellular network are reviewed in Section 2.5. 
- Chapter 3 presents a coordinated resource allocation framework for the traditional cellular network. In Section 3.1 the concept of the scheduling cell is introduced. Terminal grouping is discussed in Section 3.3.1. Subsequently, the coordinated resource allocation strategy is developed in Section 3.3.

- Chapter 4 introduces the autonomous cellular network. In Section 4.1, a network shadow fading model is proposed. In the second part an adaptive resource allocation framework for autonomous cellular networks is developed. The network clustering method is presented in Sections 4.2.1, 4.2.2 and 4.2.3. The coordinated resource allocation framework is developed in Section 4.2.4. The performance of the proposed framework is evaluated in Section 4.4.

- Chapter 5 concludes the dissertation with a summary of the work and possible future research directions. 


\section{Chapter 2}

\section{Literature review and background material}

\subsection{Cellular network: A definition}

In order to provide service to a set of $K$ wireless terminals, a set of $A$ base stations (BS) are deployed. Each BS a has a backbone connection to the wired network and a transmission power budget of $P_{a}$. A traditional cellular network is a special case of this model, where all BSs have the same power budget and are deployed according to a regular pattern modeled with hexagons [28].

Each terminal communicates with only one BS. Terminal $k_{l}$ is assigned to BS $a(l)$ if the large-scale fading channel gain between terminal $k_{l}$ and $\mathrm{BS} a(l)$ is the strongest among all BSs. Consequently BS $a_{i}$ will serve the set of terminals $\mathcal{K}_{i}=\left\{k_{l} \mid a(l)=a_{i}\right\}$ such that $\sum_{i=1}^{A}\left|\mathcal{K}_{i}\right|=K$. The wireless communication between the BSs and the terminals are established on the available frequency spectrum, a set of $N$ (frequency) resources. Most cellular networks are time-slotted which means the resources are allocated once every time slot - also known as a Transmission Time Interval (TTI).

Unless otherwise stated, it is assumed that the power budget $P_{i}$ is uniformly dis- 
tributed across the available sub-carriers. Assuming each frequency resource is comprised of $M$ sub-carriers, the power budget for transmission on one sub-carrier at BS $a_{i}$ is:

$$
p_{i}=\frac{P_{i}}{M N}
$$

In a network with one $\mathrm{BS}$ and $K$ terminals, the resource allocation problem can be formulated as follows: In a given time slot $t$, matrix $\mathbf{I}(t) \in\{0,1\}^{K \times N}$ determines the frequency channel allocations: $I_{l n}(t)=1$ if frequency channel $n$ is assigned to terminal $k_{l}$ and $I_{l n}(t)=0$ otherwise. Based on the allocation matrix $\mathbf{I}(t)$, the data rate of terminal $k_{l}$ in time slot $t$ is:

$$
r_{l}(t)=\sum_{n=1}^{N} r_{l n}(t) I_{l n}(t)
$$

where, $r_{l n}(t)$ is the supported data rate on frequency channel $n$ by terminal $k_{l}$ in time slot $t$.

Let $U_{l}\left(r_{l}(t)\right)$ be the utility function of terminal $k_{l}$ in time slot $t$. The resource allocation problem at the beginning of time slot $t$ can be formulated as [32]:

$$
\begin{aligned}
& \max _{\mathbf{I}(t)} \sum_{l=1}^{K} U_{l}\left(r_{l}(t)\right) \\
& \text { subject to }\left\{\begin{array}{l}
\sum_{l=1}^{K} I_{l n}(t)=1 \text { for } n=1,2, \cdots, N \\
I_{l n}(t) \in\{0,1\} \quad \text { for } \quad l=1,2, \cdots, K \quad, \quad n=1,2, \cdots, N
\end{array}\right.
\end{aligned}
$$

It is assumed that each BS is equipped with one antenna. Hence, allocating the same resource to two independent simultaneous transmissions from the same BS is not allowed as it would create excessive interference levels [19]. Consequently, the first constraint in (2.1) guarantees that each resource is allocated to exactly one transmission per TTI. The second constraint dismisses the option of time-sharing during a TTI.

In a cellular network with $A$ BSs, the scheduling problem can be generalized as: 


$$
\begin{aligned}
& \max _{\left\{\mathbf{I}_{1}(t), \cdots, \mathbf{I}_{A}(t)\right\}} \sum_{l=1}^{K} U_{l}\left(r_{l}(t)\right) \\
& \text { subject to } \begin{cases}\sum_{l \in\left\{l \mid k_{l} \in \mathcal{K}_{a}\right\}} I_{l n}(t) \leq 1 & \text { for } \quad n=1, \cdots, N, a=1, \cdots, A \\
I_{l n}(t) \in\{0,1\} & \text { for } \quad l=1, \cdots, K, j=1, \cdots, N\end{cases}
\end{aligned}
$$

where $\mathbf{I}_{a}(t)$ is the frequency allocation matrix for BS $a$.

A cellular network which allocates the resources based on (2.2) is referred to as a fully-coordinated network. It should be noted that full coordination among all BSs in the network is extremely costly as it involves joint resource allocation of all communication links in the network.

In the literature, a cellular network is referred to a network with a set of BSs which are regularly deployed according to a hexagonal pattern. In the model presented in this Section, however, there is no assumption on the BS deployment pattern. We refer to a network with regularly deployed BSs as a traditional cellular network. In Section 2.2, we study how the resource allocation problem (2.2) has been tackled in the past couple of decades. The recent advances in coordinated resource allocation of traditional cellular networks are studied in Section 2.3. In Chapter 3, a localized and adaptive resource allocation framework with a degree of coordination between BSs is proposed assuming a regular deployment pattern for the BSs. The performance is evaluated and compared against the well-known resource allocation methods discussed in Section 2.2. In Chapter 4 , there is no assumption on the BS deployment pattern. The BSs are deployed in an unknown irregular pattern. A coordinated resource allocation framework is proposed. Throughout the thesis, the goal of resource allocation is to maintain proportional fairness among the active terminals in the network. This concept is extensively discussed in Section 2.4. Section 2.5 concludes the literature review with some practical considerations in the design and the performance evaluation of cellular networks. 


\section{$2.2 \quad$ Frequency reuse strategies}

The fully coordinated resource allocation problem formulated in (2.2) is prohibitively complex. It inevitably should be broken down into smaller sub-problems. In Section 2.2.1 we study how this problem is tackled in traditional networks. Subsequently in Section 2.2.2, a more recent and more aggressive approach to frequency reuse, namely the fractional frequency reuse (FFR), is studied.

\subsubsection{The traditional approach}

Proposed in the late 1970's, the cellular concept is one of the most important breakthroughs in wireless communication over the past few decades. In earlier mobile radio systems a large coverage area would be served by one high powered transmitter and the maximum number of simultaneous calls was very limited (e.g. maximum of twelve calls in an area of a thousand square miles) [30]. In a cellular network, the same coverage area is served by many (instead of one) BSs. Every $F$ neighboring BSs form a so-called frequency reuse cluster within which area no reuse of the spectrum is allowed. The available frequency spectrum, $\mathcal{N}$, is partitioned into $F$ subsets $\left\{\mathcal{N}_{1}, \mathcal{N}_{2}, \cdots, \mathcal{N}_{F}\right\}$ with $\left|\mathcal{N}_{1}\right|=\left|\mathcal{N}_{2}\right|=\cdots=\left|\mathcal{N}_{F}\right|$. Each subset denotes the available spectrum to a particular BS. The frequency assignments to the BSs is according to a static frequency reuse pattern which allows a continuous reuse of the frequency resources across the network. The parameter $F$ is referred to as the frequency re-use factor (FRF) which determines the size of the clusters and in a sense the level of frequency reuse in the network. In Figure 2.1 the frequency reuse pattern in a cellular network with $F=3$ is illustrated. Each cell is depicted as a hexagon with the base station located at the center. The subset in each hexagon denotes the frequency allocation for that particular cell.

With a fixed number of frequency resources allocated to each BS, the resource allocation problem in (2.2) is significantly simplified. In order to avoid excessive interference, 


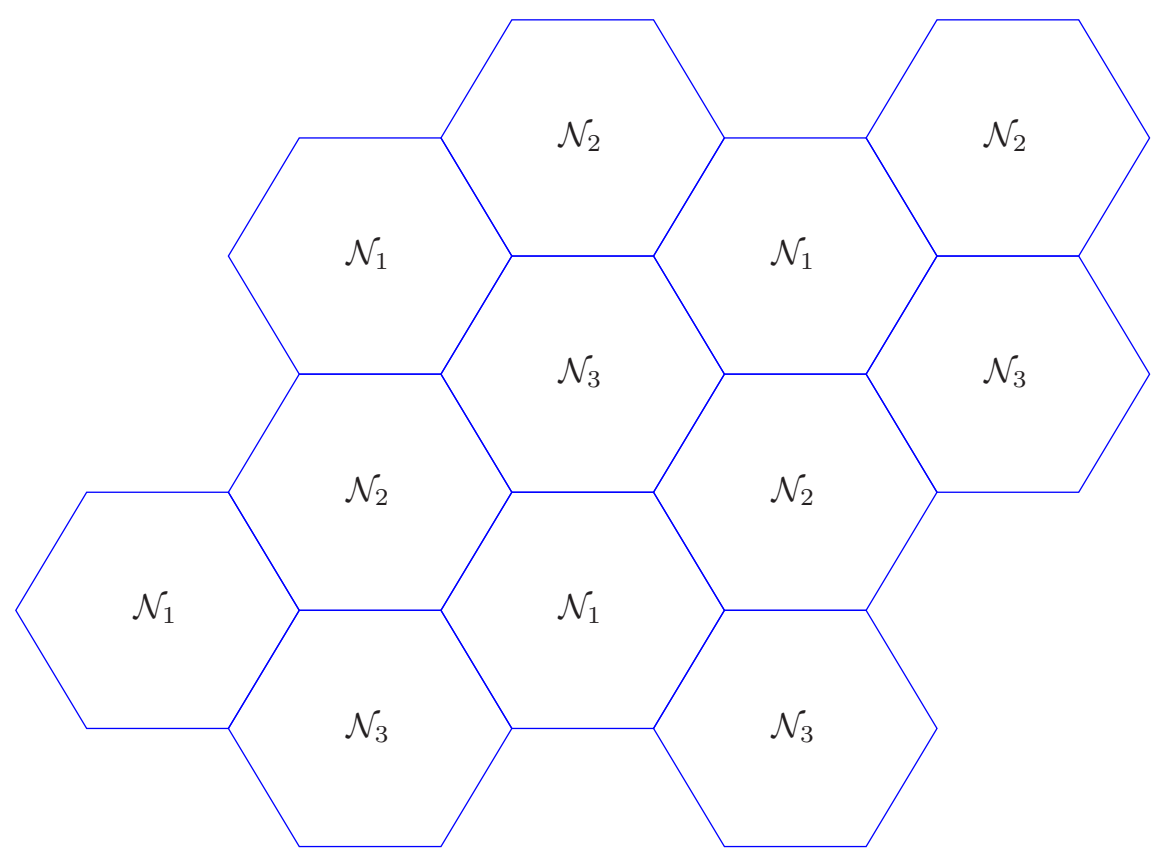

Figure 2.1: Traditional cellular network with $F=3$

high frequency reuse factors were employed in the earlier cellular networks. However, due to the tremendously growing need for more capacity, more aggressive frequency reuse schemes are required. In the next section, we study a recently proposed and widely investigated frequency reuse strategy.

\subsubsection{Fractional frequency reuse}

In order to satisfy the high transmission rates in modern cellular networks, an aggressive frequency reuse is indispensable. In the model proposed by the original cellular concept, the most aggressive frequency reuse is achieved by setting $F$ equal to unity, which is often referred to as the universal frequency reuse. In this case, the entire spectrum, $\mathcal{N}$, is available to all BSs. In other words, the largest possible pool of frequency resources is allocated to each cell. However, a group of terminals - often located at the edge of the cell with weak channel gains to the BS - suffer from the excessive interference from the neighboring cells. The main idea behind fractional frequency reuse is to protect this 
group of weak terminals while maintaining a frequency reuse factor close to unity. In order to do so, the terminals in each cell are partitioned into two groups. Two different frequency reuse patterns are applied: A conservative frequency reuse is employed for the weak terminals while a more aggressive reuse pattern is applied for the stronger terminals, which are generally located closer to the $\mathrm{BS}^{1}$.

Let us assume the cell coverage area is divided into three sectors, $S_{1}, S_{2}$ and $S_{3}$ as depicted in Figure 2.2. The BS is assumed to be located at the center of the cell with three fixed $120^{\circ}$ beams toward the sectors. The terminals are partitioned into the strong and weak terminal sets based on their distances (or SNR levels) to the BS. Terminals with higher distances (or lower SNR levels) than a certain threshold form the weak terminal set. In Figure 2.2, the boundary between the two groups is shown by the dotted circle at the center of the cell. The same sectorization pattern and terminal grouping is applied to all cells in the network.

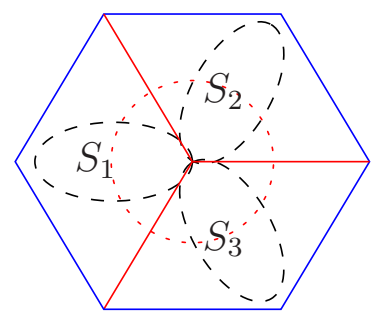

Figure 2.2: Cell coverage area

The weak terminal set are served with a higher transmission power. In addition, in order to avoid excessive interference from the neighboring BSs to the weak terminal set, the transmission across the three sectors are orthogonal. Depending on how the frequency resources and power budgets are allocated to the defined regions, two variations of the FFR technique have been proposed.

\footnotetext{
${ }^{1}$ The idea of employing multiple frequency reuse patterns in a cellular network dates back to the early 1980 s and was first proposed in [18].
} 


\section{Soft Frequency Reuse (SFR)}

Let us assume that the BS has a total power budget of $3 P_{0}$ with a total available spectrum of $W$. With an equal distribution of the power budget across the three sectors, each sector will have a power budget of $P_{0}$. The SFR strategy, divides the spectrum into three equal parts of $W / 3$. The terminals are served at two power levels: the cell edge terminals are served over one-third of the spectrum at a higher transmit power and the cell center terminals are served over the remaining two-thirds of the spectrum at a lower transmit power. The power-frequency allocation for the three sectors is depicted in Figure 2.3. The values in this figure are normalized by $\frac{P_{0}}{W}$ such that the total transmit power in each sector equals unity. In region $S_{1}$, the cell edge terminals are served over the shaded one-third of the spectrum with a normalized PSD of $\alpha$. The center region is served over the remaining two-thirds of the spectrum at a lower transmission power with normalized PSD of $\frac{(3-\alpha)}{2}$. Similar frequency reuse patterns are proposed for regions $S_{2}$ and $S_{3}$. Note that the three cell edge regions are served on orthogonal frequency resources in order to reduce the interference on these links.

\section{Partial Frequency Reuse (PFR)}

In this approach, a more conservative reuse strategy is proposed. Unlike SFR, the spectrum allocated to the cell edge terminals in one region is not reused at the center of the other two regions in the cell. Two variations of this method are depicted in Figure 2.4. In the approach on the left-hand side, equal power levels are allocated to the cell center and cell edge regions. The right-hand strategy amplifies the transmission power to the cell edge region by a factor of 3 . The parameters $\beta$ and $\gamma$ should be carefully chosen to satisfy the power budget constraints of each BS. 


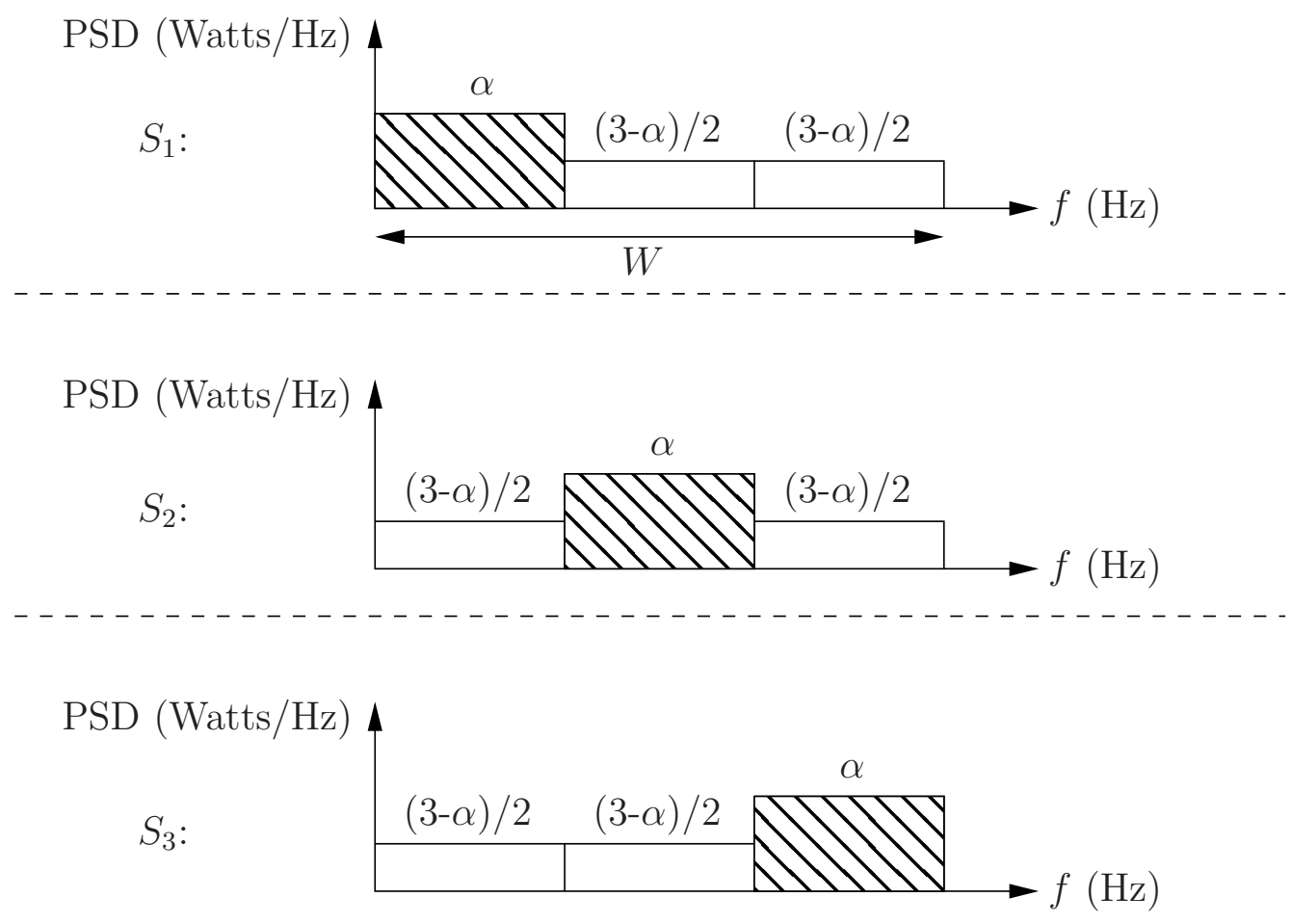

Figure 2.3: Soft frequency reuse
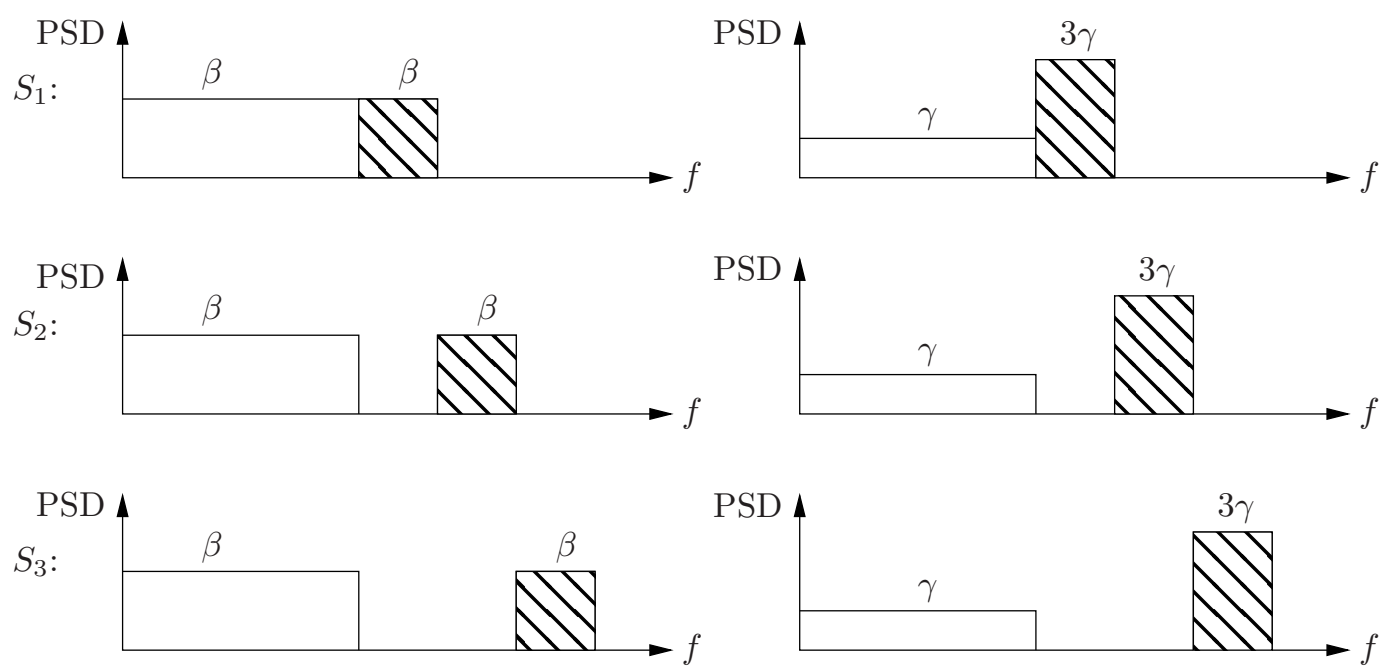

Figure 2.4: Partial frequency reuse 


\subsection{Coordinated adaptive resource allocation}

Based on the cellular concept, a fixed system-wide frequency reuse strategy reduces the inter-cell-interference (ICI) to tolerable levels in a simple and straightforward manner. The in-cell capacity is then optimized by the design of an appropriate air interface technology. This is achieved at the cost of a significant loss of efficiency in using the available spectrum. This is due to the fact that different geographical locations do not have similar traffic distributions and call for different frequency reuse patterns. This in turn becomes extremely important for smaller cells where each BS carries less traffic and as a result more abrupt variations in the traffic distribution across the network are possible. The coordination of resource allocation between neighboring BSs has emerged as a potential solution for a more adaptive method for frequency reuse ${ }^{2}$. In this section, a literature review on two major topics in the area is provided.

\subsubsection{Two-level resource allocation}

Bonald et al. introduce coordination between cells as a new notion in scheduling for a TDMA cellular network [7]. The idea of resource allocation in two different time-scales is presented. In the first phase, the activity of interfering BSs is coordinated in order to maximize the transmission data rates. In the second phase, load balancing diverts traffic from heavily-loaded cells to lightly-loaded cells. The paper focuses on the first phase and proposes an interference avoidance model. It is assumed that BS $a$ is either active - in which case transmits at maximum power to a single terminal - or turned off. The transmission profile $j$ is defined as the set of $\mathrm{BSs} \mathcal{A}_{j} \subset \mathcal{A}$ which are active. A set of transmission profiles $\left\{\mathcal{A}_{1}, \cdots, \mathcal{A}_{J}\right\}$ are formed. The interference avoidance strategy is to choose the appropriate transmission profile in order to maximize the transmission rates across the network. A similar technique is proposed in [31] for TDMA cellular networks. Two states are considered for each BS: either the BS transmits at maximum power or the

\footnotetext{
${ }^{2}$ The coordination between BSs is often referred to as inter-cell interference coordination (ICIC).
} 
BS is off. Based on this, different frequency reuse patterns (similar to the transmission profiles in [7]) are defined according to the ON/OFF activities of the BSs in the network. The first phase of resource allocation decides on the reuse pattern to be used and in the second phase all active BSs independently assign the frequency channel to a terminal in their corresponding cell according to the reuse pattern prescribed by the first phase.

Li and Liu extend the two-level resource allocation framework to an OFDMA system [26]. A radio network controller assigns the available spectrum to all terminals in the network in a centralized fashion during the first phase. More specifically, through an exhaustive search over all BSs and terminals the frequency channels are assigned to the links with the goal of maximizing the sum throughput. Due to the bursty nature of the traffic, the allocated terminals might not have any traffic to be communicated. Hence in the second phase, each base station modifies the channel assignments within the cell based on the amount of available traffic for each terminal.

Rahman and Yanikomeroglu propose a two-level resource allocation strategy in [29]. A group of neighboring BSs are connected to a central controller. In the first phase, the central controller assigns the frequency resources to the BSs based on the current traffic in each cell. In the second phase, each BS performs terminal scheduling independently based on the frequency assignments of the first phase.

When studying the resource allocation in cellular networks, one major issue is often neglected. Namely, no prior information is available about the out-of-cell interference at the time of allocation of resources in each time slot. In [11], the authors propose a twolevel resource allocation framework which takes this lack of information into account. In the first phase, an interference graph based on the geographical location of the terminals is formed. Based on this graph, the set of co-channel terminals in the network is formed. In the second phase, the available frequency channels are assigned to the co-channel terminal sets formed in the first phase. For terminal scheduling in the second phase, only the signal-to-noise ratios of the terminals in each cell are considered. 
All two-level resource allocation schemes are proposed based on a common principle: The first phase decides on an appropriate frequency reuse pattern based on the slowvarying traffic distribution of the cells. At a faster time-scale, the second phase fine tunes the resource allocation in each cell independently based on the fast variations in the traffic patterns.

\subsubsection{Adaptive FFR techniques}

The FFR strategy discussed in Section 2.2.2 has two major shortcomings: First, the terminals are partitioned into the weak and strong groups solely based on their distances from their assigned BSs. The interfering BSs are not considered. Second, the frequency resources assigned to the defined regions do not adapt to the variations in the network traffic. In this section, we discuss two adaptive FFR techniques which introduce some degree of adaptation to the traffic distributions and radio channel characteristics.

In [9], an adaptive FFR is presented as one interference coordination measure in the 4G networks. Three frequency-power profiles are introduced: The first corresponding to FRF of 1, the second corresponding to FRF of 3 and the last corresponds to the PFR method discussed in Section 2.2.2 (see Figure 2.4). The proposed adaptive scheme switches between the three profiles based on the distribution of traffic in each cell.

Ali and Leung propose a more elaborate dynamic frequency allocation technique in [3]. Unlike static FFR, the terminals are not statically partitioned into two sets. In other words, all terminals are potentially a member of both groups. It is the BS which decides a terminal belongs to which group at each time slot. With the general model of the FFR reuse in mind, a two-level resource allocation approach is presented. In the first phase, a centralized radio network controller assigns the frequency resources to the defined regions in each cell (see Figure 2.2). The assignment is performed based on the average performance of all terminals in the network on all available frequency

resources. The frequency reuse pattern is then applied to all cells in the network. In 
the second phase, the BSs independently assign the frequency resources to the terminals in accordance with the frequency assignment decisions of the radio network controller in the first phase. The frequency assignment decisions at this level are based on a minimum performance guarantee scheduling method.

\subsection{Resource allocation strategies and trade-offs}

In a cellular network, the available resources are the power budget and the frequency spectrum. The resource assignments can be studied from two perspectives:

- Subscriber: The service of a subscriber is usually defined by the Quality of Service (QoS) requirements, e.g. minimum data rate, maximum delay, maximum packet drop probability, etc. With no specific QoS requirement, the service can be defined by the data rate provided to each subscriber. Hence each subscriber demands the highest possible supported data rate.

- Network: From the network point of view, in order to maximize the sum throughput, each resource should be allocated to the terminal with the strongest channel gain. Despite the high achieved bit rates, all except a few subscribers will be deprived of any service. This is an immediate result of the inevitable disparities in the channel gains due to the random locations of subscribers in the network.

In order to achieve a reasonable compromise between the two viewpoints, we here re-visit a concept in Economics: In any system, there exist a set of resources and a set of entities (e.g. machines) with certain demands. The assignment of a system resource for a service to an entity, brings a benefit (utility) to that entity and imposes a cost

on the system. The marginal utility of a service is the utility gained (or lost) from an increase (or decrease) of that service. It suggests that there exist diminishing returns in consumption, so that the first unit of consumption of a service yields more utility than the second and subsequent units. 
Based on the law of diminishing returns, the marginal utility decreases as the relative (proportional) improvement in the data rate decreases. In other words, increasing the data rate of a user with already a $1 \mathrm{Mbps}$ communication link by $10 \mathrm{Kbps}$ does not result in the same utility as increasing the data rate of a user with a 50Kbps link by the same amount. The logarithm function abides by the rule of diminishing marginal utility. Hence, we define the utility of each terminal by not the data rate but the logarithm of the supported data rate. Thus the network designer develops the resource assignment methods to maximize the sum logarithm of the throughputs which achieves a reasonable balance between the sum throughput and the individual terminal data rates. Such strategy is ofter referred to as proportional fair (PF) scheduling. The PF scheduler was first introduced for a CDMA/HDR cellular network [6]. Over the past decade, this scheduler has gained a lot of popularity in cellular networks. In the following section, the properties of the PF scheduler together with some recent advances and challenges are investigated.

\subsubsection{Proportional fairness}

Definition (Proportional fairness) An allocation of rates $\mathbf{r}^{*}=\left[r_{1}^{*}, r_{2}^{*}, \cdots, r_{K}^{*}\right]^{T}$ is proportionally fair if and only if, for any other feasible allocation $\mathbf{r}$, we have:

$$
\sum_{k=1}^{K} \frac{r_{k}-r_{k}^{*}}{r_{k}^{*}} \leq 0
$$

Let $r_{k}=a_{k} r_{k}^{*}$ be a feasible rate allocation. It is easy to show that

$$
\frac{1}{K} \sum_{k=1}^{K} a_{k} \leq 1
$$

Since the geometric mean is always less or equal to the arithmetic mean, we have

$$
\prod_{k=1}^{K} a_{k} \leq 1
$$


or,

$$
r_{1} r_{2} \cdots r_{K} \leq r_{1}^{*} r_{2}^{*} \cdots r_{K}^{*}
$$

which results in the well-known optimization problem for PF scheduling [8, sec. 1.2.2], namely,

$$
\max _{\mathbf{r}} \sum_{k} \log r_{k}
$$

Based on (2.4), it is often said that increasing one user's rate by $x \%$ in a proportional fair allocation would cause a cumulative percentage decrease of more than $x \%$ in other users' rates.

Let us assume a single BS and $K$ users in a TDMA system. Let $p_{k}$ be the access probability of user $k$ to the channel and let $r_{k}$ denote the supported rate of user $k$ once it has been granted access. For simplicity we assume the rate $r_{k}$ to be static for all time slots. The sum-log maximization problem can be written as:

$$
\begin{aligned}
& \max _{\mathbf{p}} \sum_{k=1}^{K} \log \left(p_{k} r_{k}\right) \\
& \text { subject to } \sum_{k=1}^{K} p_{k}=1 \quad, \quad p_{k} \geq 0
\end{aligned}
$$

Reformulating the optimization problem in the standard form, we have:

$$
\begin{aligned}
& \min _{\mathbf{p}}-\sum_{k=1}^{K} \log \left(p_{k} r_{k}\right) \\
& \text { subject to }\left\{\begin{array}{l}
\sum_{k=1}^{K} p_{k}-1=0 \\
-p_{k} \leq 0 \text { for } k=1, \cdots, K
\end{array}\right.
\end{aligned}
$$


Writing the KKT conditions we have:

$$
\left.\begin{array}{l}
-\frac{1}{p_{k}}-\lambda_{k}+\nu=0 \\
\lambda_{k} p_{k}=0
\end{array}\right\} \Rightarrow p_{k}=\frac{1}{\nu}, \forall k
$$

which means in a TDMA system, the PF scheduler achieves equal access time for all terminals in the network.

The key idea in PF scheduling is to discard the channel disparities by averaging out channel variations due to path loss and shadow fading. The scheduler then exploits multi-user diversity gains on the normalized channel conditions. It should be noted that the higher the disparities in the channel gains of the subscriber population, the lower the sum throughput of a PF scheduler. This notion has been closely studied in [36]. The authors argue that the loss in sum throughput of a PF scheduler might not be acceptable and propose a two stage scheduler. On one level, PF scheduling is applied to groups of subscribers with acceptably low channel gain disparities. The second stage applies the sum throughput maximizing scheduler over the groups of the previous stage (which comprise the entire subscriber population). In [24], another approach to resolving the sum capacity-fairness trade-off is proposed. With introducing a control parameter, the generalized PF scheduler is capable of putting more or less weight on sum maximization for scheduling decisions in each time slot. Provided the current QoS level of all users in the network is above the required level (i.e. QoS margin is positive for all users), emphasizing the effect of sum capacity maximization on scheduling decisions will increase the sum throughput of the system without violating any QoS requirements. In other words, an appropriate choice of the scheduler's control parameter can turn a current surplus in the QoS levels of the users to a higher sum capacity of the system. User-specific control parameters are defined in [5] and an adaptive PF (APF) scheduler is proposed. The authors take the ratio of allocated data rate and the mean channel data rate of each user as the measure of fairness. In this way, a scheduler is deemed to be fair if these ratios 
are the same for all active terminals in the system.

PF resource scheduling has two drawbacks from a practical standpoint:

- If the channel variations are too fast, channel estimates will not be accurate enough for PF scheduling. However, if the channel variations are not fast enough, it will take a long time for a user to access the system resources again and fairness in a desired time frame cannot be established - it should be noted that the classical PF scheduler achieves proportional fairness over a considerably large time frame which sometimes may not be practical.

- If the number of users is very large, the average access time of each user will decrease accordingly. However, the granularity of the access time of a given system is fixed and equal to the duration of a time slot. Consequently, the system might not be able to accommodate the PF scheduler.

Based on these two observations the authors of [35], study the Average Access Time (AAT), Average Access Rate (AAR) and Average Waiting Time (AWT) in a multi-user system with a single BS. Although the original PF scheduler maximizes the ratio between the instantaneous and average rates, this paper considers the ratio between the average and instantaneous SINRs to make the scheduling decisions. It is mathematically shown that this scheduler indeed achieves equal AAT for all users. This is quite intuitive as once the channel gain disparities are discarded (by normalizing either the rate or SINR) each user is equally likely to access the system resources which results in equal AAT for all.

In multi-carrier systems, e.g. OFDMA, with $N$ orthogonal frequency channels the resource allocation problem becomes more complex. In [23], it is shown that in order to achieve proportional fairness in a multi-carrier system the following optimization problem should be solved: 


$$
\max \prod_{k}\left(1+\frac{\sum_{n \in \mathcal{N}_{k}} r_{k, n}(t)}{(T-1) \bar{r}_{k}(t)}\right)
$$

where $\mathcal{N}_{k}$ is the frequency resources assigned to user $k$ in the current time slot. And $T$ is the averaging window. The average rate of terminal $k$ at time slot $t$ is

$$
\bar{r}_{k}(t)=\left(1-\frac{1}{T}\right) \bar{r}_{k}(t-1)+\frac{1}{T} r_{k}(t)
$$

A larger parameter $T$ would result in a longer time average rate.

For the special case of TDMA systems (i.e. $|\mathcal{N}|=1$ ), the PF scheduling simplifies to:

$$
k^{*}=\operatorname{argmax}_{k} \frac{r_{k}(t)}{\bar{r}_{k}(t)}
$$

where $k^{*}$ is the terminal to be scheduled in time slot $t$.

In [4], three heuristic strategies for multi-carrier systems have been proposed:

- Scheme 1: Independent parallel scheduling on each frequency channel

1. For $n=1$ to $N$

2. $\quad k_{n}^{*}=\operatorname{argmax}_{k} \frac{r_{k, n}}{\bar{r}_{k, n}}$

3. End

- Scheme 2: Ordered resource allocation with partial updating

1. For $n=1$ to $N$

2. $\quad k_{n}^{*}=\operatorname{argmax}_{k} \frac{r_{k, n}}{\bar{r}_{k}}$

3. $\quad \bar{r}_{k}=\left(1-\frac{1}{T}\right) \bar{r}_{k}+\frac{1}{T} r_{k, n}$

4. End 
- Scheme 3: Resource allocation with no partial updating

1. For $n=1$ to $N$

2. $\quad k_{n}^{*}=\operatorname{argmax}_{k} \frac{r_{k, n}}{\bar{r}_{n}}$

3. End

It should be noted that with partial updating (scheme 2) the order of allocating the frequency channels becomes important. In [20], a heuristic, independent of the allocation order, is proposed.

\subsection{Practical considerations}

Throughout the thesis, the long term evolution (LTE) air interface technology has been considered and the numerical results are presented based on system-level simulations of the cellular network. In Section 2.5.1 the available frequency-time resources in this standard are reviewed. A link-level performance model is discussed in Section 2.5.3. Finally, the two performance metrics which are widely used in the performance evaluation of the cellular networks are introduced in Section 2.5.4.

\subsubsection{Scheduling granularity}

For practical considerations, the smallest granularity for scheduling in all OFDMA standards, including LTE, is defined to be larger than one sub-carrier wide (in frequency) and more than one OFDM symbol long (in time). The following terms define the frequencytime resources in the LTE standard [22]:

- Resource element is the smallest time-frequency resource in the system - a block of 1 OFDM symbol long and 1 sub-carrier wide. The duration of each OFDM symbol 
is $\frac{1}{14} \mathrm{~ms}$ (including the cyclic prefix) and each sub-carrier occupies $15 \mathrm{KHz}$ of the spectrum.

- Physical resource block $(P R B)$ is a $180 \mathrm{KHz} \times 0.5 \mathrm{~ms}$ block of frequency-time resource, which essentially forms a block of $12 \times 7$ resource elements.

- Sub-frame is defined as a 1ms block in time equivalent to the length of 14 OFDM symbols, which is subdivided into two $0.5 \mathrm{~ms}$ slots. A sub-frame is also referred to as the transmission time interval (TTI) of the system.

- The scheduling in LTE is performed every sub-frame and the smallest resource allocation granularity is a pair of consecutive PRBs (in time) which amounts to a block of 12 sub-carriers wide and 14 OFDM symbols long. This block is often referred to as a scheduling block (SB) which is $180 \mathrm{KHz}$ wide and $1 \mathrm{~ms}$ long (see Figure 2.5).

\subsubsection{Channel model}

The computer simulations are performed in accordance with the International Telecommunications Union (ITU) recommendations report M-2135 [2]. Based on this report, the wireless channel is composed of $N_{c}$ paths, here referred to as clusters. Each cluster $n=1,2, \cdots, N_{c}$ has a corresponding delay of $\tau_{n}$. The channel impulse response between transmit antenna $s$ and receive antenna $d$ is

$$
h_{d s}(t)=\sum_{n=1}^{N_{c}} g_{d s}(t, n) \delta\left(t-\tau_{n}\right)
$$

where, $g_{d s}(t, n)$ is the channel gain of cluster $n$ between transmit antenna $s$ and receive antenna $d$.

The root mean square (RMS) delay spread $\tau_{\text {RMS }}$ is modeled as a log-normal random variable with mean $\mu_{\tau}$ and standard variation $\sigma_{\tau}$. The random delays of the clusters are 


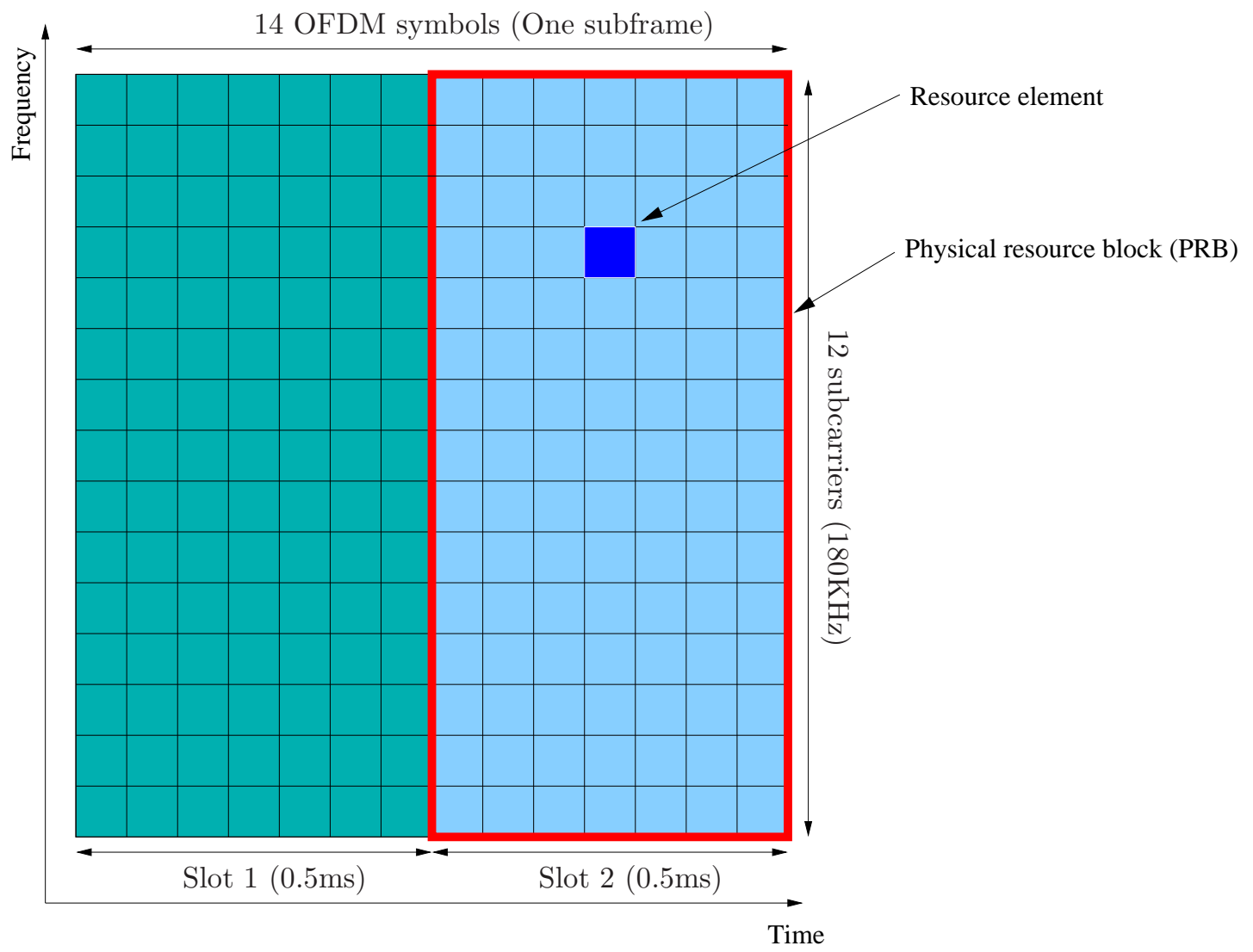

Figure 2.5: A scheduling block 
exponentially distributed and are generated as follows:

$$
\tau_{n}^{\prime}=-a_{\tau} \tau_{\mathrm{RMS}} \ln x
$$

where $a_{\tau}$ is a scaling factor based on measurements which takes the statistical relationship between path delays and powers into account. $x$ is a uniformly distributed random variable between 0 and 1 . The actual delays are derived after the following adjustment:

$$
\tau_{n}=\operatorname{sort}\left(\tau_{n}^{\prime}-\min _{m} \tau_{m}^{\prime}\right)
$$

The power of cluster $n$ follows an exponential power delay profile and is calculated as follows:

$$
P_{n}^{\prime}=\exp \left(-\tau_{n} \frac{a_{\tau}-1}{a_{\tau} \sigma_{\tau}}\right) 10^{Z_{n} / 10}
$$

where, $Z_{n} \sim \mathcal{N}\left(0, \mu_{s}\right)$ is the per-cluster shadowing term in the logarithmic scale. The actual cluster powers are calculated based on the following normalization ${ }^{3}$ :

$$
P_{n}=\frac{P_{n}^{\prime}}{\sum_{n=1}^{N} P_{n}^{\prime}}
$$

Each cluster is defined by the summing contribution of $N_{r}$ rays (plane waves) with the same randomly generated power level $\left(\frac{P_{n}}{N_{r}}\right)$, delay $\left(\tau_{n}\right)$ and average angle of arrival/departure (AoA/AoD).

The average angle of departure for cluster $n$ is generated as follows. The RMS angle of departure is modeled as a log-normal random variable with mean $\mu_{\phi}$ and standard deviation $\sigma_{\phi}$. The average angle of departure is then generated as:

$$
\bar{\phi}_{n}^{\prime}=\frac{2 \sigma_{\phi}}{C} \sqrt{-\ln \left(\frac{P_{n}}{\max P_{n}}\right)}
$$

\footnotetext{
${ }^{3}$ The total power will be scaled according to the path-loss and shadow fading channel gains.
} 
where, $C$ is a constant for the calculation of angle of departure. The actual angle of departure is derived as follows:

$$
\bar{\phi}_{n}=X_{n} \bar{\phi}_{n}^{\prime}+Y_{n}+\phi_{\mathrm{LOS}}
$$

where, $X_{n}$ is a discrete random variable uniformly distributed in the set $\{-1,1\}, Y_{n} \sim$ $\mathcal{N}\left(0, \frac{\sigma_{\phi}}{7}\right)$ to introduce variations and $\phi_{\mathrm{LOS}}$ is the relative direction between the transmitter and receiver. Finally, the ray angles of departure are calculated by offsetting the corresponding average angles with a constant $N_{r} \times 1$ offsetting vector provided in Table 2.1 .

$$
\phi_{n, m}=\bar{\phi}_{n}+c_{d} \alpha_{m}
$$

where $c_{d}$ is the cluster-wise RMS azimuth spread of the departure angles. The generation of angles of arrivals follows a similar procedure. For the channel parameters in different environments and more information on the channel modeling, the reader is referred to the ITU-2135 report [2].

Table 2.1: Ray offset angles within a cluster for a normalized RMS angle spread

\begin{tabular}{cc}
\hline Ray number & Basis vector of offset angles $\left(\alpha_{m}\right)$ \\
\hline 1,2 & \pm 0.0447 \\
3,4 & \pm 0.1413 \\
5,6 & \pm 0.2492 \\
7,8 & \pm 0.3715 \\
9,10 & \pm 0.5129 \\
11,12 & \pm 0.6797 \\
13,14 & \pm 0.8844 \\
15,16 & \pm 1.1481 \\
17,18 & \pm 1.5195 \\
19,20 & \pm 2.1551 \\
\hline
\end{tabular}

Antenna polarization is not considered and an omni-directional field pattern for all antenna elements is assumed. The channel gain of cluster $n$ between transmit antenna $s$ 
and receive antenna $d$ can be written as

$$
\begin{aligned}
g_{d s}(t, n)=\sqrt{\frac{P_{n}}{N_{r}}} \sum_{m=1}^{N_{r}} \exp \left(j d_{s} \frac{2 \pi}{\lambda} \sin \left(\phi_{n, m}\right)\right) \\
\quad \exp \left(j d_{d} \frac{2 \pi}{\lambda} \sin \left(\varphi_{n, m}\right)\right) \exp \left(j \psi_{n, m}\right) \exp \left(j 2 \pi f_{n, m} t\right)
\end{aligned}
$$

where,

- $d_{s}$ and $d_{d}$ are the antenna element spacing at the transmitter and receiver respectively,

- $\lambda$ is the wavelength of the carrier frequency,

- $\phi_{n, m}, \varphi_{n, m}$ are the departing and arriving angles of ray $m$ of cluster $n$ respectively,

- $\psi_{n, m}$ is a random initial phase of ray $m$ of cluster $n$ and

- $f_{n, m}$ is the corresponding Doppler frequency shift of ray $m$ of cluster $n$.

The LTE air interface technology supports a sampling rate of 30.72Msamples/s, which suggests a sampling time of 32.5ns. In order to generate the frequency channel gains for every sub-carrier (separated by $15 \mathrm{KHz}$ ), a FFT size of $\frac{30.72 e 6}{15 e 3}=2048$ is required. Hence, in order to find the frequency domain channel gains,

1. The cluster delays $\left(\tau_{n}\right)$ are rounded up/down to a multiple of the sampling time $T_{\text {samp }}=32.5 \mathrm{~ns}$.

2. A 2048-FFT operation is performed which yields the frequency channel information over a bandwidth of $30.72 \mathrm{MHz}$ sampled every $15 \mathrm{KHz}$.

3. The required frequency channel gains are extracted by appropriate filtering at center frequency $f_{c}$ over $\left[f_{c}-B W / 2, f_{c}+B W / 2\right]$ - The DC resource element centered at $f_{c}$ is not used for transmission. 
Monte-Carlo simulations are employed to evaluate the performance of the system. In the ITU report a drop is defined as an independent deployment of terminals and/or BSs. During the performance evaluation of a drop, the terminals and BSs are assumed not to be moving. The shadow fading effect is assumed to be constant. Moreover, the slow fading channel gains are also considered to remain unchanged. More specifically, in the simulation of a drop the parameters $P_{n}, \tau_{n}, \phi_{n, m}, \varphi_{n, m}, \psi_{n, m}$ are fixed. The time variations, however, are incorporated in a drop simulation by varying the AoA and AoD of the $N_{r}$ rays in all $N_{c}$ clusters according to the Doppler effect every sub-frame. The simulations evaluate the performance of each drop over $U$ sub-frames and are performed for many independent drops in order to evaluate the average network performance.

\subsubsection{Link spectral efficiency model}

The modulation and coding rates in the LTE release 8 standard are provided in Table 2.2. The standard supports QPSK, 16QAM and 64QAM modulation schemes and employs turbo coding. Based on simulation results, this allows this air interface technology to achieve the Shannon limit with an approximate loss of $3 \mathrm{~dB}$. The maximum capacity, however, is bounded by the highest modulation level and is equal to $5.5547 \mathrm{bits} /$ (complex dimension) for 64QAM with an approximate code rate of 0.93. Consequently, the spectral efficiency of each link in the network is modeled as (see Figure 2.6):

$$
\left.\eta=\min \left\{\log _{2}\left(1+\frac{\mathrm{SNR}}{2}\right), 5.5547\right\} \quad \text { bits/(complex dimension }\right)
$$

where, SNR denotes the signal-to-background-noise ratio.

\subsubsection{Performance metrics}

Based on the recommendations of the International Telecommunication Union (ITU) the following two performance metrics are considered in the system level evaluations [2]: 
Table 2.2: LTE supported modulations, code rates and efficiencies [1, Table 7.2.3-1]

\begin{tabular}{ccc}
\hline Modulation & Approximate code rate & $\begin{array}{c}\text { Efficiency } \\
\text { (information bits per symbol) }\end{array}$ \\
\hline QPSK & 0.076 & 0.1523 \\
QPSK & 0.12 & 0.2344 \\
QPSK & 0.19 & 0.3770 \\
QPSK & 0.3 & 0.6016 \\
QPSK & 0.44 & 0.8770 \\
QPSK & 0.59 & 1.1758 \\
16QAM & 0.37 & 1.4766 \\
16QAM & 0.48 & 1.9141 \\
16QAM & 0.6 & 2.4063 \\
64QAM & 0.45 & 2.7305 \\
64QAM & 0.55 & 3.3223 \\
64QAM & 0.65 & 3.9023 \\
64QAM & 0.75 & 4.5234 \\
64QAM & 0.85 & 5.1152 \\
64QAM & 0.93 & 5.5547 \\
\hline
\end{tabular}

Let there be a system with $N$ cells and $K$ users with a total available bandwidth of $W$. Let $b_{k}$ denote the number of correctly received bits by user $k$ (down-link) or from user $k$ (up-link) and $T$ be the time over which the data bits are received. The cell spectral efficiency, $\eta$, is then defined as:

$$
\eta=\frac{\sum_{k=1}^{K} b_{k}}{T W N} \quad(\text { bits } / \text { second } / \mathrm{Hz} / \text { cell })
$$

The maximization of the sum throughput in a cellular network would result in the maximum cell spectral efficiency. However as was discussed in Section 2.4, most of the terminals would be deprived of any service. For a proper evaluation of the system performance, it becomes important to quantify the performance of the set of users with weak channel gains as well. The cell edge spectral efficiency has been introduced as a measure of performance for this set of users. With $T_{k}$ denoting the active session time 


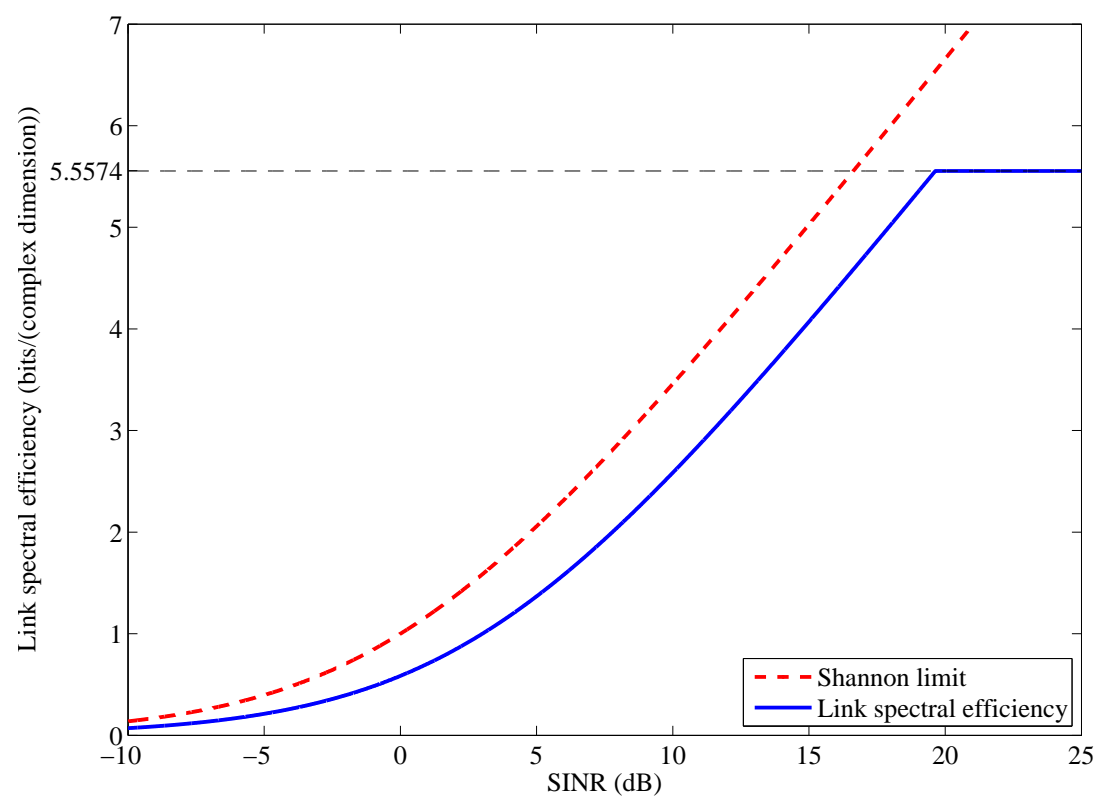

Figure 2.6: Link spectral efficiency

for user $k$, the normalized user throughput of user $k, \eta_{k}$, is defined as

$$
\eta_{k}=\frac{b_{k}}{T_{k} W} \quad(\text { bits } / \text { second } / \mathrm{Hz})
$$

The cell-edge spectral efficiency is the spectral efficiency supported by the weakest $5 \%$ of the user population and is accordingly defined as the $5 \%$ point of the cumulative distribution function of $\eta_{k}$. 


\section{Chapter 3}

\section{Localized resource allocation}

When cellular networks were first proposed, the goal was to provide a ubiquitous uniform coverage. Consequently in a traditional cellular network, the system frequency resources are uniformly distributed across the cells such that the allocated resources to the BSs are fixed and equal in size (with minimal cell breathing/channel borrowing capabilities [21]). In a modern cellular network, on the other hand, the goal is to increase the transmission rate in order to meet the ever-growing data-dominated traffic demand. In this case, the provision of a uniform network-wide coverage is not necessary, so long as the active terminals meet their service requirements. Due to the fact that the actual terminal distribution (across the cells in the network) is not uniform at all times, the resources allocated to each BS should adapt to the variations in the volume of the terminals it is serving. Hence, unlike the traditional uniform frequency allocation strategy, a localized approach should be adopted. In this chapter, a framework is presented which adapts the frequency allocations to the time-varying traffic distribution across the network.

\subsection{Scheduling cell}

Consider the cellular network with trisector cells in Figure 3.1. Each BS is positioned at the center of a cell and each cell is partitioned into 6 allocation regions. The regions 
$R_{1}, R_{2}$ and $R_{3}$ denote the cell center regions and $R_{4}, R_{5}$ and $R_{6}$ denote the cell edge regions. The cell center terminals are qualitatively defined as the terminals which receive insignificant interference from the co-channel transmissions, and the cell edge terminals are defined as the terminals who are potentially subject to excessive interference. While a lower re-use factor for the former increases the cell capacity, a higher re-use factor for the latter reduces interference on the more vulnerable terminals. In order to reduce the interference level for the cell edge terminals, no re-use is allowed across $R_{4}, R_{5}$ and $R_{6}$. Henceforth, the area covered by these regions will be referred to as the orthogonal zone.

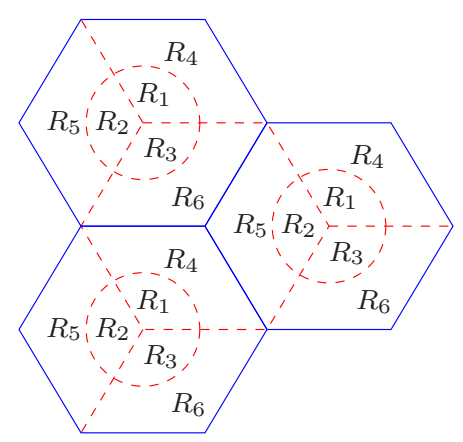

Figure 3.1: Frequency allocation regions

Soft and partial frequency reuse, SFR and PFR respectively, are two widely studied techniques in the literature. Let us first briefly study the resource allocation in these two methods:

Let $\mathcal{N}_{i}$ denote the spectrum allocated to region $i$. No re-use is allowed within any region. In $\mathrm{SFR}$, the pairs $\left(\mathcal{N}_{i}, \mathcal{N}_{i+3}\right)$ for $i=1,2,3$ form a partition of $\mathcal{N}$ and hence a frequency re-use factor of $\frac{1}{3}$ is achieved, i.e. the available spectrum is re-used three times in a cell. In PFR, the frequency resources are allocated to the regions in a slightly different manner: A portion of the spectrum, $(1-\alpha)|\mathcal{N}|$ in size, is exclusively allocated to the orthogonal zone. It is not reused in the cell center regions and is evenly distributed across the orthogonal zone regions $R_{4}, R_{5}$ and $R_{6}$. The rest of the spectrum is reused in all cell center regions. This method achieves a frequency re-use factor of $\frac{1}{1+2 \alpha}>\frac{1}{3}$, which translates into a more conservative (less) re-use compared to SFR. The two frequency 
allocation methods are illustrated in Figure 3.2.

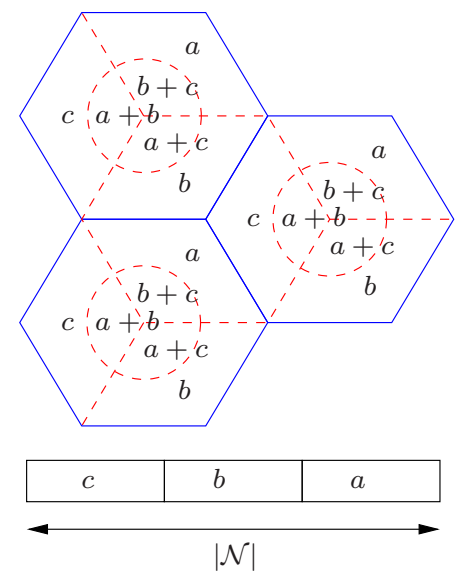

(a)
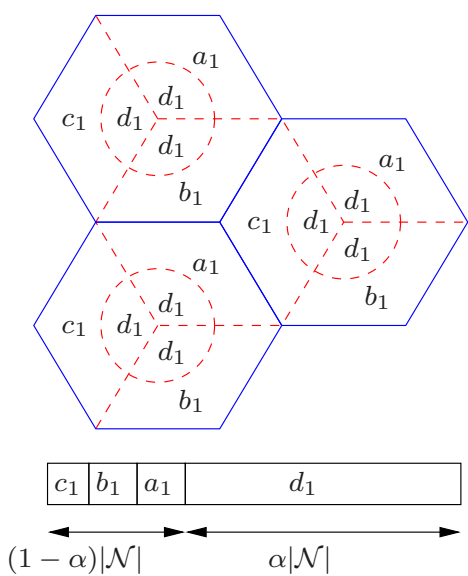

(b)

Figure 3.2: Well-known ICIC frequency allocation methods (a) Soft frequency re-use $\left(|\mathbf{a}|=|\mathbf{b}|=|\mathbf{c}|=\frac{|\mathcal{N}|}{3}\right)$, (b) Partial frequency reuse $\left(\left|\mathbf{a}_{1}\right|=\left|\mathbf{b}_{1}\right|=\left|\mathbf{c}_{1}\right|=\frac{(1-\alpha)|\mathcal{N}|}{3}\right)$

Although these techniques improve the performance, they share one major shortcoming: Consider a cellular network where SFR (or PFR) is implemented. In order to preserve the orthogonality between the orthogonal zones of adjacent cells, the frequency allocation across all cells should be identical. In other words, the same frequency blocks should be assigned to the corresponding regions of every cell in the network. And yet the traffic demand in each cell is unique, which clearly calls for a localized approach.

Definition 3.1 (Scheduling cell): Traditionally, we think of a cell as a BS which serves a set of terminals. A scheduling cell, on the other hand, is defined by a set of BSs. Qualitatively speaking, the cell comprises the set of BSs whose lack of coordination would result in high levels of interference. Resource allocation in each scheduling cell is both coordinated and autonomous. Autonomy implies independence of the resource allocation in a scheduling cell from the rest of the network, which allows a local adaptation to the traffic demand.

In this chapter, the focus will be on the coordinated resource allocation in traditional cellular networks. The scheduling cells are assumed to be static and are defined as 
follows ${ }^{1}$ :

The three BSs whose antenna beams are pointing toward one another form a scheduling cell (SC) as depicted in Figure 3.3. In this case, the BSs in a $\mathrm{SC}$ are located at three cell corners - the center of the dotted circles. In a given cell, each terminal is assigned to one BS with the strongest channel gain. The terminals located at the center of the cell (i.e. regions $R_{4}, R_{5}$ and $R_{6}$ ) experience weak channel gains from their assigned BSs. This region forms the orthogonal zone where no frequency reuse is allowed. The set of terminals in the orthogonal zone will be referred to as the orthogonal user set. The terminals located at the three corners of the $\mathrm{SC}$ (i.e. regions $R_{1}, R_{2}$ and $R_{3}$ ) form the reuse user set with more favorable channel conditions. The spectrum which has not been assigned to the orthogonal zone is utilized for simultaneous transmission from all three BSs to the reuse user set.

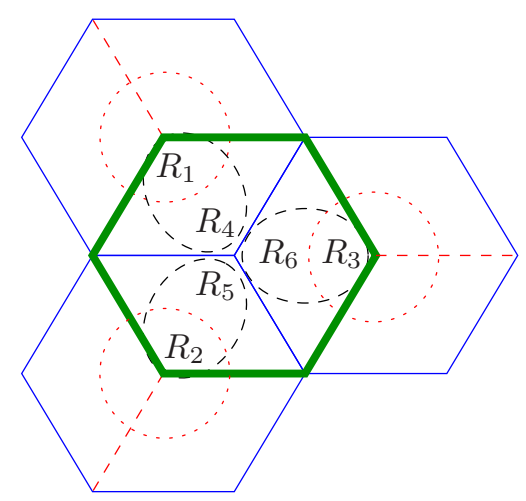

Figure 3.3: Scheduling cell

The entire spectrum, $\mathcal{N}$, is available to all SCs. Figure 3.4 illustrates how the SCs tessellate the coverage area in a cellular network. The different shades of the scheduling cells correspond to their independently chosen frequency reuse pattern. In a sense, a SC defines an autonomous resource allocation entity in the cellular network, which allows a localized approach to frequency reuse.

Before delving into the resource allocation framework, a brief study on the granularity

\footnotetext{
${ }^{1}$ Adaptive scheduling cells are discussed in chapter 4 .
} 


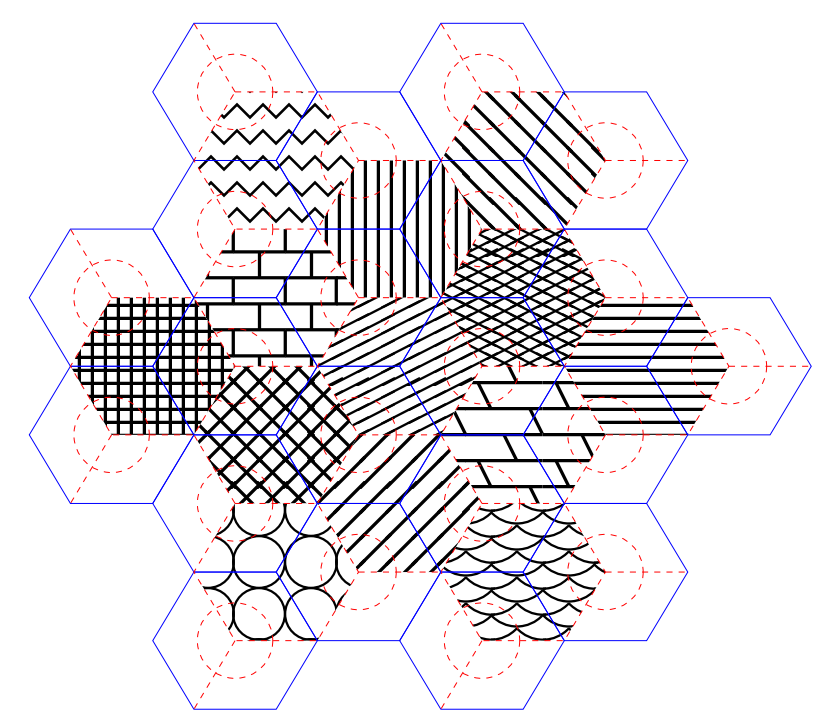

Figure 3.4: Each shaded area represents one scheduling cell.

of resource allocation is provided in Section 3.2.

\subsection{Resource allocation granularity}

In an OFDMA communication system, the smallest system resource is one OFDM symbol long and one sub-carrier wide. In LTE terminology, this resource is referred to as a resource element. A block of resource elements, 12 sub-carriers wide and 14 OFDM symbols long is referred to as a scheduling block which constitutes the smallest block of resources that can be assigned to a terminal.

The spectral efficiency of a link is not only a function of the SINR levels of the frequency channels assigned to that link, but also is affected by the level of modulation and coding scheme of each frequency channel. In this section we study different levels of granularity for the choice of the modulation and coding scheme on each sub-carrier. Three granularities are considered:

- Resource element: The modulation and coding scheme of each resource element is chosen independently according to the corresponding SINR level. 
- sub-carrier: The modulation and coding scheme of each sub-carrier (for the duration of a sub-frame) is chosen independently according to the average SINR level of the sub-carrier over the duration of one sub-frame.

- Scheduling block: The modulation and coding scheme of each resource block is chosen independently according to the average SINR level of the resource elements in one scheduling block.

- LTE: In LTE, a common modulation and coding scheme is assumed for all frequency channels assigned to each terminal. The scheme is chosen based on the average SINR level of all scheduling blocks assigned to each terminal.

Let us consider the down-link transmission of a BS with a power budget of $P_{T}$ over a spectrum of $S$ sub-carriers. Equal power level across all sub-carriers has been assumed, i.e. $p_{s}=\frac{P_{T}}{S}$, where $p_{s}$ denotes the power level on sub-carrier $s$.

\section{One resource element}

The theoretic spectral efficiency on sub-carrier $s$ and OFDM symbol $t$ denoted by resource element $(s, t)$ is

$$
\eta_{s t}=\log _{2}\left(1+\frac{h_{s t} p_{s}}{2 P_{\text {noise }}}\right) \quad \text { bits } / \text { transmission }
$$

Considering the LTE modulation and coding levels provided in Table 2.2, the spectral efficiency becomes

$$
\eta_{s t}=\digamma\left(\log _{2}\left(1+\frac{h_{s t} p_{s}}{2 P_{\text {noise }}}\right)\right) \quad \text { bits/transmission }
$$

where, $\digamma$ (.) maps the Shannon capacity with $3 \mathrm{~dB}$ penalty on the modulation and coding levels available in the LTE standard as illustrated in Fig. 3.5. 


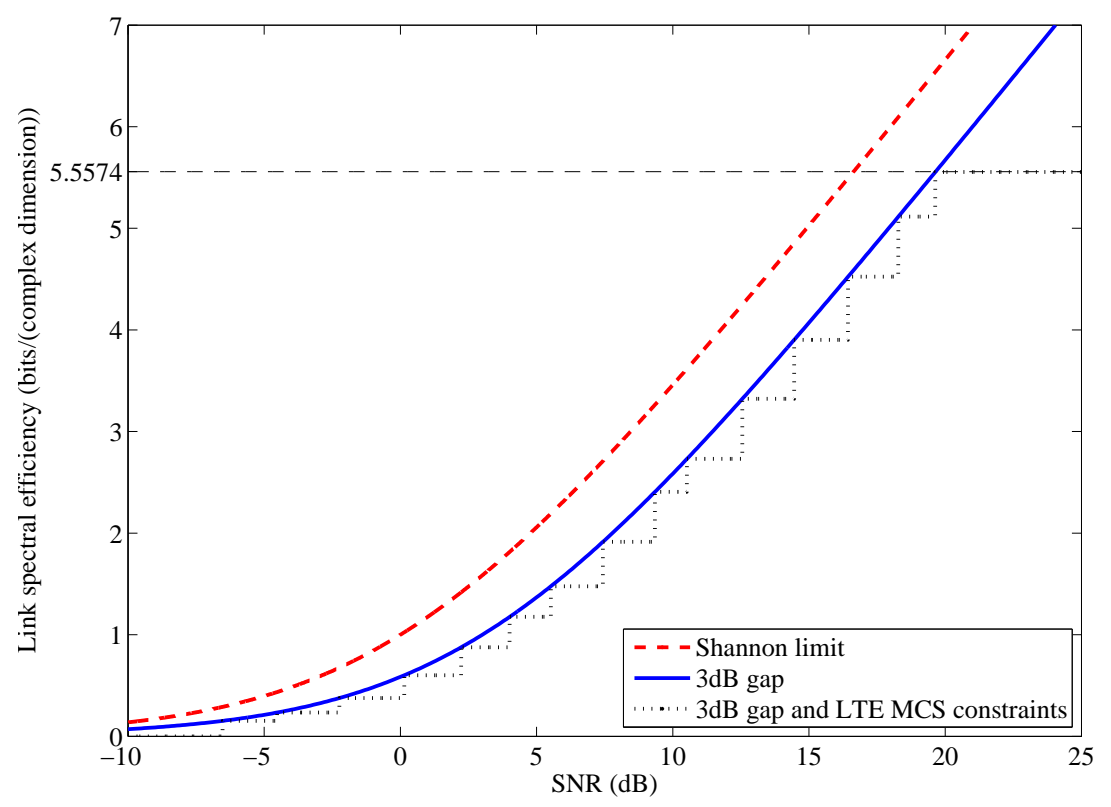

Figure 3.5: Link spectral efficiency

\section{One sub-carrier in frequency and one sub-frame in time}

The average channel gain on sub-carrier $s$ is calculated by averaging the channel gains over the 14 OFDM symbols in a sub-frame:

$$
\bar{h}_{s}=\frac{1}{14} \sum_{t=1}^{14} h_{s t}
$$

Based on the average channel gain, a common modulation and coding scheme is chosen for all the OFDM symbols in a sub-frame. Hence, the spectral efficiency on resource element $(s, t)$ is upper-bounded by:

$$
\digamma\left(\log _{2}\left(1+\frac{\bar{h}_{s} p_{s}}{2 P_{\text {noise }}}\right)\right)
$$

and the spectral efficiency of resource element $(s, t)$ can be approximated by:

$$
\eta_{s t}=\min \left\{\log _{2}\left(1+\frac{h_{s t} p_{s}}{2 P_{\text {noise }}}\right), \digamma\left(\log _{2}\left(1+\frac{\bar{h}_{s} p_{s}}{2 P_{\text {noise }}}\right)\right)\right\} \quad \text { bits/transmission }
$$




\section{One scheduling block}

A scheduling block is a block of resources 12 sub-carriers wide and 14 OFDM symbols wide which amount to 168 resource elements. The average channel gain on scheduling block $l$ is calculated by averaging the channel gains over all 168 resource elements in the block:

$$
\bar{h}_{(l)}=\frac{1}{168} \sum_{s=1}^{12} \sum_{t=1}^{14} h_{s t} \quad \text { for } \quad l=1,2, \cdots, L
$$

and the spectral efficiency for resource element $(s, t)$ can be approximated by:

$$
\eta_{s t}=\min \left\{\log _{2}\left(1+\frac{h_{s t} p_{s}}{2 P_{\text {noise }}}\right), \digamma\left(\log _{2}\left(1+\frac{\bar{h}_{(l)} p_{s}}{2 P_{\text {noise }}}\right)\right)\right\} \quad \text { bits/transmission }
$$

where $l=\left\lfloor\frac{s}{12}\right\rfloor$.

\section{All sub-carriers assigned to a terminal in frequency and one sub-frame in time (supported in LTE)}

Let $\bar{h}(k)$ be the average channel gain of all resource elements of all resource blocks assigned to user $k$. Hence the spectral efficiency of resource element $(s, t)$ can be approximated by:

$$
\eta_{s t}=\min \left\{\log _{2}\left(1+\frac{h_{s t} p_{s}}{2 P_{\text {noise }}}\right), \digamma\left(\log _{2}\left(1+\frac{\bar{h}(k) p_{s}}{2 P_{\text {noise }}}\right)\right)\right\} \quad \text { bits/transmission }
$$

\section{Thesis model}

In this thesis, it is assumed that the system resources are allocated at the granularity of one scheduling block. The link level simulation model considers the Shannon capacity with a $3 \mathrm{~dB}$ penalty for each resource element. Hence, the number of transmitted bits for 
resource element $(s, t)$ is calculated as

$$
\min \left\{\log _{2}\left(1+\frac{\mathrm{SINR}_{s t}}{2}\right), 5.5547\right\}
$$

where $\mathrm{SINR}_{s t}$ denotes the level of signal to interference plus noise ratio on the desired link over sub-carrier $s$ and OFDM symbol $t$.

\section{Numerical performance comparison}

In order to compare the performance of the studied MCS assignment granularities, the spectral efficiency of the link between a BS and one terminal over the available spectrum is studied. The system spectrum is centered at $2 \mathrm{GHz}$ with a bandwidth of $10 \mathrm{MHz}$, which amounts to 50 resource blocks. The terminal is moving at a speed of $30 \mathrm{Kmph}$. In Figure 3.6 and Figure 3.7, two snapshots of the channel across time and frequency are provided respectively .

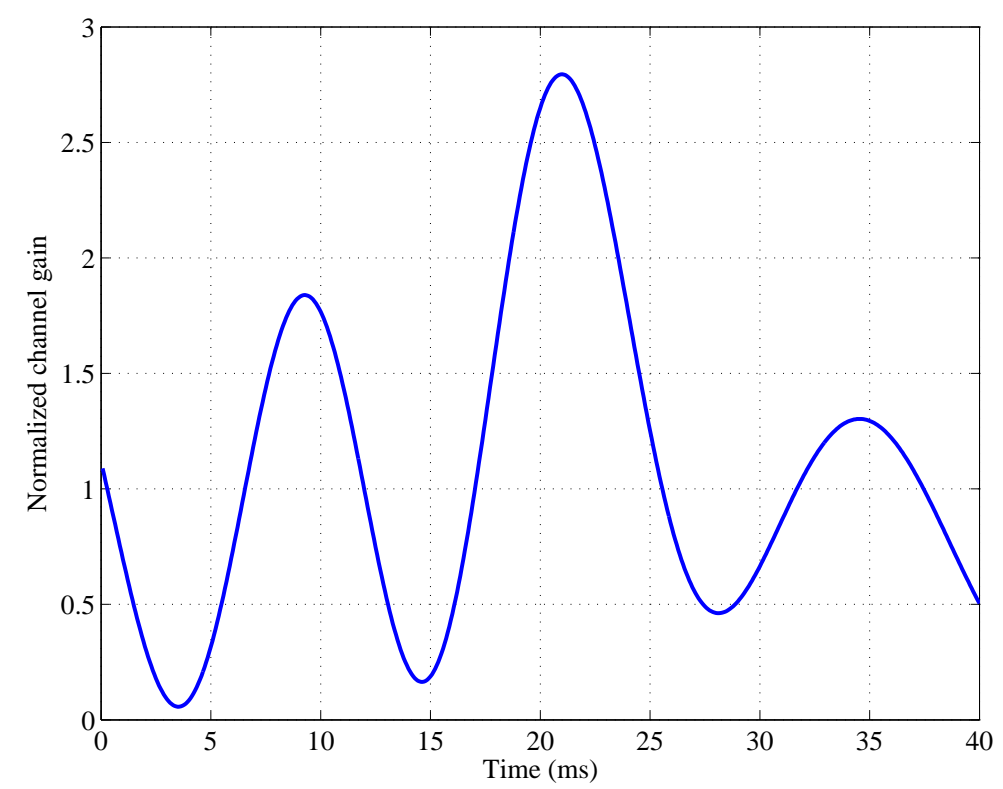

Figure 3.6: A snapshot of the wireless channel on one sub-carrier over 40 sub-frames

Figure 3.8 depicts the spectral efficiency of the system as a function of SNR. The upper-bound on the spectral efficiency corresponds to a transmission with the highest 


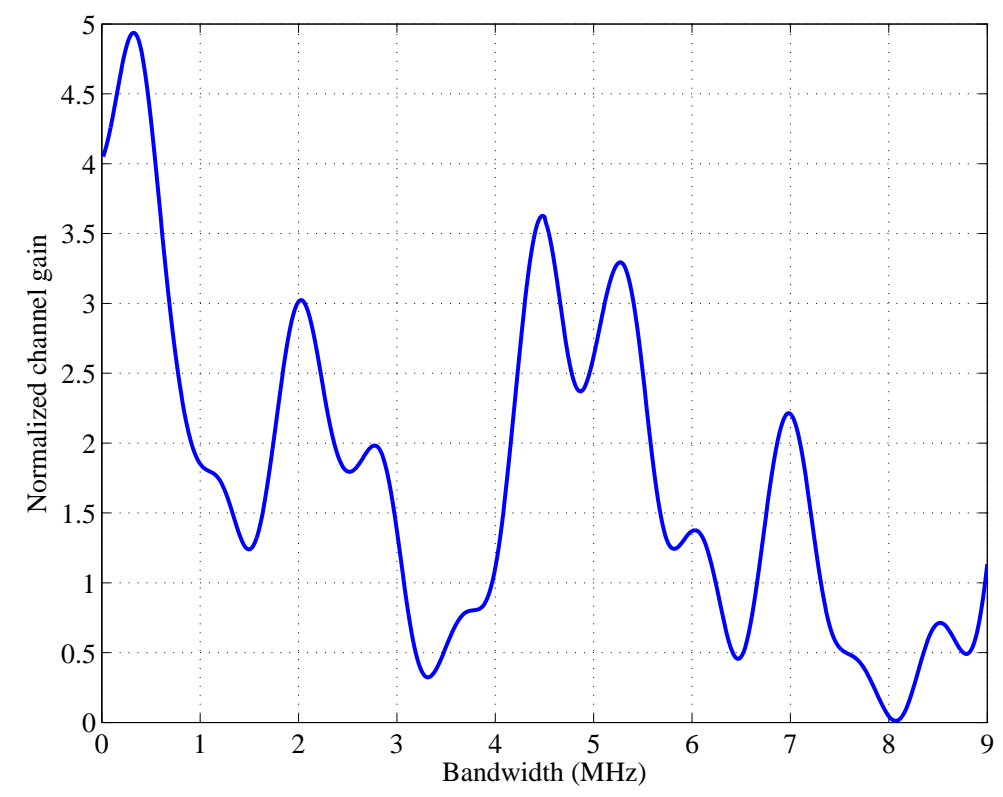

Figure 3.7: A snapshot of the wireless channel across the available spectrum

level of MCS, i.e. 5.5547 bits/transmission, on $50 \times 12 \times 14$ resource elements, which over a bandwidth of $10 \mathrm{MHz}$ results in a spectral efficiency of $4.6688 \mathrm{bps} / \mathrm{Hz}$. The results show very little difference between the studied granularities and justifies the model used in this thesis. In the rest of this chapter, system resource $n$ refers to scheduling block $n$.

\subsection{Resource allocation framework}

The localized resource allocation technique for traditional cellular networks is presented in this section. As mentioned earlier, the entire system spectrum is available for re-use in each scheduling cell. This allows an independent allocation of resources for the scheduling cells across the network.

\subsubsection{Terminal grouping}

Based on their channel conditions, the terminals in each scheduling cell are labeled as those who can tolerate in-cell interference and those who cannot. No reuse of resources 


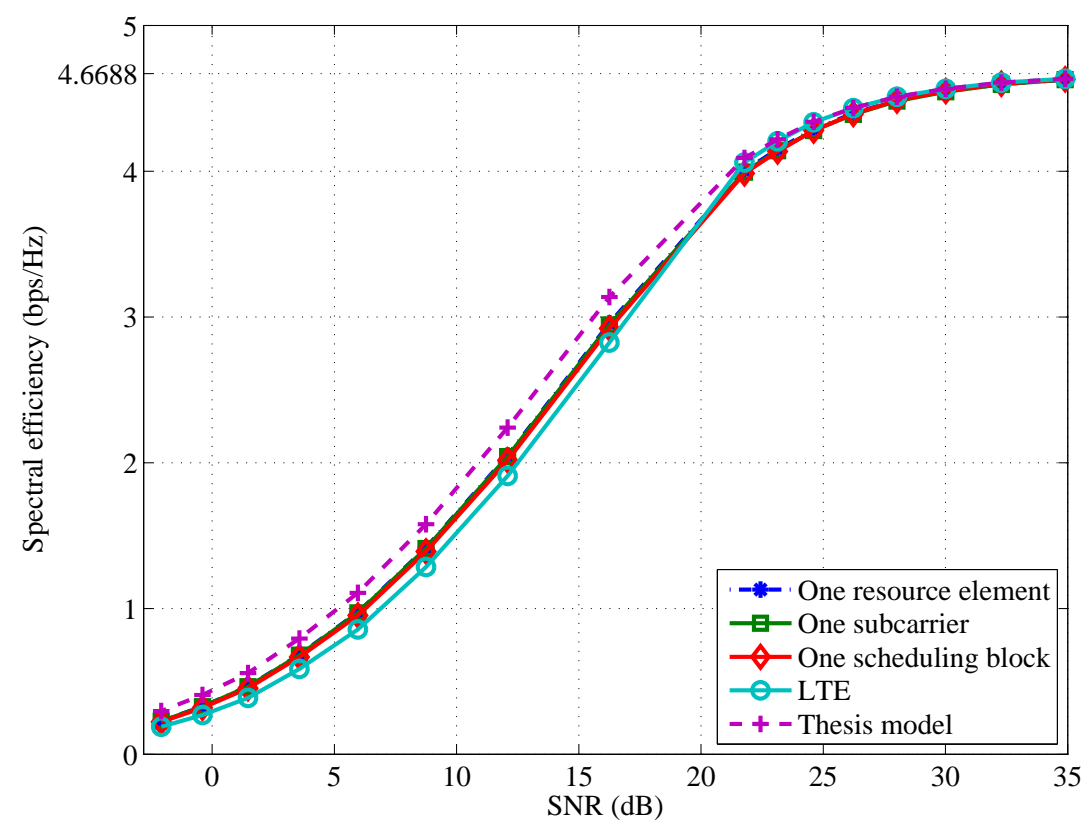

Figure 3.8: Link spectral efficiency comparison

is allowed within a scheduling cell among the terminals in the latter group to which we will refer as the orthogonal user set. The former will be referred to as the reuse user set. The terminals in this set receive interference from all BSs inside the scheduling cell. Terminal grouping is performed based on the wide-band channel gains as follows:

Definition 3.2 (Orthogonal and reuse user sets): Let $\mathcal{A}(c)$ denote the set of BSs in scheduling cell $c$ and let $h_{l m}$ be the channel gain between terminal $l$ and BS $m$. Terminal $l$ assigned to BS $i$ belongs to the orthogonal user set if

$$
\frac{h_{l i}}{\sum_{m \neq i, a_{m} \in \mathcal{A}(c)} h_{l m}}<\alpha
$$

where $a_{m}$ denotes BS $m$. And terminal $l$ belongs to the reuse user set if

$$
\frac{h_{l i}}{\sum_{m \neq i, a_{m} \in \mathcal{A}(c)} h_{l m}} \geq \alpha
$$

All BSs in the scheduling cell transmit independent data streams on the resources allocated to the reuse user set simultaneously. In order to avoid excessive interference 
especially to the out-of-cell terminals, we introduce a power reduction factor $0<\beta<1$. As a result, while the transmit power of $\mathrm{BS} i$ to the orthogonal user set is $p_{i}$, the transmit power to the reuse user set is reduced to $\beta p_{i}$. This power control strategy is studied in detail in section 3.3.3.

The resource allocation in a scheduling cell is performed in the following two steps:

\subsubsection{Step 1 - User set resource allocation}

Due to the nature of the user sets, the resources allocated to one set cannot be reused for the other set. Let $\mathcal{K}(c)$ denote the set of terminals assigned to the BSs in scheduling cell c. Given the BS set $\mathcal{A}(c)$ and the user set $\mathcal{K}(c)$, in this step the spectrum is partitioned into the orthogonal resource set, $\mathcal{N}_{c}^{(O)}$, and reuse resource set, $\mathcal{N}_{c}^{(R)}$, such that:

- Resource $n \in \mathcal{N}_{c}^{(O)}$ is assigned to exactly one terminal in the orthogonal user set $K_{c}^{(O)}$ per TTI.

- Resource $n \in \mathcal{N}_{c}^{(R)}$ is assigned to exactly one terminal in the reuse user set $K_{c}^{(R)}$ per BS per TTI.

It is important to note that the resource set allocation is performed at a time scale larger than a $\mathrm{TTI}^{2}$ and should adapt to the volume of the traffic demand in each user set. The size of the resource set allocated to each user set is chosen to be proportional to the size of the corresponding user set, i.e.

$$
\frac{\left|\mathcal{N}_{c}^{(O)}\right|}{\left|\mathcal{N}_{c}^{(R)}\right|}=\frac{\left|\mathcal{K}_{c}^{(O)}\right|}{\left|\mathcal{K}_{c}^{(R)}\right|}
$$

In a scheduling cell with the user set $\mathcal{K}(c)$ and the available spectrum of $\mathcal{N}$, we have,

$$
\left|\mathcal{N}_{c}^{(O)}\right|+\left|\mathcal{N}_{c}^{(R)}\right|=|\mathcal{N}| \quad, \quad\left|\mathcal{K}_{c}^{(O)}\right|+\left|\mathcal{K}_{c}^{(R)}\right|=|\mathcal{K}(c)|
$$

\footnotetext{
${ }^{2}$ The different time scales of adaptation in the proposed resource allocation framework are discussed in Section 3.3.5.
} 
Using (3.12) and (3.13), the size of the resource sets are readily available.

$$
\left|\mathcal{N}_{c}^{(O)}\right|=\frac{\left|\mathcal{K}_{c}^{(O)}\right|}{|\mathcal{K}(c)|} \cdot|\mathcal{N}| \quad, \quad\left|\mathcal{N}_{c}^{(R)}\right|=\frac{\left|\mathcal{K}_{c}^{(R)}\right|}{|\mathcal{K}(c)|} \cdot|\mathcal{N}|
$$

Thus the user sets are allocated a percentage of the system resources proportional to the size of the corresponding user set. As an example, if $80 \%$ of the terminals in the scheduling cell reside in the orthogonal user set (and consequently $20 \%$ reside in the reuse user set), $80 \%$ of the system resources are assigned to the orthogonal user set and $20 \%$ of the system resources are assigned to the reuse user set.

Finally, in order to exploit frequency diversity, each resource set is formed by randomly choosing the corresponding number of resources from the available pool of resources. It is important to note that resource set allocation is performed independently in each scheduling cell according to the traffic pattern in the corresponding cell.

It should be noted that at this stage, the fairness of the resource allocation method cannot be evaluated solely based on the number of resources assigned to the terminal sets. Firstly, the terminal scheduling occurs at the second stage of the framework and will be discussed in section 3.3.4. More importantly, due to an intrinsic property of the wireless channel, fairness cannot be defined merely based on the number of resources assigned to each terminal. The assignment of the same frequency resource to two terminals can bring very different utilities to those terminals. This is due to the very different channel gains and interference levels the two terminals can experience at the same time. Although this has been a sensible definition in wired networks - since all terminals experience more or less the same channel conditions - it cannot be extended to a wireless communication system. Intricate discussions on how fairness should be viewed in a wireless network has been provided in section 2.4. The fairness of the proposed resource allocation framework will be evaluated based on the average data rates achieved by the terminals in the network over a sufficiently long period of time. 


\subsubsection{Power control strategy}

Based on the terminal grouping discussed in section 3.3.1, the terminals in scheduling cell $c$ are partitioned into the orthogonal and reuse user sets $\mathcal{K}_{c}^{(O)}$ and $\mathcal{K}_{c}^{(R)}$ respectively. Similarly, the system spectrum $\mathcal{N}$ is partitioned into the orthogonal and reuse resource sets $\mathcal{N}_{c}^{(O)}$ and $\mathcal{N}_{c}^{(R)}$. In the proposed framework, two transmission power levels are considered. The transmission power level of $\mathrm{BS} i$, denoted by $p_{i}$, is equal to $p_{i}^{(O)}$ for a transmission to a terminal in the orthogonal user set and is equal to $p_{i}^{(R)}$ for a transmission to a terminal in the reuse user set. The choice of the two power levels are discussed below.

In scheduling cell $c$, let us assume terminal $l$ is assigned to BS $i$ and is a member of the orthogonal user set. The transmission from BS $i$ to terminal $l$ occurs on resource(s) $n \in \mathcal{N}_{c}^{(O)}$. The other BSs in the scheduling cell are not allowed to simultaneously transmit on the same resource. In other words, the terminals in the orthogonal user set do not experience interference from the BSs in the scheduling cell. The quality of these links, however, are affected by the interference from the other scheduling cells in the network whose resource allocation (transmissions) are not coordinated with scheduling cell $c$. Hence, the SINR of terminal $l$ is

$$
\frac{h_{l i} p_{i}^{(O)}}{I_{c}+P_{N}}
$$

where $h_{l i}$ is the channel gain between BS $i$ and terminal $l, p_{i}^{(O)}$ denotes the power level for the transmission to a terminal in the orthogonal set, $I_{c}$ is the out-of- scheduling cell interference and $P_{N}$ is the background noise power level.

Let us consider the other case, where in scheduling cell $c$ terminal $i$ assigned to BS $i$ is a member of the reuse set. In this case, the transmission from BS $i$ to terminal $l$ occurs on resource(s) $n \in \mathcal{N}_{c}^{(R)}$. The BS with active terminals in the scheduling cell, all simultaneously transmit on the same resources. This in turn translates into in-cell 
interference for all terminals in the reuse set. The SINR of terminal $l$ is

$$
\frac{h_{l i} p_{i}^{(R)}}{i_{c}+I_{c}+P_{N}}
$$

where $p_{i}^{(R)}$ is the power level for the transmission to the reuse terminals and $i_{c}$ denotes the in-cell interference level.

Assuming that $p_{0}$ is the transmission power budget for a $\mathrm{BS}$ on a given frequency resource, we choose the orthogonal transmission power level $p_{i}^{(O)}$ to be equal to $p_{0}$ and the reuse transmission power level $p_{i}^{(R)}$ to be equal to $\beta p_{0}$, where $\beta$ is a system parameter between zero and one. With $\beta=1$, all transmissions occur at the same power level and essentially no power control is enforced. In order to study the effect of decreasing $\beta$, let us consider the SINR level of a terminal in the orthogonal set in more detail.

$$
\frac{h_{l i} p_{0}}{I_{c}+P_{N}}
$$

The interference emanating from out of the scheduling cell, $I_{c}$, can be decomposed into the reuse and orthogonal transmissions, i.e. $I_{c}=I_{c}^{(O)}+I_{c}^{(R)}$. Hence the SINR level can be re-written as:

$$
\frac{h_{l i} p_{0}}{I_{c}^{(R)}+I_{c}^{(O)}+P_{N}}
$$

Decreasing $\beta$, scales down $I_{c}^{(R)}$ and improves the quality of all terminals in the orthogonal user sets in the network.

The effect of the system parameter $\beta$ on the quality of the links of the reuse user set terminals is a bit more complicated. For a terminal in the reuse set, the SINR is

$$
\frac{h_{l i} \beta p_{0}}{i_{c}+I_{c}^{(R)}+I_{c}^{(O)}+P_{N}}
$$

Changing the value of $\beta$ scales the desired received power, and the interference from the 
reuse transmissions by the same factor. Since all terms in the SINR except for $I_{c}^{(O)}+P_{N}$ will be scaled by the same factor, decreasing $\beta$ essentially increases the effect of the orthogonal transmission interference $\left(I_{c}^{(O)}\right)$ and the background noise $\left(P_{N}\right)$ on the link quality. Hence depending on the traffic patterns in the network, varying $\beta$ can have different effects on the quality of the link of the reuse user set terminals. In a network with small orthogonal user sets, varying $\beta$ will have little to no effect on the quality of these links. On the other hands, in a network where the majority of the terminal population resides in the orthogonal user sets decreasing $\beta$ can significantly degrade the link quality.

In this thesis, a constant value of 0.5 has been considered for the system parameter $\beta$. In the design of an actual network, however, the value for this parameter can be adjusted adaptively based on the varying network traffic.

\subsubsection{Step 2 - Terminal scheduling}

At each TTI, the orthogonal and reuse terminals are scheduled on their corresponding

resource sets based on the resource mappings $\mathcal{N}_{c}^{(O)} \longrightarrow \mathcal{K}_{c}^{(O)}$ and $\mathcal{N}_{c}^{(R)} \longrightarrow \mathcal{K}_{c}^{(R)}$ respectively.

\section{Reuse user set scheduling}

Let $\mathcal{K}^{(R)}(i)$ be the set of reuse terminals assigned to BS $i$. The available spectrum, $\mathcal{N}_{c}^{(R)}$, is reused at all BSs $a \in \mathcal{A}(c)$. The instantaneous data rate of terminal $l$ when assigned $\mathrm{SB} j$ in sub-frame $t$ is

$$
r_{l j}(t)=\frac{168}{1 \mathrm{~ms}} \log _{2}\left(1+\frac{\overline{\mathrm{SINR}}_{l j}(t)}{2}\right) \quad \mathrm{bits} / \mathrm{s} \quad, \quad k_{l} \in \mathcal{K}_{c}^{(R)}
$$


The $\overline{\operatorname{SINR}}_{l j}(t)$ is defined as

$$
\overline{\operatorname{SINR}}_{l j}(t)=\frac{1}{168} \sum_{r=1}^{168} \frac{p_{i} g_{l i j r}(t)}{\sum_{a_{m} \in \mathcal{A}(c)-\left\{a_{i}\right\}} p_{m} g_{l m j r}(t)+\eta_{0} W} \quad, \quad k_{l} \in \mathcal{K}_{c}^{(R)}
$$

where $g_{l m j r}(t)$ is the time-varying channel gain between terminal $l$ and BS $m$ for resource element $r$ of SB $j . \overline{\mathrm{SNR}}_{l j}$ is similarly defined as the average signal-to-noise ratio at terminal $l$ in the orthogonal user set on $\mathrm{SB} j$.

The average rate of each terminal is initialized to an arbitrarily small value and is updated after resource allocation in sub-frame $t$ as

$$
\bar{r}_{l}(t)=\left(1-\frac{1}{t}\right) \bar{r}_{l}(t-1)+\frac{1}{t} \sum_{n_{j} \in \mathcal{N}} r_{l j}(t) I_{l j}(t)
$$

where $I_{l j}(t)$ is equal to one if $\mathrm{SB} j$ is assigned to terminal $l$ in sub-frame $t$ and zero otherwise.

A proportional fair scheduler with partial updating (PFS-PU) allocates the system resources in sub-frame $t$ as outlined in Algorithm 1. The algorithm runs in all BSs independently.

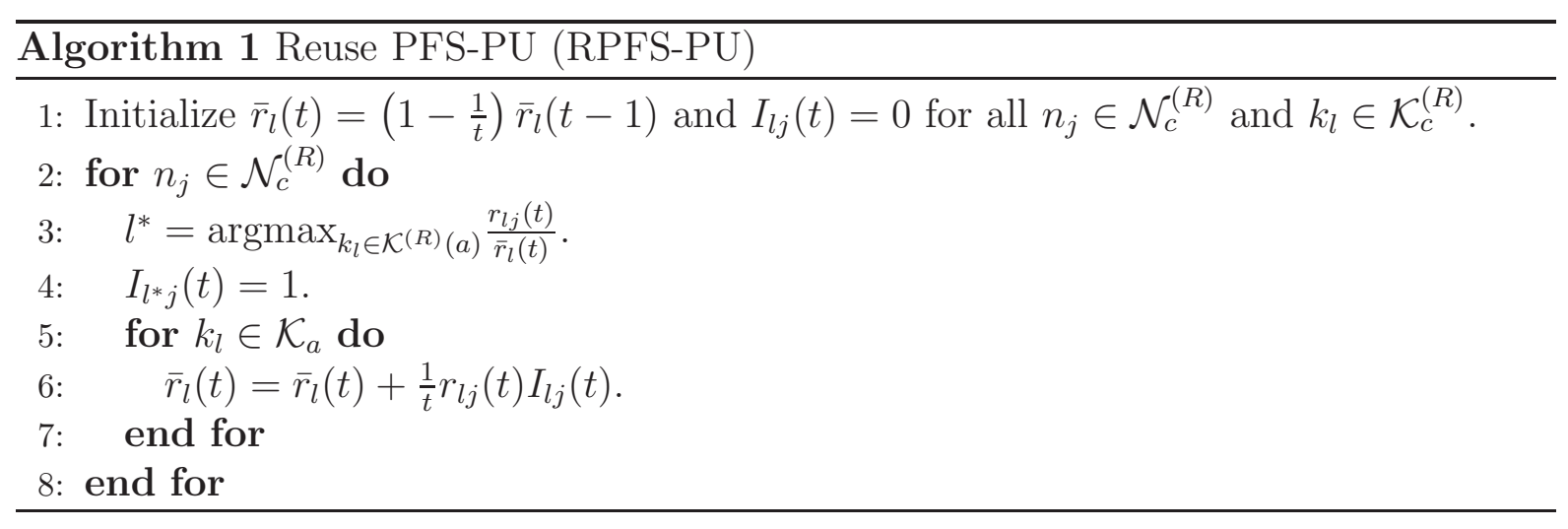

The following remarks should be noted:

- In this thesis a backlogged traffic model has been assumed. With a different traffic model, some modifications to this algorithm are required which is out of the scope 
of this work.

- If a BS in the scheduling cell has an empty reuse user set, no transmissions occur on the reuse resource set frequency bands from that BS. This decreases the level of the in-cell interference for the reuse user set terminals and the out-of-cell interference levels for the other scheduling cells in the network.

\section{Orthogonal user set scheduling}

A coordinated proportional fair scheduler among the BSs $a \in \mathcal{A}(c)$ allocates the system resources, $\mathcal{N}_{c}^{(O)}$, to the orthogonal user set. Similar to (3.20), the instantaneous data rate of terminal $l$ on $\mathrm{SB} j$ in sub-frame $t$ is

$$
r_{l j}(t)=\frac{168}{1 \mathrm{~ms}} \log _{2}\left(1+\frac{\overline{\mathrm{SNR}}_{l j}(t)}{2}\right) \quad \mathrm{bits} / \mathrm{s} \quad, \quad k_{l} \in \mathcal{K}_{c}^{(O)}
$$

The scheduler is similar to RPFS-PU, with the difference that resource allocation is coordinated between the BSs and no frequency re-use is allowed. Algorithm 2 runs at one of the BSs (or a central controller) in each scheduling cell.

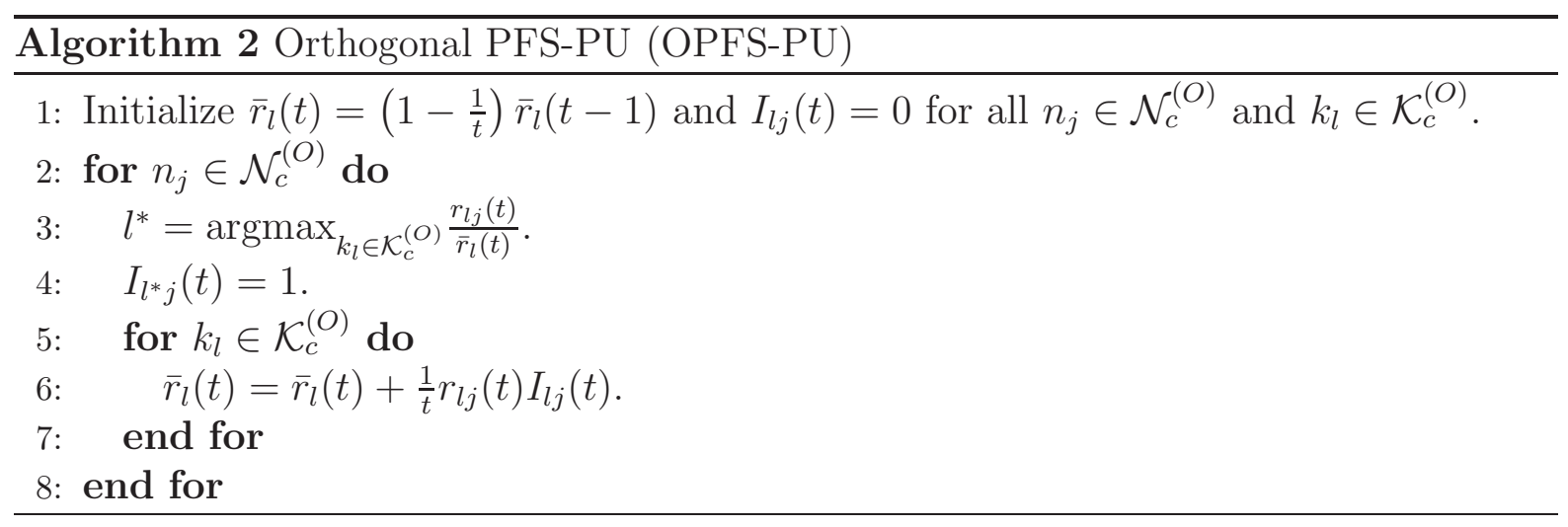

\subsubsection{Time scales of adaptation}

The proposed resource allocation framework is comprised of three levels:

- Level A: Terminal grouping 
- Level B: Resource set allocation

- Level C: Terminal scheduling

While a static set of resources would result in an inefficient resource allocation, a very fast rate of adaptation would require a tremendous amount of signaling overhead and would be impractical. The resource sets are proposed to be updated at the rate of variations in the large-scale fading channel gains. If we assume shadow fading changes with a displacement in the order of tens of wavelengths, for a system with a center frequency of $2 \mathrm{GHz}$ and terminal velocity of $30 \mathrm{Kmph}$, a terminal travels ten wavelengths in 0.18 seconds which is equivalent to 18 frames in the LTE system. Thus, we assume a typical value of $r_{A}=10$ updates per second (i.e. once every 10 frames). Terminal scheduling occurs at the smallest resource allocation granularity i.e. once every TTI. In the LTE system, a TTI is defined as a sub-frame of $T_{s}=1 \mathrm{~ms}$. Consequently, the resources are allocated at the rate of $r_{C}=\frac{1}{T_{s}}$.

While wide-band channel state information (CSI) is sufficient for terminal grouping, a more detailed frequency selective CSI is required for resource allocation. In Table 3.1, the required CSI for each level is provided. The CSI at each level is updated at the rate of resource allocation of the corresponding level. Compared to level $\mathrm{C}$, the slower rate of resource allocation at level B results in a slower update of the CSI at that level. This is due to the fact that while slow fading frequency selective information is sufficient for resource allocation at level $\mathrm{B}$, the fast fading information is required for terminal scheduling at level C.

Table 3.1: Channel model

\begin{tabular}{|c|c|c|}
\hline Resource allocation level & Required CSI & CSI update rate \\
\hline \hline Level A & Wide-band (Path-loss+Shadow fading) & $r_{A}$ \\
\hline Level B & Frequency selective (Path-loss+Shadow fading+Multi-path fading) & $r_{B}$ \\
\hline Level C & Frequency selective (Path-loss+Shadow fading+Multi-path fading) & $r_{C}$ \\
\hline
\end{tabular}


In each scheduling cell, the coordinated resource allocation takes place at a central control unit - which can be one of the BSs forming the scheduling cell. The required channel state information is collected at each BS and communicated to the central unit through the backbone connection. The decisions taken at the central unit are reported back to the BSs through the backbone connection. This requires a fast on-line exchange of information through the wired backbone network between the central unit and the BSs. Although the implementation of the backbone network is not trivial and may be costly at times, it is also not the bottleneck in the design of the resource allocation frameworks for cellular networks and hence is out of the scope of this work.

\subsection{Numerical results}

Simulation parameters are provided in Table 3.2. The BS power budget is evenly distributed across all sub-carriers, which amounts to a power budget of $18.22 \mathrm{dBm}$ per subcarrier. The directional antennas at the BSs have the following beam-pattern:

$$
A(\theta)=-\min \left[12\left(\frac{\theta}{\theta_{3 \mathrm{~dB}}}\right)^{2}, A_{\mathrm{max}}\right]
$$

where $\theta$ is calculated from the bore-sight direction of the corresponding sector, $A_{\max }=$ $20 \mathrm{~dB}$ is the maximum attenuation and $\theta_{3 \mathrm{~dB}}=70^{\circ}$ is the $3 \mathrm{~dB}$ beam-width. The beampattern is provided in Figure 3.9.

A traditional cellular network with the regular hexagonal pattern of BS deployment is considered. The system performance of a scheduling cell is evaluated in the presence of two rings of interfering cells. The following two schemes have been considered for comparison:

- UNiVERSAL FREQUENCY REUSE (UFR): In this scheme the cells are not sectorized. The system spectrum is available for simultaneous transmission to all BSs. 


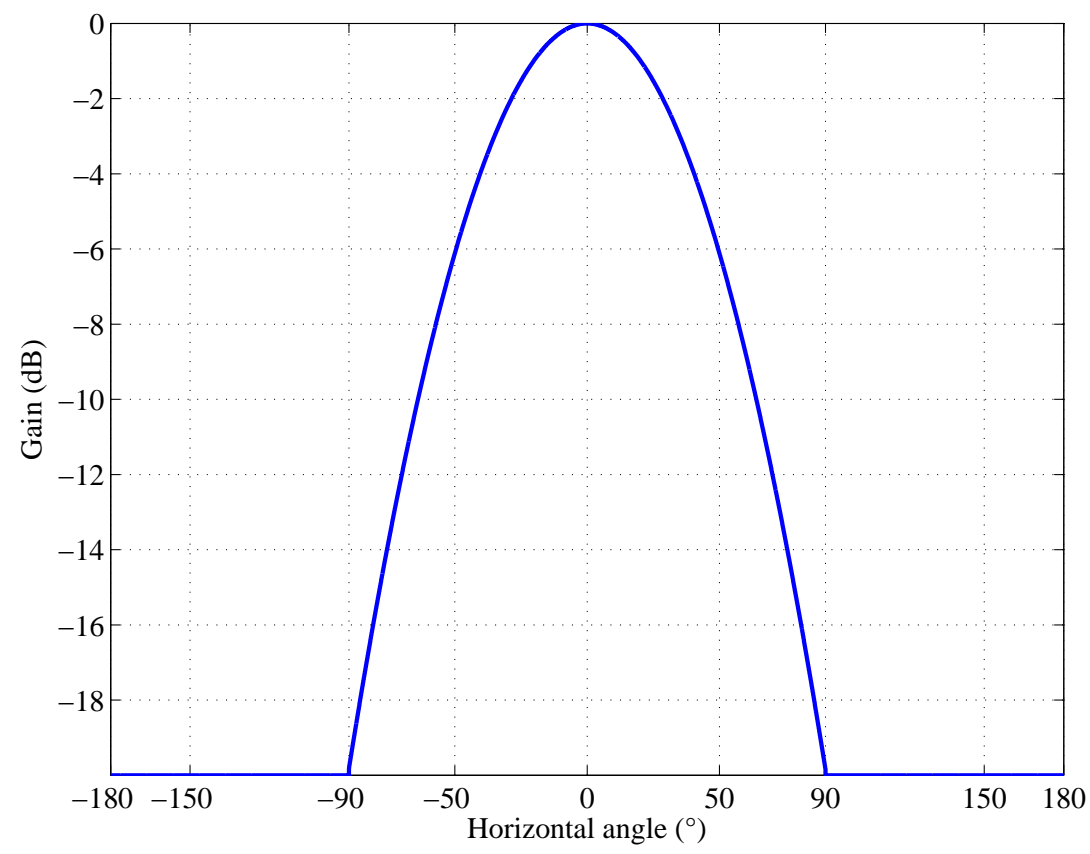

Figure 3.9: Antenna beam patter for 3-sector cells

- Partial fRequency Reuse (PFR): The partial frequency reuse strategy in Figure 3.2(b) is employed. The resources assigned to each region are fixed regardless of the actual traffic pattern. It is assumed that $25 \mathrm{SBs}$ are assigned to the reuse resource set (region $d_{1}$ in the figure) and the remaining 25 SBs are equally distributed across the three orthogonal regions $a_{1}, b_{1}$ and $c_{1}$ such that they are allocated 8,8 and 9 SBs respectively ${ }^{3}$.

For a fair comparison, the same PF scheduler is considered for all three schemes. In addition, while the cell edge (orthogonal) transmissions occur at $18.22 \mathrm{dBm}$ per subcarrier, the center terminals (reuse set users) are served with half of the allocated power budget i.e. $15.21 \mathrm{dBm}$.

In a cellular network, the traffic pattern is determined by the distribution of the active terminals across the network. Given a value for $\alpha$, different traffic patterns result in different sizes of the user sets. Similarly with the same traffic pattern, changing $\alpha$

\footnotetext{
${ }^{3}$ This choice of spectrum partitioning for FFR is arbitrary.
} 
Table 3.2: System parameters

\begin{tabular}{|c|c|}
\hline System parameter & Value \\
\hline \hline Carrier frequency & $2 \mathrm{GHz}$ \\
\hline Bandwidth & $10 \mathrm{MHz}$ \\
\hline Terminal velocity & $30 \mathrm{Kmph}$ \\
\hline BS power budget & $46 \mathrm{dBm}$ \\
\hline BS antenna gain & $17 \mathrm{~dB}$ \\
\hline Noise figure at terminal & $7 \mathrm{~dB}$ \\
\hline Background noise & $-174 \mathrm{dBm} / \mathrm{Hz}$ \\
\hline Traffic model & Full buffer \\
\hline Power reduction factor $(\beta)$ & 0.5 \\
\hline
\end{tabular}

will change the sizes of the user sets. Figure 3.10 illustrates the average percentage of terminals in each user set as a function of $\alpha$. As expected, with a higher $\alpha$, more terminals are assigned to the orthogonal user set which in turn reduces the frequency reuse in the network.

In this chapter a uniform distribution for the terminals is considered. However, in order to evaluate the performance of the proposed scheme under different traffic patterns, the size of the user sets are manipulated by changing the value for $\alpha$. Hence, three traffic distributions provided in Table 3.3 are considered. The three cases can be viewed as the traffic pattern of three scheduling cells in the network.

Table 3.3: Three scenarios

\begin{tabular}{|c|c|c|c|}
\hline $\begin{array}{c}\text { Traffic } \\
\text { distribution pattern }\end{array}$ & $\alpha(\mathrm{dB})$ & $\begin{array}{c}\text { Reuse } \\
\text { user set population (\%) }\end{array}$ & $\begin{array}{c}\text { Orthogonal } \\
\text { user set population (\%) }\end{array}$ \\
\hline (a) & 1.9 & 90 & 10 \\
\hline (b) & 14 & 50 & 50 \\
\hline (c) & 38 & 10 & 90 \\
\hline
\end{tabular}

The cell average and cell edge spectral efficiencies for distribution pattern (a) are provided in Figure 3.11 and Figure 3.12 respectively. The spectral efficiencies for distribution patterns (b) and (c) are provided in Figures 3.13 - 3.16. 


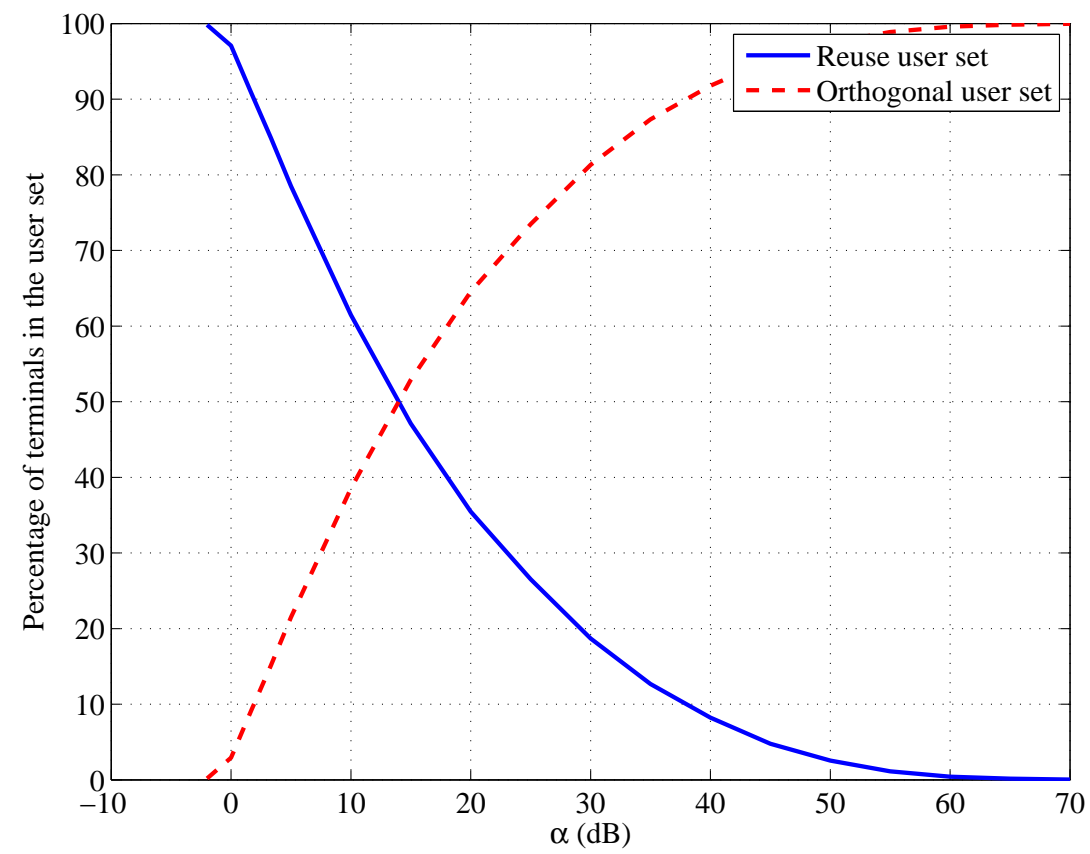

Figure 3.10: Effect of $\alpha$ on terminal grouping

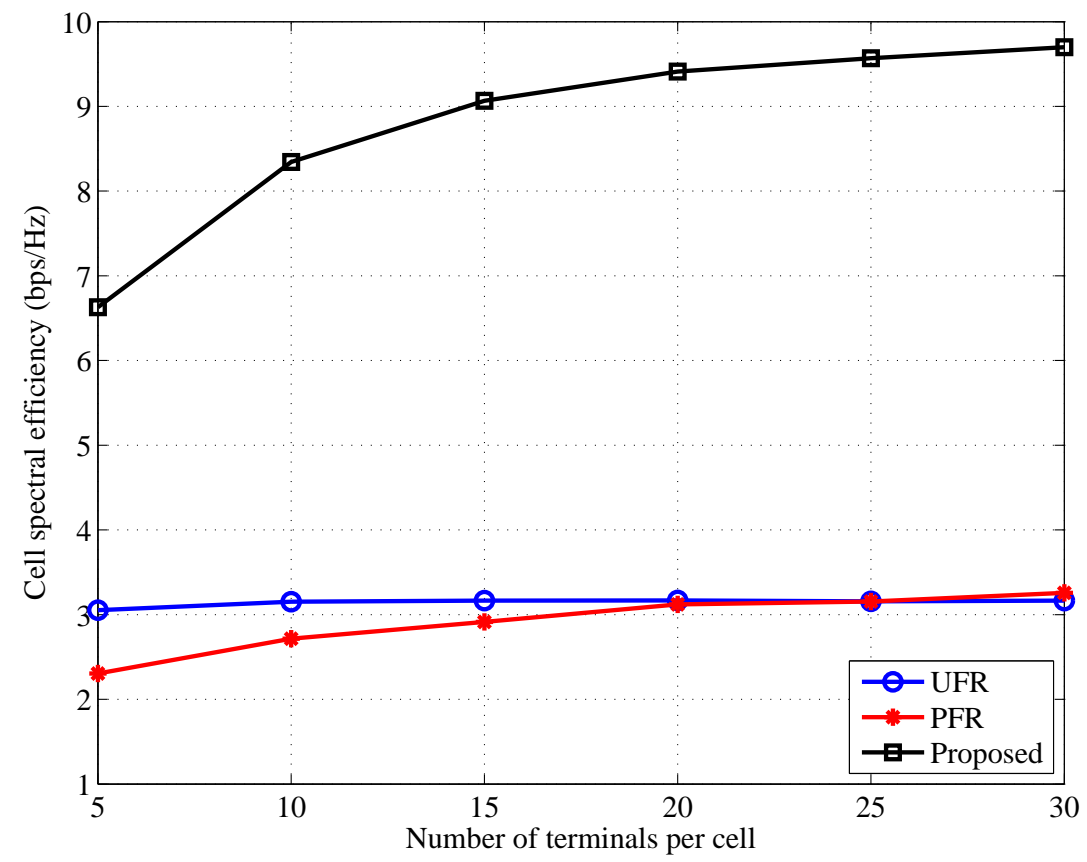

Figure 3.11: Cell spectral efficiency comparison for distribution pattern (a) 


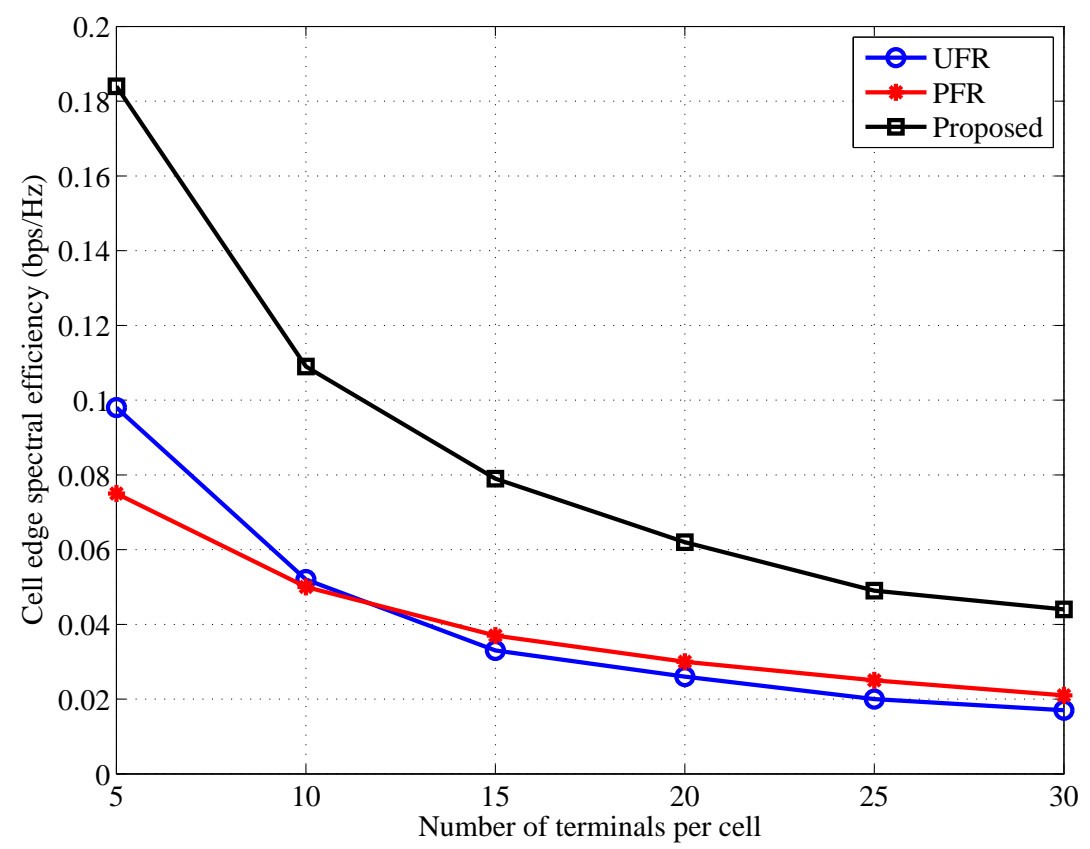

Figure 3.12: Cell edge spectral efficiency comparison for distribution pattern (a)

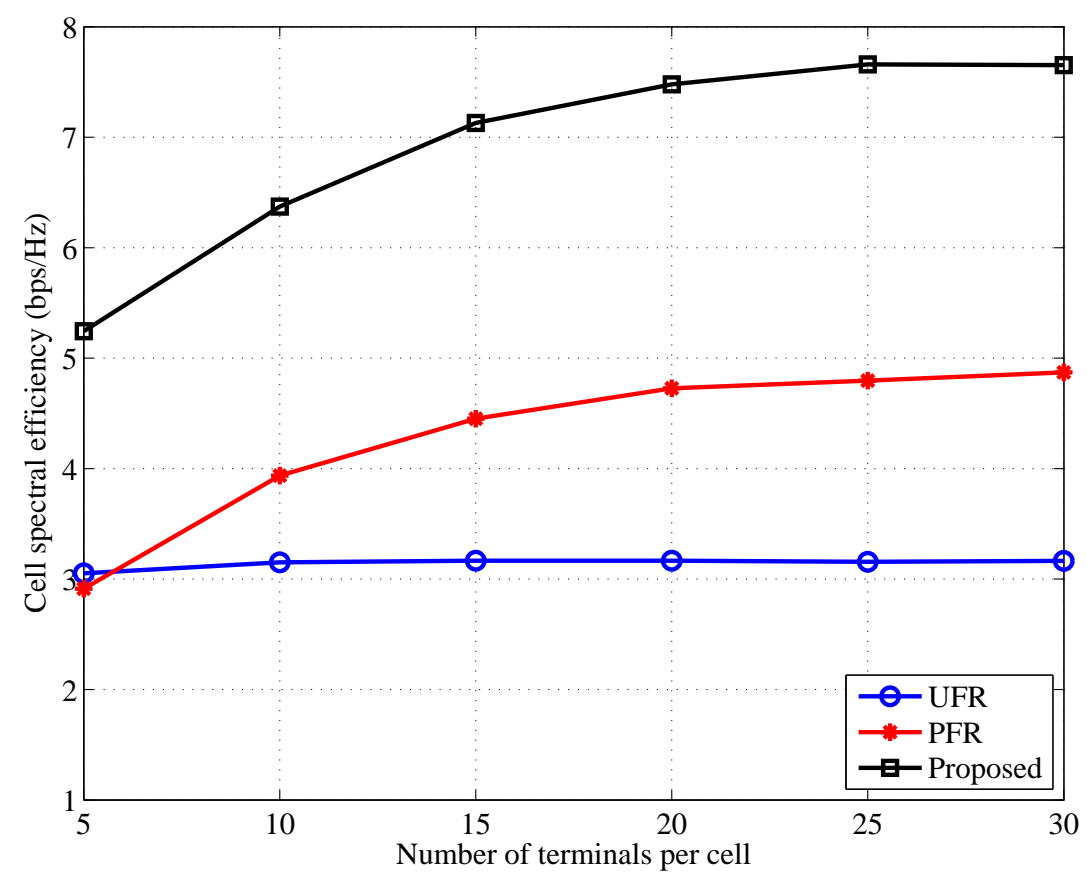

Figure 3.13: Cell spectral efficiency comparison for distribution pattern (b) 


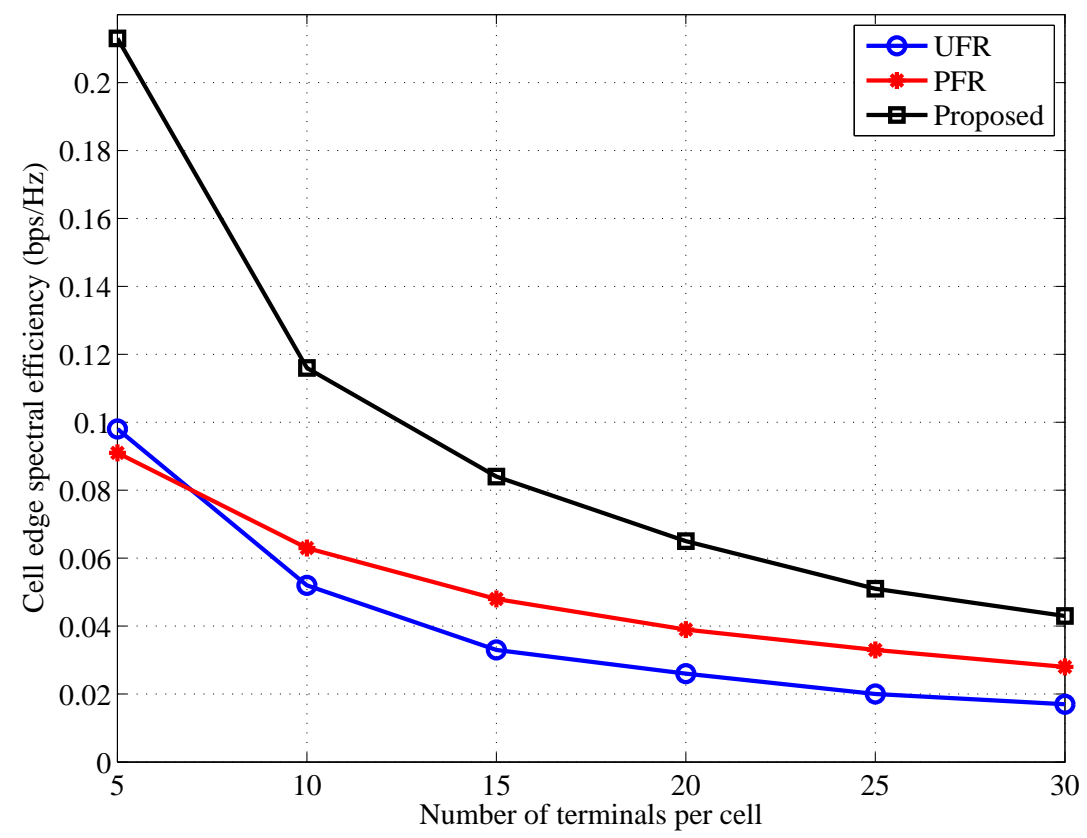

Figure 3.14: Cell edge spectral efficiency comparison for distribution pattern (b)

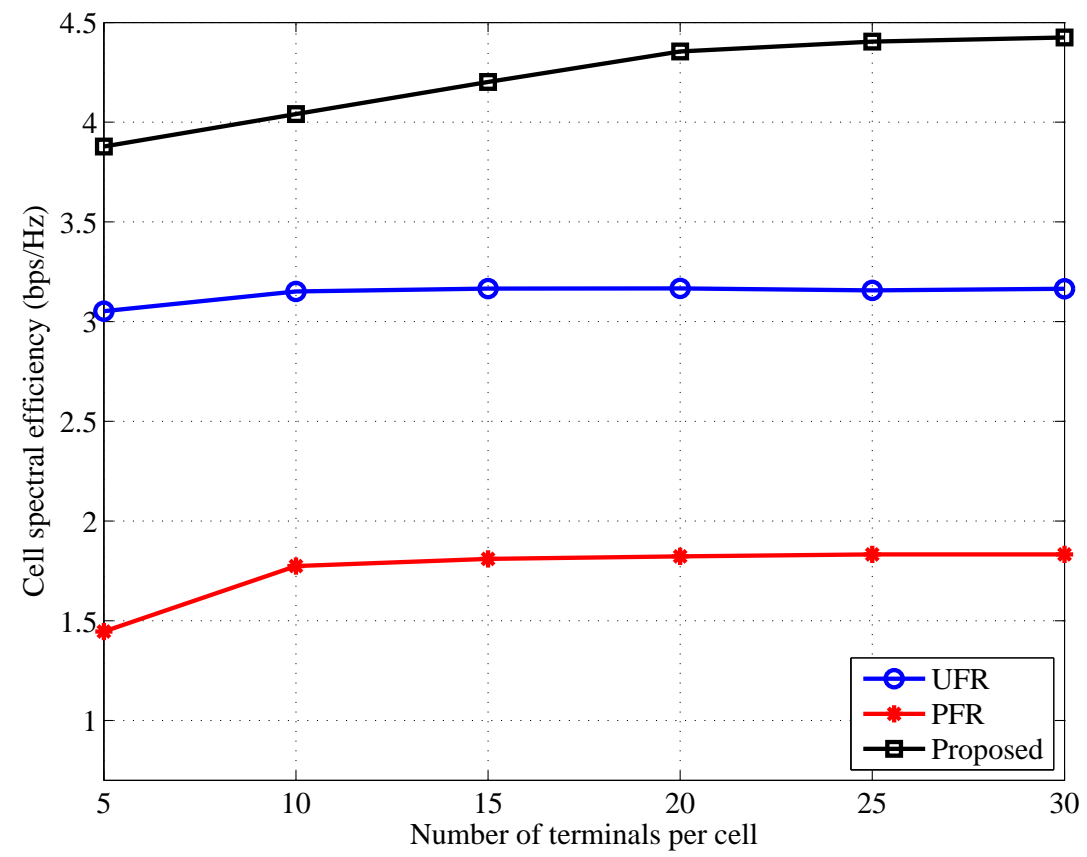

Figure 3.15: Cell spectral efficiency comparison for distribution pattern (c) 


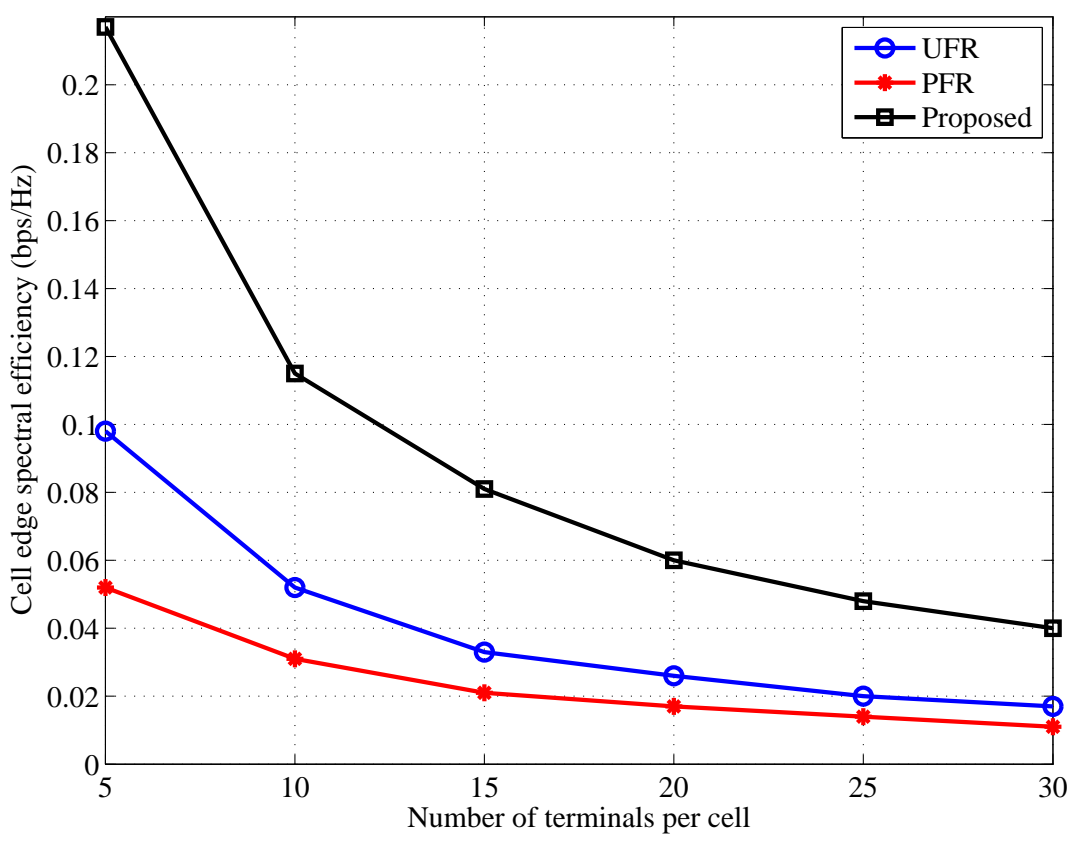

Figure 3.16: Cell edge spectral efficiency comparison for distribution pattern (c) 


\section{Chapter 4}

\section{Autonomous cellular network}

In a typical modern urban cellular network, each BS covers an area with a radius in the range of $100-1000$ meters. By reducing the cell coverage area of each BS, the number of served terminals per BS decreases. Each BS allocates more system resources to each terminal, which in turn results in a larger aggregate throughput for the end-user. The unrelenting increase in the demand for higher data rates requires significant reductions in the cell radii. In a cellular network with a regular deployment of the BSs, this translates into an exceedingly dense infrastructure. The deployment of such an infrastructure would be prohibitively expensive. More importantly, a dense deployment of BSs would not be feasible due to the physical limitations imposed by the urban infrastructure.

Alternatively, a cellular network with small cells can be constructed by the deployment of small BSs (possibly by the end-users) based on the local traffic demand. In this network, the BSs are no longer deployed in a regular fashion. Similar to WiFi networks, the high traffic demand in one location will be reflected in the dense BS deployment in the area. A network with an irregular deployment of the BSs is referred to as an autonomous cellular network.

Let us consider a cellular network with 19 BSs. The network coverage area is modeled as a circle of radius $4.5 R$, where $R$ is the cell radius as illustrated in Figure 4.1. Figure 4.2 
illustrates a traditional network with a regular deployment of the BSs according to the hexagonal pattern on the left and an autonomous cellular network with an irregular deployment of the BSs on the right. The small red circles represent the deployed BSs. Each BS serves a set of terminals. The active terminals have not been shown in this Figure.

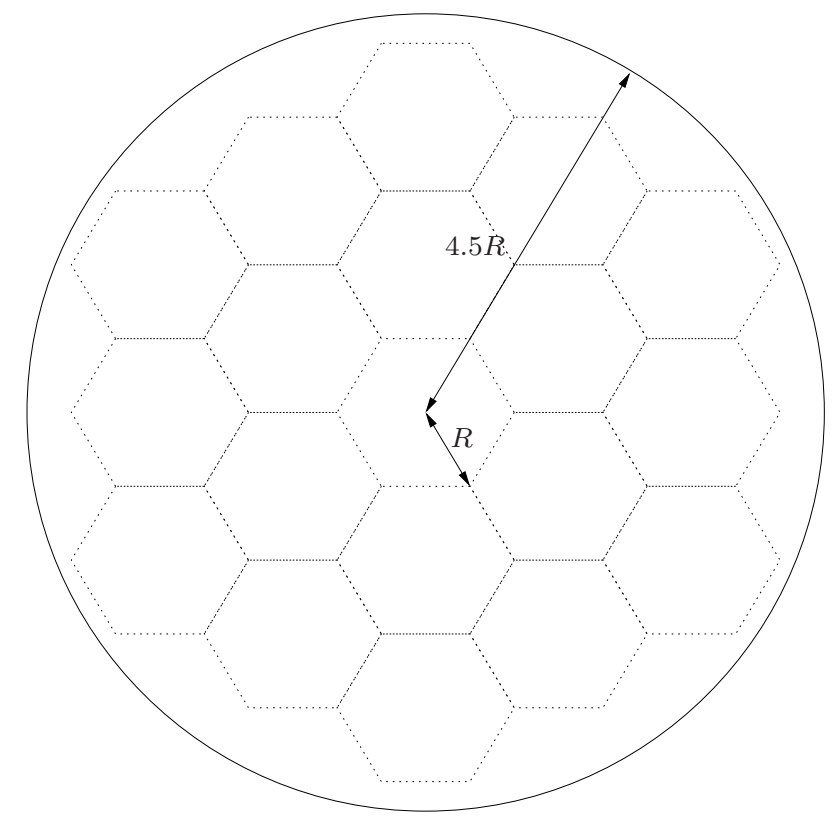

Figure 4.1: Network coverage area for a cellular network with 19 BSs

This chapter is divided into two parts:

- Part I: Let us focus on the two communication links from two BSs to two terminals in an autonomous cellular network as depicted in Figure 4.3. The terminals are shown as blue squares in this Figure. Due to the small distance between the two BSs, it can be safely assumed that the two BSs and the two terminals experience similar topographical properties. In other words, the channel gains of the two links should be correlated. To our knowledge, however, the literature has not reported on the correlation between the channel gains of pairs of links with no common end. This is due to the fact that the models in the literature have been proposed for the traditional cellular network where it is reasonably assumed that the channel gains of 

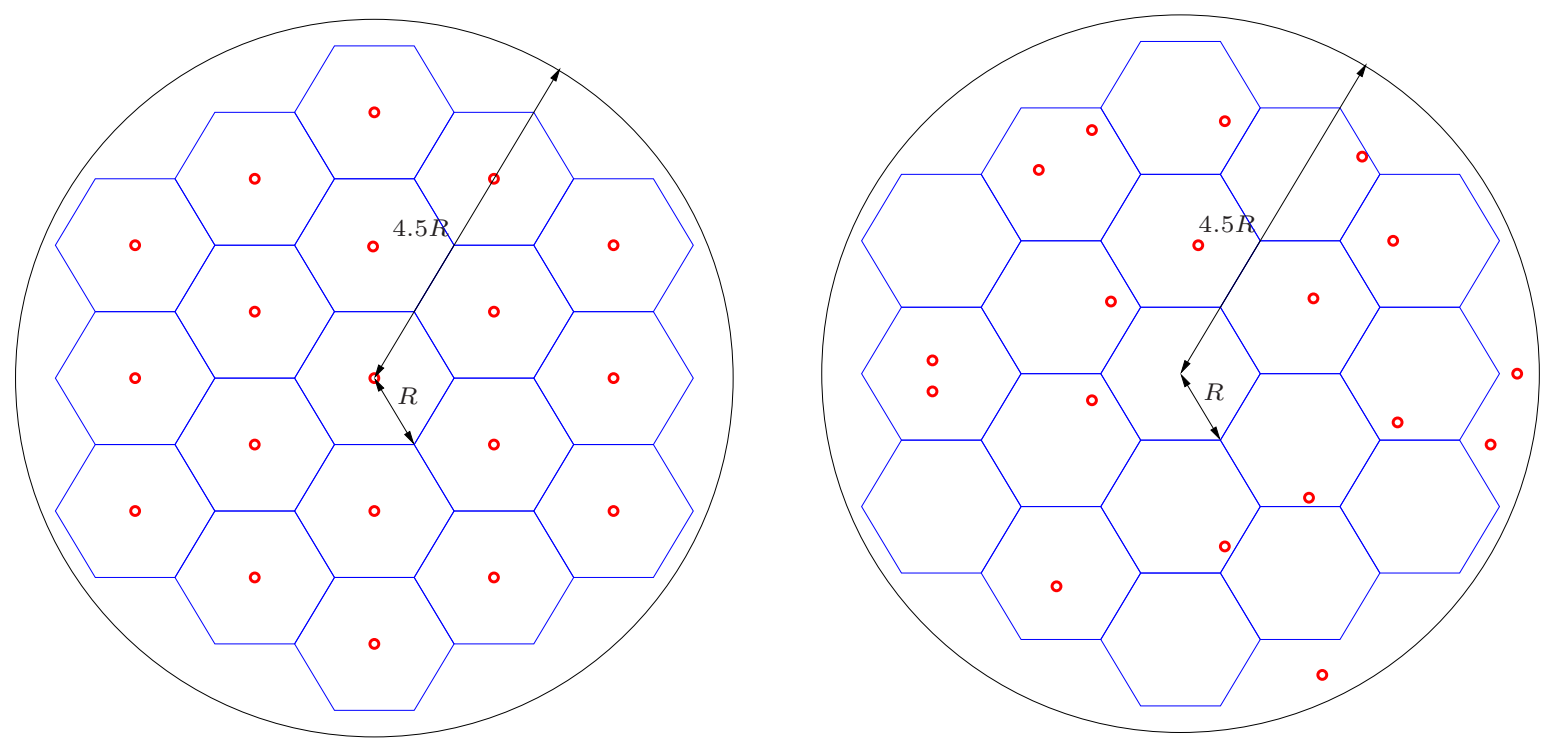

Figure 4.2: Traditional cellular network wit regular BS deployment (left) and autonomous cellular network with irregular BS deployment (right)

pairs of links between terminals and two different BSs are not correlated due to the sufficiently large distances between the BSs. In autonomous cellular networks, on the other hand, it is expected that a large number of BSs will be deployed in random positions. The irregularity of the BS deployment can result in dense clusters within the same locality, i.e. with similar/correlated topographical properties. In this case, the correlation between a pair of links between two terminals and two base stations becomes crucial for a realistic evaluation of the system performance. A network shadow fading model is proposed in this part which introduces correlation between all shadow fading gains across the network based on a common reference.

- Part II: In the literature, resource allocation frameworks have been proposed for the traditional cellular network. In other words, the frameworks have been developed based on the assumption of regular BS deployment. Naturally, they cannot be applied to autonomous networks. In this part a unified framework for resource allocation is developed. The proposed framework does not make any assumption on the deployment pattern of the BSs and hence can be applied to both the traditional 


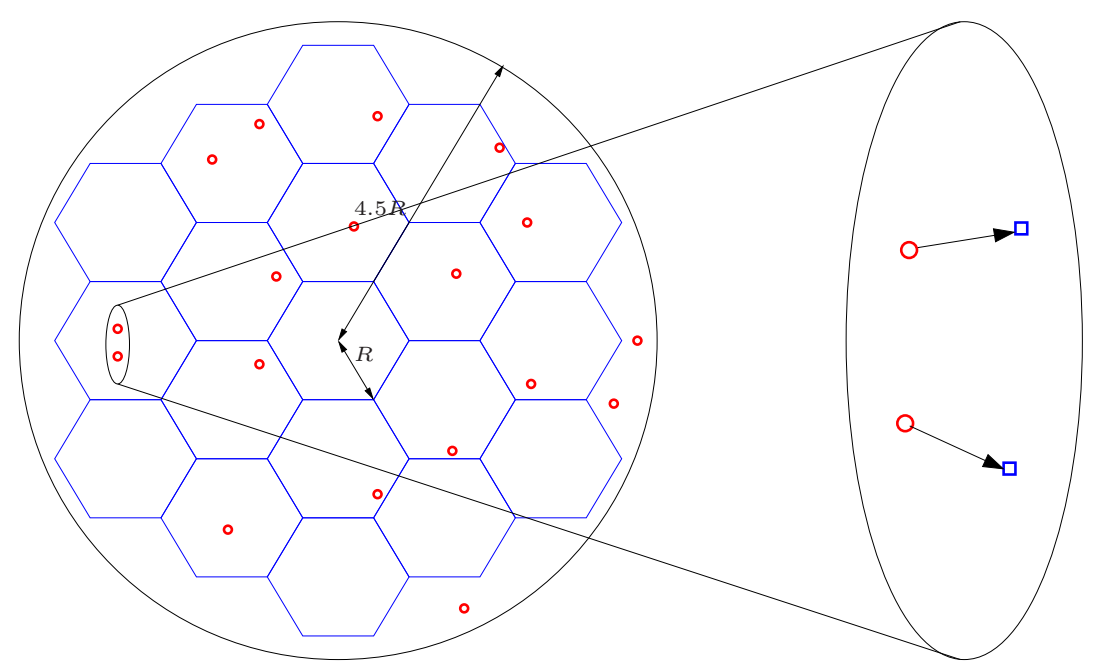

Figure 4.3: Channels gains of two links with no common end should be correlated.

and the autonomous cellular networks.

\subsection{Network shadow fading model}

The propagation of electromagnetic waves in a wireless communication system is studied from two main standpoints. The first deals with the signal power attenuation on a macroscopic level and is often referred to as large-scale (or shadow) fading. The second class considers the fine structure of the multi-path propagation and is known as smallscale (or multi-path) fading. Multi-path fading studies the time and frequency variations of the channel impulse response due to the differences in delays, phases and amplitudes of the multiple reflections of the transmitted signal arriving from different directions at the moving/static receiver. In this section, we exclusively focus on the former class.

At a given distance from the transmitter, large scale fading predicts the average received signal power level over frequency variations and small displacements. The average received power is not only a function of the distance but also highly dependent on the topographical properties of the environment. The physical man made/natural obstructions in the environment should be incorporated into the signal attenuation model. However, 
due to the lack of accurate detailed propagation measurements and landscape information over the large area covered by a typical cellular network — or in order to develop a generic model - the effect of the environment on signal propagation is modeled by introducing a degree of randomness in the average received power level. Based on empirical results, the randomness has been modeled by a log-normal random variable in the signal attenuation model and the effect is referred to as shadow (log-normal) fading.

\subsubsection{Background material}

Consider the link between a transmitter and a receiver. In free space with unobstructed line of sight between the transmit-receive pair, the Friis formula gives the received signal power level, $P_{r}$, as a function of the transmit power level $P_{t}$, the transmitter antenna gain $G_{t}$ and the receiver antenna gain $G_{r}$ as follows:

$$
P_{r}=P_{t} G_{t} G_{r}\left(\frac{\lambda}{4 \pi d}\right)^{2}
$$

where $\lambda$ is the wavelength of the transmitted signal and $d$ is the distance between the transmitter and the receiver.

In terrestrial communication, the received signal power level is a complex function of the topography. To begin with, the rate at which the received power decays with distance is a function of the communication environment (e.g. urban, rural or indoor). On average this rate is modeled with the path-loss exponent $\gamma$. Let us take two links of the same length but sufficiently far from each other in the same environment. Although the distance between the transmitter and the receiver is the same for both, the unique topographical properties cause the received signal powers to be different. Due to the lack of detailed propagation measurements, this effect is statistically modeled by introducing 
a random variable into the equation. Hence the received signal power is given by

$$
P_{r}=K \cdot \frac{1}{d^{\gamma}} \cdot L
$$

where the constant $K$ is a function of the antenna gains, transmission frequency, transmission power and other factors. $L$ is the introduced random variable and is referred to as the shadow fading gain. Based on empirical results, $L$ is modeled as a log-normal random variable, i.e. $\log L \sim \mathcal{N}\left(0, \sigma_{0}^{2}\right)$, where $\mathcal{N}\left(0, \sigma_{0}^{2}\right)$ represents the Gaussian distribution with a mean of zero and a standard deviation of $\sigma_{0}$.

Due to the fact that the topographical properties of the terrain do not change abruptly — the $L$ values for different links are spatially correlated - an appropriate correlation model should be adopted. In 1991, Gudmundson proposed a correlation model [17]. Let us consider the link from point $\mathrm{A}$ to point $\mathrm{B}$ and the link from point $\mathrm{A}$ to point $\mathrm{C}$ in Figure 4.4. The distance between points $\mathrm{B}$ and $\mathrm{C}$ is denoted by $d$. The correlation between the links $\mathrm{A}-\mathrm{B}$ and $\mathrm{A}-\mathrm{C}$ is a function of $d$ as follows:

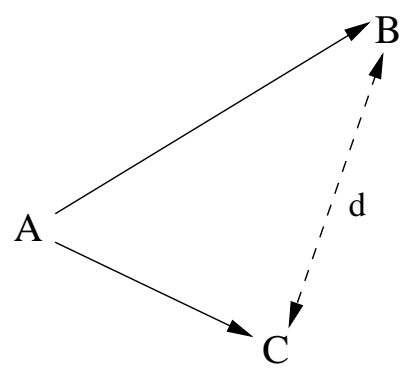

Figure 4.4: Two links from a common point to two points apart by a distance of $d$

$$
R(d)=\sigma_{0}^{2} e^{-d / d_{c}}
$$

where $d_{c}$ is the correlation distance. According to the Gudmundson model, the channel gains between a given BS and a set of $K$ terminals are generated in the following four steps: 
- Step 1: Generate a $K \times 1$ vector $\mathbf{v}$, where $v_{i}$ 's are independent Gaussian random variables with a standard deviation of 1 .

- Step 2: Generate the $K \times K$ correlation matrix $\mathbf{R}=\left[r_{i j}\right]$ such that,

$$
r_{i j}=\sigma_{0}^{2} \exp \left(-d_{i j} / d_{c}\right)
$$

where $d_{i j}$ denotes the distance between terminal $i$ and terminal $j$.

- Step 3: Decompose the correlation matrix using the Cholesky factorization such that $\mathbf{R}=\mathbf{B B}^{H}$.

- Step 4: The correlated channel gains between the BS and the $K$ terminals are stacked in the $K \times 1$ vector $\mathbf{B v}$.

In [25] a two-dimensional spatial correlation model is proposed. The model generates the correlated shadow fading gains between a BS and multiple terminals. In order to do so, for each BS, a two-dimensional Gaussian random field with appropriate marginal distribution and spatial correlation is generated ${ }^{1}$. A Gaussian field is a random process where each realization is a function from the plane to the real numbers. If we fix a point in the plane, the outcome is a Gaussian random variable. The shadow fading gain between the BS and any terminal in the field is the value of this random variable at the terminal.

We propose a unified approach to generate correlated shadow fading gains between any two links in the network. In particular, consider the following two links A-B and C-D (see Figure 4.5). The two transmitters A and C are located close to each other. Similarly receivers B and D are also located close to one another. Clearly the shadow fading gains of the two links should be correlated. However, since the two links do not

\footnotetext{
${ }^{1}$ The underlying spatial correlations are generated based on the Gudmundson model.
} 
share a common end, the Gudmundson correlation model would not introduce correlation between the shadow fading gains for these two links.

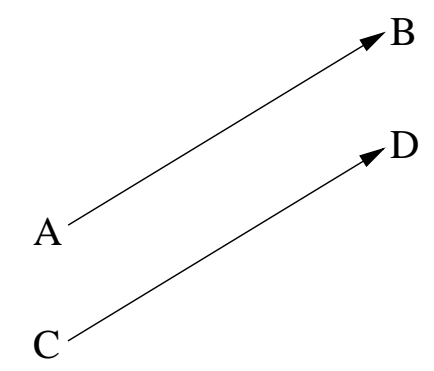

Figure 4.5: A pair of links with the same topographical properties

\subsubsection{Network correlation model}

In this section we develop a generalized model for shadow fading in cellular networks. The key idea is to develop a common reference based on which all shadow fading gains are generated. With such a reference, all channel gains in the network become correlated whether they share a common end or not. This underlying universal basis is referred to as the potential field. The value of this field at point $\mathrm{A}$ is referred to as the potential level of point $\mathrm{A}$ and is denoted by $X_{A}$.

Mathematically, the potential field is defined as a correlated two-dimensional Gaussian field across the network coverage area. In a cellular network with $A$ BSs and $K$ terminals, $N=K+A$ potential levels are generated in the following four steps:

- Step 1: Generate an $N \times 1$ vector $\mathbf{v}$, where $v_{i}$ 's are independent Gaussian random variables with a standard deviation of 1 .

- Step 2: Generate the $N \times N$ correlation matrix $\mathbf{R}=\left[r_{i j}\right]$ such that,

$$
r_{i j}=\sigma_{X}^{2} \exp \left(-d_{i j} / d_{c}\right)
$$


where $d_{i j}$ denotes the distance between point $i$ and point $j$ and the appropriate value for $\sigma_{X}$ is discussed later in this section.

- Step 3: Decompose the correlation matrix using the Cholesky factorization such that $\mathbf{R}=\mathbf{B B}^{H}$.

- Step 4: The potential levels of the $N$ points are stacked in the $N \times 1$ vector $\mathbf{B v}$.

The shadow fading gain between any two points is generated by a function of the potential levels of the two ends of the corresponding link. Before introducing this function, let us study the properties of the shadow fading gain in more detail.

The shadow fading gain of a link between point $\mathrm{A}$ and point $\mathrm{B}$ should have the following three properties:

- Symmetry: The channel gain is intrinsically symmetric. Regardless of which end the transmitter (or the receiver) lies, the channel gain should be the same.

- Continuity: As the two ends of a link come closer to each other, the randomness due to the topography of the environment diminishes. This should translate into a smaller variance for the shadow fading gain.

- Compatibility: Based on empirical results, the shadow fading gain in the logarithmic scale $(\mathrm{dB})$ is modeled as a zero-mean Gaussian random variable with a standard deviation of $\sigma_{0} \mathrm{~dB}$.

The following function is proposed for the shadow fading gain (in dB) between point A and point B:

$$
\mathfrak{f}\left(X_{A}, X_{B}\right)=\operatorname{sgn}\left(X_{A}+X_{B}\right) \cdot\left|X_{A}-X_{B}\right|
$$

where, $\operatorname{sgn}(t)$ is the signum function. The proposed function satisfies the symmetry property. The continuity and the compatibility properties are verified by the following lemma. 
LEmma: If $S$ and $T$ are two independent Gaussian random variables with zero mean and standard deviations of $\sigma_{S}$ and $\sigma_{T}$ respectively, the random variable $Z=\operatorname{sgn}(S) \cdot|T|$ has a Gaussian distribution with zero mean and a standard deviation of $\sigma_{T}$.

Proof: Since $X$ is a zero-mean Gaussian random variable, $t=\operatorname{sgn}(X)$ is a discrete random variable with $P(t=1)=P(t=-1)=0.5$. The cumulative distribution function of $Z$ is

$$
F_{Z}(z)=P(Z \leq z)=0.5 P(|T| \leq z)+0.5 P(-|T| \leq z)
$$

or,

$$
F_{Z}(z)= \begin{cases}\frac{1}{2} P(|T| \leq z)+\frac{1}{2} & , z \geq 0 \\ \frac{1}{2} P(-|T| \leq z) & , z<0\end{cases}
$$

It is well-known that the random variable $L=|T|$ has a half-normal distribution with probability distribution function

$$
f_{L}(l)=\sqrt{\frac{2}{\pi \sigma_{T}^{2}}} \exp \left(-\frac{l^{2}}{2 \sigma_{T}^{2}}\right)
$$

Hence when $z \geq 0$,

$$
\begin{aligned}
F_{Z}(z) & =\frac{1}{2} P(L \leq z)+\frac{1}{2} \\
& =\frac{1}{2}\left(1+\sqrt{\frac{2}{\pi \sigma_{T}^{2}}} \int_{0}^{z} \exp \left(-\frac{l^{2}}{2 \sigma_{T}^{2}}\right) d l\right) \\
& =\frac{1}{2}\left(2-\sqrt{\frac{2}{\pi \sigma_{T}^{2}}} \int_{z}^{+\infty} \exp \left(-\frac{l^{2}}{2 \sigma_{T}^{2}}\right) d l\right) \\
& =1-\frac{1}{\sqrt{2 \pi \sigma_{T}^{2}}} \int_{z}^{+\infty} \exp \left(-\frac{l^{2}}{2 \sigma_{T}^{2}}\right) d l \\
& =\frac{1}{\sqrt{2 \pi \sigma_{T}^{2}}} \int_{-\infty}^{z} \exp \left(-\frac{l^{2}}{2 \sigma_{T}^{2}}\right) d l
\end{aligned}
$$


and for $z<0$,

$$
\begin{aligned}
F_{Z}(z) & =\frac{1}{2} P(-L \leq z) \\
& =\frac{1}{2} P(L \geq-z) \\
& =\frac{1}{\sqrt{2 \pi \sigma_{T}^{2}}} \int_{-z}^{+\infty} \exp \left(-\frac{l^{2}}{2 \sigma_{T}^{2}}\right) d l \\
& =\frac{1}{\sqrt{2 \pi \sigma_{T}^{2}}} \int_{-\infty}^{z} \exp \left(-\frac{l^{2}}{2 \sigma_{T}^{2}}\right) d l
\end{aligned}
$$

Hence, $Z \sim \mathcal{N}\left(0, \sigma_{T}^{2}\right)$.

Let $S$ and $T$ take the values of $X_{A}+X_{B}$ and $X_{A}-X_{B}$ respectively. The two random variable are uncorrelated since

$$
\mathrm{E}\{S T\}=\mathrm{E}\left\{\left(X_{A}+X_{B}\right)\left(X_{A}-X_{B}\right)\right\}=0
$$

Hence $S$ and $T$ are independent as they are both zero mean Gaussian random variables. Based on this lemma, the shadow fading gain $\mathfrak{f}\left(X_{A}, X_{B}\right)$ is Gaussian with variance

$$
\sigma^{2}=\mathrm{E}\left\{\left(X_{A}-X_{B}\right)^{2}\right\}=2 \sigma_{X}^{2}-2 \mathrm{E}\left\{X_{A} X_{B}\right\}=2 \sigma_{X}^{2}\left(1-e^{-d_{A B} / d_{c}}\right)
$$

where $d_{A B}$ is the distance between points $\mathrm{A}$ and $\mathrm{B}$ and $\sigma_{X}^{2}$ is the variance of the potential levels $X_{A}$ and $X_{B}$. It is clear that the variance of the shadow fading gain approaches zero as $d_{A B} \rightarrow 0$. Conversely, with $d_{A B} \rightarrow \infty$, the variance of the shadow fading gain converges to $2 \sigma_{X}^{2}$. This value should match the variance of the Gudmundson model, i.e. $\sigma_{0}^{2}$. Hence, the potential field is generated with a variance of $\sigma_{X}^{2}=\frac{1}{2} \sigma_{0}^{2}$. Thus,

$$
\sigma^{2}=\sigma_{0}^{2}\left(1-e^{-d_{A B} / d_{c}}\right)
$$


Figure 4.6 illustrates a realization of the potential field in an area of $300 \times 300 \mathrm{~m}^{2}$. The potential levels are generated for $60 \times 60$ points located on a grid where each two adjacent points are 5 meters apart with $\sigma_{X}=4$.

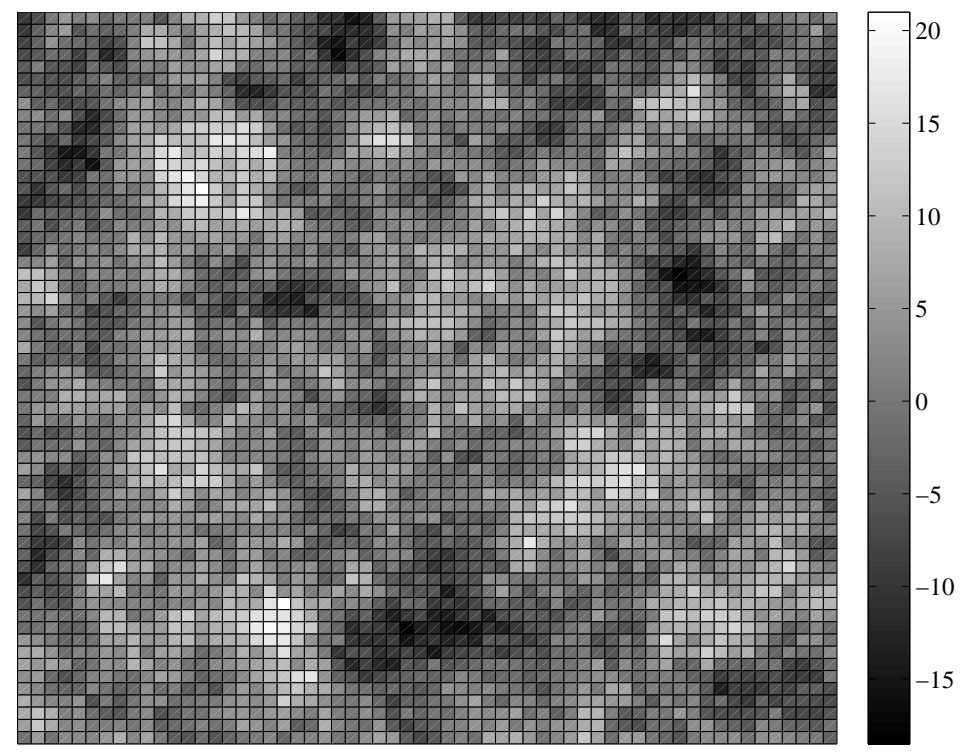

Figure 4.6: A realization of the network potential field

As the two points A and B come closer to each other, the correlation between their corresponding potential levels increases. Hence, the variance of the shadow fading gain approaches zero which satisfies the continuity property. Figure 4.7 depicts the normalized variance $\left(\sigma^{2} / \sigma_{0}^{2}\right)$ as a function of the normalized distance $\left(d / d_{c}\right)$. It is observed that when the distance between the transmitter and receiver increases to more than 5 times the correlation distance, the variance matches that of the Gudmundson model, i.e. $\sigma_{0}^{2}$.

It is important to note that the variance of the Gudmundson model is not a function of distance. This violates the continuity property at $d$ equal to zero. By introducing the underlying potential field, we are able to maintain this important property in the network shadow fading model. 


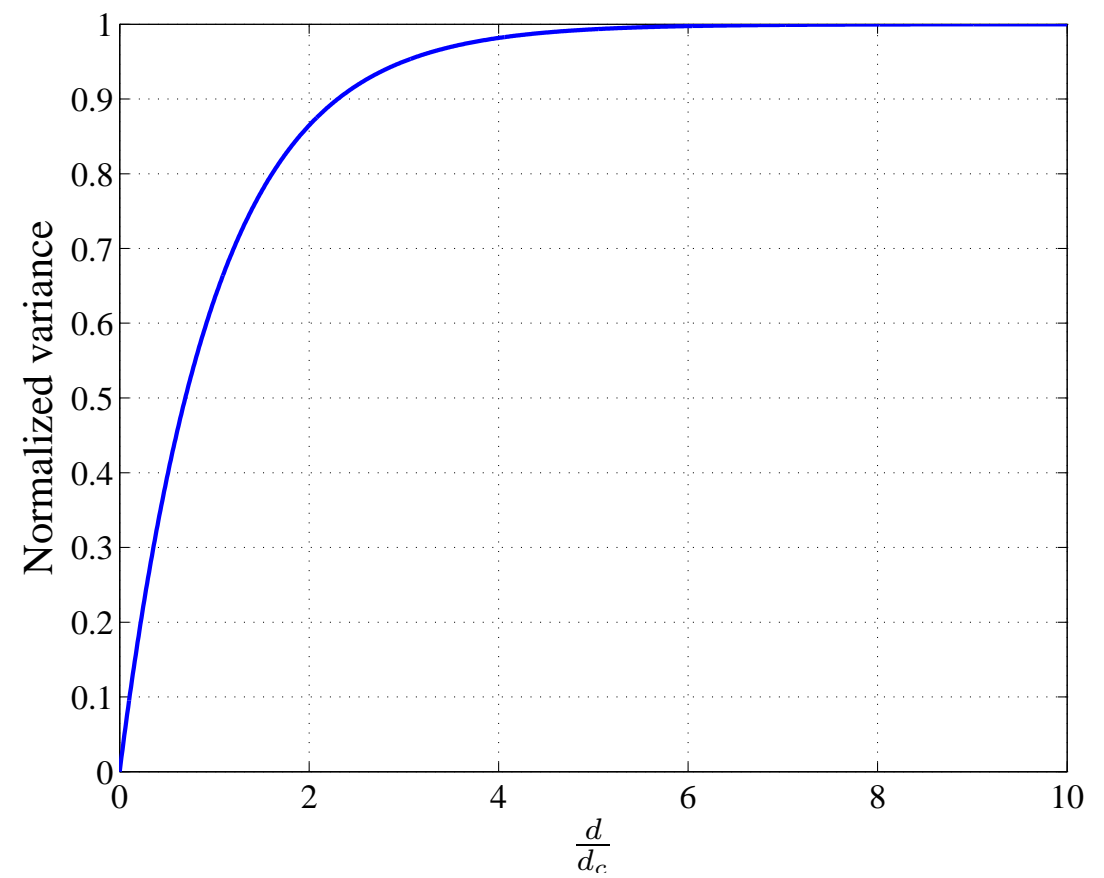

Figure 4.7: Normalized variance of the shadow fading gain

\subsubsection{Cell radius}

In a cellular network each terminal is assigned to the BS with the strongest channel gain. Each BS serves a set of terminals. The set of terminals together with the serving BS form a cell. The cell is often viewed as a circle with the BS at the center and a radius which delineates the coverage area of the BS. Although the set of terminals served by a BS are clearly defined, the radius of a cell is not a well-defined parameter. In this section we introduce the notion of the outage radius for a cell.

The outage probability for a link in a wireless network is defined as follows:

$$
P_{\text {out }}(d)=\operatorname{Prob}\left(P_{r} \leq P_{\text {min }}\right)=\operatorname{Prob}\left(\frac{K}{d^{\gamma}} \cdot L \leq P_{\text {min }}\right)
$$

where, $K$ is directly proportional to the transmit power of the BS and $P_{\min }$ is the minimum required received power.

Let us define the cell outage radius $R$ as the distance (from the BS) where the outage 
probability is equal to $5 \%$. By setting the outage probability to 0.05 and solving for $R$, the cell outage radius (in meters) is calculated as

$$
P_{\text {out }}(R)=0.05 \Rightarrow R=\left(\frac{K}{P_{\min }} 10^{-\frac{\sigma \sqrt{2}}{10} \operatorname{erfc}^{-1}(0.1)}\right)^{\frac{1}{\gamma}}
$$

where $\operatorname{erfc}($.$) is the complementary error function. By increasing the transmit power K$, the cell outage radius grows and hence a larger cell is formed. In a sense, this definition maps the transmission level of a BS into a geographical distance in meters which can be viewed as the cell radius.

In (4.3) the correlation distance $d_{c}$ is defined as the distance between two points B and $\mathrm{C}$ (see Figure 4.4) whose channel gains have a correlation of $R\left(d_{c}\right)=\sigma_{0}^{2} e^{-1}$. The value of the correlation distance depends on the intrinsic properties of the environment. A large correlation distance suggests an environment with a small number of scatterers in a possibly open rural area. Conversely, a small correlation distance translates into an urban environment with a dense infrastructure. In the analysis of the network, the cell radius in meters would not provide much useful information about the cell. In a rural environment (with a large correlation distance) a cell with a radius of 100 meters may be considered as a small cell. Meanwhile, the same cell in an urban environment (with a small correlation distance) may be considered as a large one. As a result, in the analysis of the proposed model all distances are normalized by the correlation distance $d_{c}$. The large and small cells are modeled with cells of radii $100 d_{c}$ and $d_{c}$ respectively.

\subsubsection{Outage probability}

Using (4.14), the outage probability can be written as a function of the cell outage radius as follows: 


$$
\begin{aligned}
P_{\text {out }}(d) & =\operatorname{Prob}\left(\frac{K}{d^{\gamma}} \cdot L \leq P_{\min }\right) \\
& =\operatorname{Prob}\left[L \leq\left(\frac{d}{R}\right)^{\gamma} \cdot 10^{\left.-\frac{\sigma \sqrt{2}}{10} \operatorname{erfc}^{-1}(0.1)\right]}\right. \\
& =\frac{1}{2} \operatorname{erfc}\left(-\frac{10 \log _{10}\left(\frac{d}{R}\right)^{\gamma}-\sigma \sqrt{2} \operatorname{erfc}^{-1}(0.1)}{\sigma \sqrt{2}}\right)
\end{aligned}
$$

In Figure 4.8 the outage probability of a cell with an outage radius of $100 d_{c}$ (large cell) is plotted as a function of the normalized distance $\left(d / d_{c}\right)$. The outage probability according to the Gudmundson model is also provided for comparison. The figure shows that both models predict the same outage probability for a large cell. Figure 4.9 depicts the outage probability for a cell with an outage radius of $d_{c}$ (small radius). Unlike the large cell, the outage probability of the proposed model is lower than that of the Gudmundson model for a small cell. This behavior can be explained by the smaller variance of the shadow fading gain for short distances.

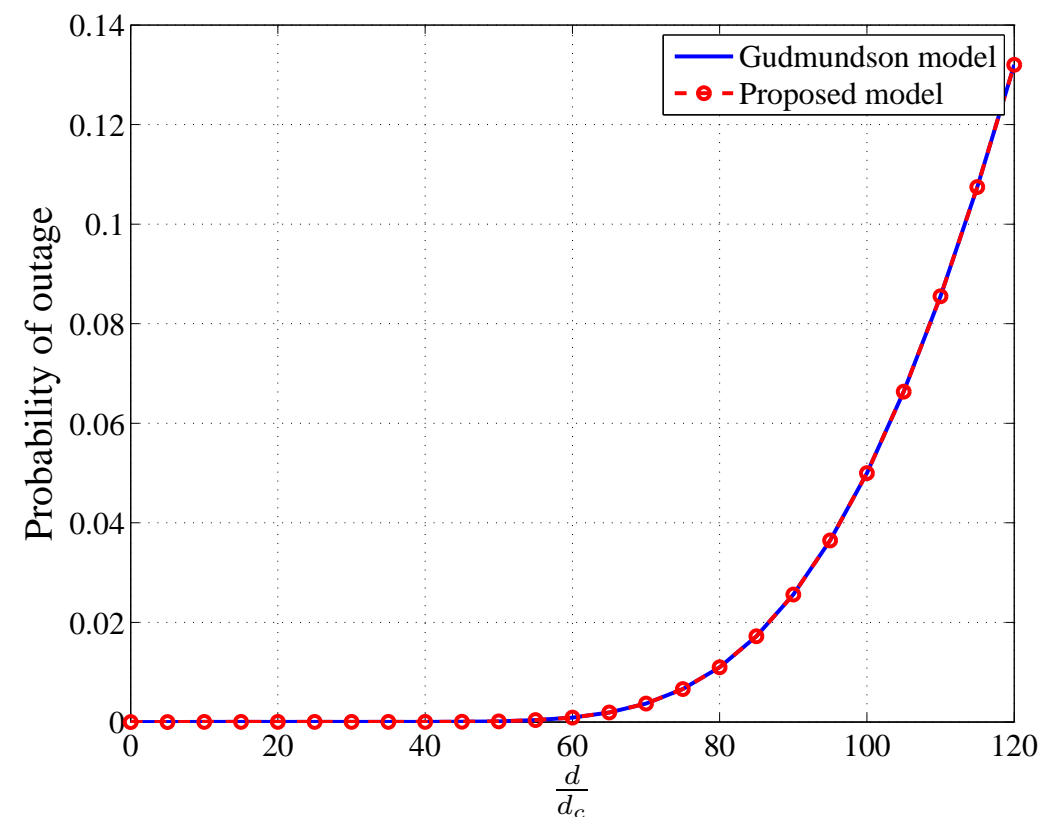

Figure 4.8: Outage probability for large cells $\left(R=100 d_{c}\right)$ vs. the normalized distance for the Gudmundson and the proposed models 


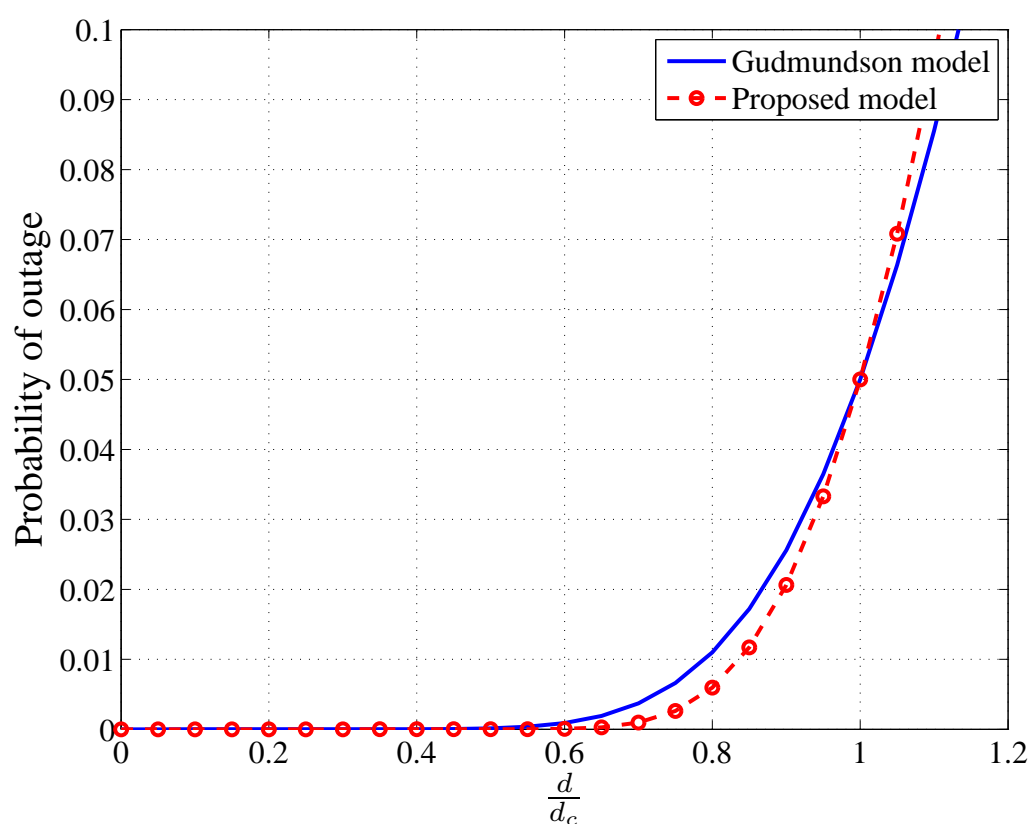

Figure 4.9: Outage probability for small cells $\left(R=d_{c}\right)$ vs. the normalized distance for the Gudmundson and the proposed models

\subsubsection{Correlation between two links with a common end}

The proposed model provides a unified approach to generate correlated channel gains between all links in the network. The Gudmundson model, however, generates correlation between the channel gains only when the two links share a common end. In order to compare the correlation properties of the two models, we consider this special case. Let the two terminals $k_{1}$ and $k_{2}$ be located at a distance $D \gg d_{c}$ from a BS as illustrated in Figure 4.10. By varying the angle $\theta$, the distance $d$ between the terminals changes and is bounded between 0 (with $\theta=0$ ) and $2 D$ (with $\theta=\pi$ ).

Figure 4.11 depicts the normalized correlation function $R(d) / \sigma_{0}^{2}$ versus distance $d$ for the proposed and the Gudmundson models. As the distance between the two terminals increases, the Gudmundson model predicts a decreasing correlation between the two channel gains which converges to zero. The proposed model, on the other hand, predicts a correlation which decreases and converges to a non-zero constant value. This phenomenon 


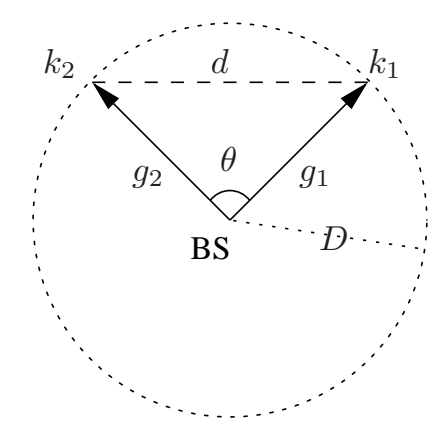

Figure 4.10: Common end simulation model

can be explained as follows:

The correlation between the shadow fading gains $g_{1}$ and $g_{2}$ depends on the topographical properties along the path of communication of the two links. Let us consider the case where the BS is located on top of an elevation. Due to the location of the BS, with high probability there exists a Line-Of-Sight (LOS) path between the BS and the two terminals, which in turn translates into relatively high channel gains on both links. On the other hand, if the BS is located in a vicinity with many obstructions (e.g. in the middle of tall buildings), regardless of the distance of the terminals $d$, both terminals would experience relatively weak channel gains from the BS. Based on these arguments, it is clear that the channel gains $g_{1}$ and $g_{2}$ should be correlated even when the distance between the two terminals $d$ is large.

In the rest of this Chapter the proposed resource allocation framework for the autonomous cellular networks is presented.

\subsection{Network clustering}

A scheduling cell is formed by a set of BSs whose resource allocation is coordinated. In Chapter 3, scheduling cells were introduced in the traditional cellular network. Based on the pre-determined regular locations of the BSs, scheduling cells were formed which were static, regular and equal in size. Although the proposed resource allocation framework 


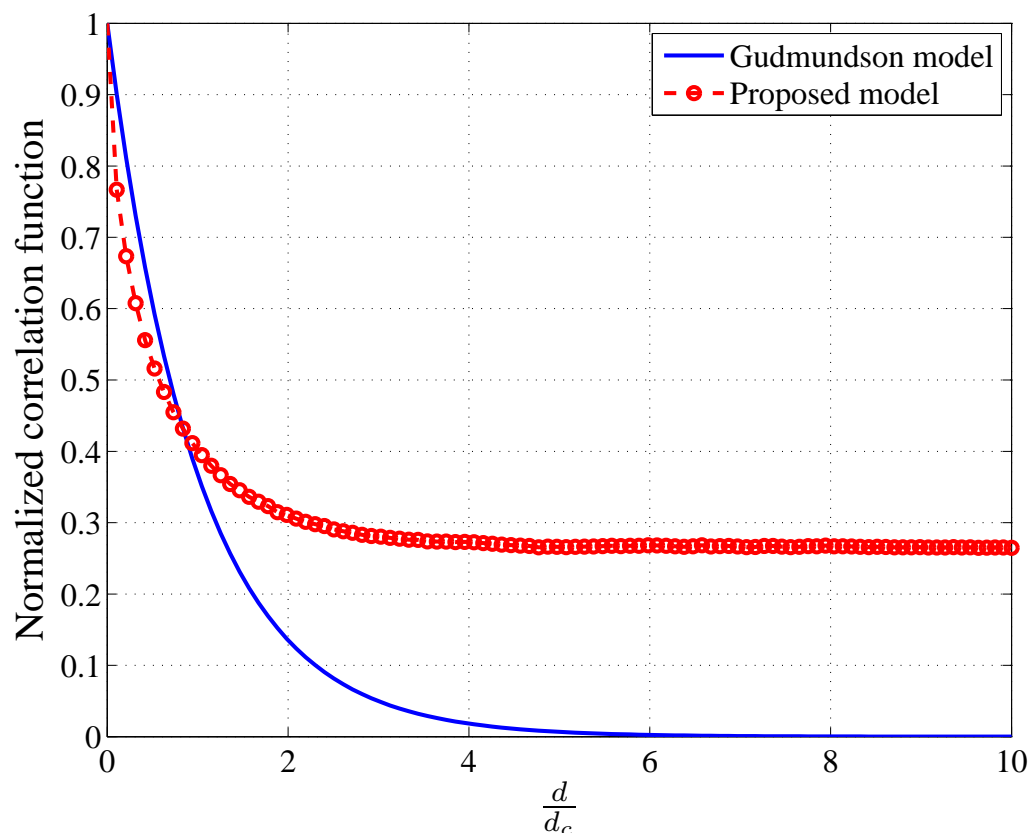

Figure 4.11: Normalized correlation function vs. the normalized distance for the Gudmundson and the proposed models

adapted to the local traffic patterns, it relied heavily on the network structure, i.e. the location of the BSs. In this chapter, adaptive scheduling cells are developed. The cells adapt to the actual traffic distribution across the network. They are no longer required to be of the same size. In regions where high interference between BSs call for more coordination (e.g. hot-spots), a larger cluster of BSs form a scheduling cell. Conversely, regions which serve sparse population of terminals and/or the terrain obstructs interference between BSs, smaller scheduling cells are created. A coordinated resource allocation method tailored to the irregular structure of the adaptive scheduling cells is proposed. The corresponding resource allocation optimization problem was formulated in [33] and a solution was proposed. This solution is modified according to our assumptions, requirements and objectives.

The performance of the proposed resource allocation framework is evaluated for the traditional and the autonomous cellular networks. In the traditional network, the BSs are assumed to be of the same size and power budget and the BS deployment follows 
the regular hexagonal pattern. An autonomous cellular network, on the other hand, is formed by an irregular deployment of the BSs.

\subsubsection{Problem definition}

In order to provide service to a set of $K$ wireless terminals, a set of $A$ BSs are deployed in the network coverage area. Each BS has a backbone connection to the wired network and is equipped with a single antenna. We assume that each terminal is assigned to the BS with the strongest channel gain. The communication between the BSs and the terminals occurs on the available system spectrum, a set of $N$ frequency resources.

The resource allocation problem has been formulated in Section 2.1. The optimal solution would require full coordination between all BSs . However, full coordination between all BSs in the network is prohibitively expensive and hence not practical. Alternatively, the resource allocation problem can be broken down into smaller subproblems and solved locally. Instead of coordinating the resource allocation among all BSs, the coordination can be between a subset (cluster) of BSs. It is assumed that the entire frequency spectrum is available to all clusters. Hence, there would inevitably exist a level of inter-cluster interference between any two clusters of BSs. At the time of resource allocation for each time slot, the coordinating BSs adjust their transmission power levels to achieve the best performance. The BSs outside the cluster, however, induce interference on all links in the cluster and degrade the performance. Naturally, larger clusters of BSs would result in a better performance due to the lower number of potentially interfering BSs outside of each cluster. However, the improvement in the performance comes at an extra cost of higher complexity for coordination between a larger number of BSs. This chapter, develops a framework where clusters of coordinating BSs are formed adaptively based on the locations of the BSs and the traffic distribution in the network. The size of the clusters can be adjusted according to the desired trade-off between complexity and performance. 
Similar to Chapter 3, we define a scheduling cell (SC) as a collection of BSs with coordinated resource allocation. At one extreme, one can define the entire network to be one SC. In this case a fully coordinated resource allocation would result in optimal performance. Nevertheless, the resource allocation would be extremely complex to implement. At the other extreme, if we defined each BS to be one SC, the level of frequency reuse could result in unacceptable interference levels between the scheduling cells.

Let us define the topology of the network by the interference matrix $\mathbf{H}=\left[h_{l i}\right] \in \mathbb{R}^{K \times A}$, where $h_{l i}$ denotes the channel gain between terminal $l$ and BS $i$. High channel gains between terminals and BSs result in dense topologies and call for coordination in resource allocation. On the other hand, a sparse topology is formed as a result of low channel gains between terminals and the interfering BSs. Naturally, the size of a scheduling cell ${ }^{2}$ should depend on the topology of the network. Although a large scheduling cell implies high coordination in resource allocation and hence a better system performance, it in turn increases the implementation cost and complexity. On the other hand, too small a scheduling cell results in excessive interference levels between the scheduling cells.

Definition 4.1 (Network clustering problem) Let $\mathcal{A}$ be the set of the BSs in the network. A partition of $\mathcal{A}$ is defined as the set of subsets $\{\mathcal{A}(1), \mathcal{A}(2), \cdots, \mathcal{A}(C)\}$ such that

$$
\bigcup_{c=1}^{C} \mathcal{A}(c)=\mathcal{A} \quad \text { and } \quad \mathcal{A}(i) \cap \mathcal{A}(j)=\emptyset, \quad \forall i \neq j
$$

Clustering is the process of finding such a partition subject to some criteria. Resource allocation in each scheduling cell is both coordinated and autonomous. Coordinated resource allocation in each scheduling cell increases the efficiency of system resource utilization. Autonomy implies the independence of resource allocation in a scheduling cell from the rest of the network. It should be noted that this does not mean that the achieved performance of the scheduling cells are independent (or the scheduling cells are

\footnotetext{
${ }^{2}$ The size of a scheduling cell is defined as the number of BSs belonging to the scheduling cell.
} 
completely isolated). In fact, the interference from other scheduling cells impacts the performance of any given scheduling cell.

The clustering problem imposes boundaries between the scheduling cells. Each boundary delineates the lack of coordination between certain BSs. This in turn means that unlike the traditional frequency allocation schemes the transmission to the terminals at the boundary between two adjacent scheduling cells will no longer be perfectly orthogonal. It should be noted, however, that the level of interaction between scheduling cells heavily depends on the network topology. An intelligent choice of the scheduling cells' boundaries reduces the inter-cell interference to acceptable levels. More importantly, such a clustering of the network makes the introduction of practical coordinated resource allocation methods in cellular networks possible.

Let us model the cellular network as a fully connected weighted graph $\mathcal{G}=(\mathcal{V}, \mathcal{E})$. The set of vertices $\mathcal{V}$ represents the set of BSs $\mathcal{A}$. Each edge connects two BSs in the network. The weight on the edge is defined based on the notion of interference between BSs. This notion is introduced in Section 4.2.2 which gives rise to the definition of the similarity index. Subsequently in Section 4.2.3, a clustering algorithm is proposed which partitions the BSs into scheduling cells.

\subsubsection{Similarity index}

Resource allocation in a cellular network is performed in a time-slotted fashion. At each time slot, each BS serves a number of terminals on the available frequency channels. In order to avoid excessive interference, each BS schedules only one terminal on each frequency channel. It is possible, however, to assign more than one frequency channel to a terminal in a time slot. The SINR of a terminal operating on a frequency channel can be written as:

$$
\mathrm{SINR}=\frac{P_{D}}{P_{I}+P_{N}}
$$


where $P_{D}$ is the received signal from the desired BS at the desired terminal. $P_{I}$ denotes the interference at the desired terminal and $P_{N}$ is the background noise level. The interference emanates from the simultaneous transmission (i.e. at the same time slot and on the same frequency channel) from all or a subset of the BSs in the network.

The quality of the communication between a terminal and a BS can be quantified in many ways (supported data rate, bit error rate, ...). Regardless of the definition, the quality improves with a higher level of SINR. Let us define the performance level of a terminal by the corresponding SINR level. Each BS serves a set of terminals with a unique set of performance levels. Consider two BSs $i$ and $j$. The simultaneous transmission from BS $j$ has an impact on the performance levels of the terminals assigned to BS $i$. If the impact is significant, coordination in resource allocation between the two BSs is required.

Let us define the performance level of BS $i$ by an increasing function of the performance levels of the terminals assigned to this BS. If the simultaneous transmission from BS $j$ significantly impacts the performance level of BS $i$ (or if the simultaneous transmission from BS $i$ significantly impacts the performance level of BS $j$ ), coordination is required for resource allocation between the two BSs. The performance level of BS $i$ can be defined as the arithmetic average of the performance levels of the terminals assigned to BS $i$. This definition, however, has one major shortcoming. The SINR levels of the terminals assigned to a BS can take a wide range of values. In some scenarios, due to the exceptionally good channel gains (or unrealistic simulation models or flaws in channel estimations), a few terminals experience unexpectedly high SINR levels. The resource allocation decisions, however, should not be influenced by or be based on the channel gains of these terminals. Albeit, the arithmetic average SINR level is skewed toward these high values. The median SINR level, on the other hand, is defined as the midpoint where half of the SINR levels are above and half of the SINR levels are below this value. Essentially, by defining the performance level of a BS as the median value, the effect of the unrealistically high SINR levels is mitigated. It should be noted that this definition 
is not unique and the BS performance level may also be defined in other ways.

Based on the interference coming from $\mathrm{BS} j$, the signal-to-interference-plus-noise ratio (SINR) at terminal $l$ assigned to BS $i$ can be written as

$$
\operatorname{SINR}_{l i j}=\frac{h_{l i} p_{i}}{h_{l j} p_{j}+\eta_{0} W}
$$

where $h_{l i}$ denotes the channel gain between terminal $l$ and $\mathrm{BS} i, p_{i}$ is the transmit power of $\mathrm{BS} i$ and $\eta_{0} W$ denotes the background noise power level. With no interference from $\mathrm{BS} j$, the signal-to-noise ratio (SNR) for terminal $l$ is

$$
\mathrm{SNR}_{l i}=\frac{h_{l i} p_{i}}{\eta_{0} W}
$$

Let us form the SINR vector $\mathbf{x}_{i j}$ by the SINR levels of all terminals assigned to BS $i$ with interference from BS $j$ as calculated in (4.18). Similarly, let $\mathbf{y}_{i}$ be the vector of the $\mathrm{SNR}_{l i}$ levels of the terminals assigned to $\mathrm{BS} i$ as calculated in (4.19). The loss in performance due to the effect of interference from $\mathrm{BS} j$ on $\mathrm{BS} i$ is quantified by the following index:

$$
c_{i j}=\frac{f\left(\mathbf{y}_{i}\right)}{f\left(\mathbf{x}_{i j}\right)}
$$

where, $f\left(\mathbf{y}_{i}\right)$ is the performance level of BS $i$ without interference from BS $j$. Similarly, $f\left(\mathbf{x}_{i j}\right)$ is the performance level of $\mathrm{BS} i$ with interference from $\mathrm{BS} j$. Based on the discussions earlier in this section, the performance level of each BS is defined as the median of the performance levels of all terminals assigned to the BS.

By exchanging $i$ and $j$ in (4.18), (4.19) and (4.20) $c_{j i}$ is defined. A larger $c_{i j}$ and/or $c_{j i}$ implies higher levels of interference between BSs $i$ and $j$, and in turn translates into a higher urgency for coordination between the two BSs. The similarity index between BS 
$i$ and BS $j$ is defined as

$$
s_{i j}=\frac{1}{2}\left(c_{i j}+c_{j i}\right)
$$

As an example let us consider the cellular network in Figure 4.12. BS 1 is serving terminal 1 and BS 2 is serving terminal 2. Based on (4.20) we have

$$
c_{12}=\frac{\mathrm{SNR}_{11}}{\mathrm{SINR}_{112}}=\frac{h_{12} p_{2}}{\eta_{0} W}+1 \quad, \quad c_{21}=\frac{\mathrm{SNR}_{22}}{\mathrm{SINR}_{221}}=\frac{h_{21} p_{1}}{\eta_{0} W}+1
$$

Depending on the values of the channel gains $h_{12}$ and $h_{21}$, the two indices can be very different. Hence the similarity index between BS 1 and BS 2 is defined as:

$$
s_{12}=s_{21}=\frac{1}{2}\left(c_{12}+c_{21}\right)=\frac{h_{12} p_{2}+h_{21} p_{1}}{2 \eta_{0} W}+1
$$

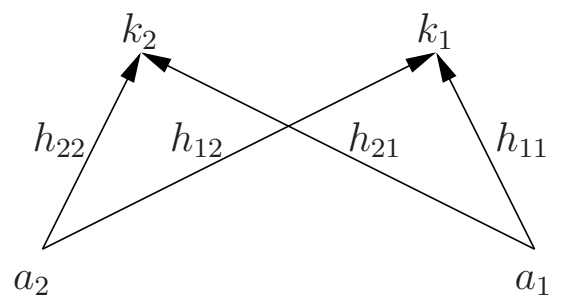

Figure 4.12: A cellular network with two BSs and two terminals

The network similarity matrix $\mathbf{S}=\left[s_{i j}\right] \in \mathbb{R}^{A \times A}$ is formed by the similarity indices of all pairs of BSs in the network. This matrix quantifies the level of interference between any pair of BSs in the network. In order to form this matrix, the channel gains between the terminals and the BSs in the network are required. This information is readily available from the BS assignment stage, where the channel gains of each terminal to the BSs in its vicinity is compared and the terminal is assigned to the BS with the strongest channel gain. The estimated channel gains are reported from each terminal to the corresponding BSs. The reported channel gains are then communicated from the BSs to a central control unit where the similarity matrix is formed. In Section 4.2.3, this 
matrix is employed to develop the network clustering algorithm.

\subsubsection{Clustering}

The key concept in parallel computing is the efficient distribution of the computation load across multiple processors. Firstly, the computations should be assigned to the processors in such a way that there exist little or no inter-processor communication. Secondly, the computation load should be balanced across the processors. A balanced distribution of the computation load does not necessarily mean the assignment of an equal number of computations to all processors as this might not satisfy the first goal of minimizing the inter-processor communication. Thus, the load distribution can be stated as the problem of finding the computation load assignment which minimizes the inter-processor communication, while keeping the computation loads assigned to the processors as equal as possible.

The network clustering problem is analogous to the load distribution problem in parallel computing. Scheduling cells take the place of processors and inter-cell interference takes the place of inter-processor communication. Thus, instead of minimizing the interprocessor communication, the interference levels between the scheduling cells are to be minimized. A balanced distribution of the computation load is replaced by a balanced distribution of the resource allocation load across the scheduling cells, which, in other words, equalizes the resource allocation complexity across the scheduling cells.

The load distribution problem in parallel computing has been well-studied in the context of graph partitioning. There are several ways of posing clustering as a mathematical problem. In order to minimize the inter-dependencies between the clusters the min-cut technique has been proposed:

Let $\mathcal{G}=(\mathcal{V}, \mathcal{E})$ be a fully connected weighted graph with $N$ nodes and a weight matrix 
of $\mathbf{W} \in \mathbb{R}^{N \times N}$. The connectivity between two sets $\mathcal{E}, \mathcal{F} \subset \mathcal{V}$ is defined as

$$
c(\mathcal{E}, \mathcal{F})=\sum_{e \in \mathcal{E}, f \in \mathcal{F}} w_{e f}
$$

where $w_{\text {ef }}$ is the element on row $e$ and column $f$ of the weight matrix $\mathbf{W}$. The min-cut approach generates a $Q$-partition of the graph $\left\{\mathcal{V}_{1}, \cdots, \mathcal{V}_{Q}\right\}$ by minimizing the connectivity of each cluster $\mathcal{V}_{q}$ from the rest of the network $\overline{\mathcal{V}}_{q}$. Mathematically this problem is expressed as

$$
\min _{\left\{\mathcal{V}_{1}, \cdots, \mathcal{V}_{Q}\right\}} \sum_{q=1}^{Q} c\left(\mathcal{V}_{q}, \overline{\mathcal{V}}_{q}\right)
$$

The min-cut solutions often tend to separate individual vertices from the graph which disturbs the desirable balance in the cluster sizes. In order to avoid the generation of too small clusters, the problem is modified as

$$
\min _{\left\{\mathcal{V}_{1}, \cdots, \mathcal{V}_{Q}\right\}} \sum_{q=1}^{Q} \frac{c\left(\mathcal{V}_{q}, \overline{\mathcal{V}}_{q}\right)}{\left|\mathcal{V}_{q}\right|}
$$

By normalizing the connectivity term $c\left(\mathcal{V}_{q}, \overline{\mathcal{V}}_{q}\right)$ by the size of the corresponding set $\left|\mathcal{V}_{q}\right|$, we are essentially enforcing the formation of reasonably large clusters.

The solution of problem (4.26) has been shown to be NP hard. In what follows, we elaborate on how the problem can be relaxed into an easy-to-solve standard linear algebra problem. This technique is known as the spectral clustering technique [34].

\section{Spectral clustering}

Let $\mathbf{U}=\left[\mathbf{u}_{1}, \cdots, \mathbf{u}_{Q}\right] \in \mathbb{R}^{N \times Q}$ be the indicator matrix such that

$$
u_{i j}= \begin{cases}\frac{1}{\sqrt{\left|\mathcal{V}_{j}\right|}} & , v_{i} \in \mathcal{V}_{j} \\ 0 & , v_{i} \notin \mathcal{V}_{j}\end{cases}
$$


and let us define $\mathbf{L}$ as the Laplacian of the weight matrix $\mathbf{W}$ as follows:

$$
\mathbf{L}=\mathbf{D}-\mathbf{W}
$$

where $\mathbf{D}=\left[d_{i j}\right] \in \mathbb{R}^{N \times N}$ is a diagonal matrix with $d_{i i}=\sum_{j=1}^{N} w_{i j}$. It is easy to show that

$$
\mathbf{u}_{q}^{T} \mathbf{L} \mathbf{u}_{q}=\frac{c\left(\mathcal{V}_{q}, \overline{\mathcal{V}}_{q}\right)}{\left|\mathcal{V}_{q}\right|}
$$

Hence,

$$
\sum_{q=1}^{Q} \frac{c\left(\mathcal{V}_{q}, \overline{\mathcal{V}}_{q}\right)}{\left|\mathcal{V}_{q}\right|}=\sum_{q=1}^{Q} \mathbf{u}_{q}^{T} \mathbf{L} \mathbf{u}_{q}=\operatorname{trace}\left(\mathbf{U}^{T} \mathbf{L} \mathbf{U}\right)
$$

and the partitioning problem can be formally written as

$$
\min _{\left\{\mathcal{V}_{1}, \mathcal{V}_{2}, \cdots, \mathcal{V}_{Q}\right\}} \operatorname{trace}\left(\mathbf{U}^{T} \mathbf{L} \mathbf{U}\right)
$$

where $\mathbf{U}$ is defined in (4.27).

By relaxing the constraint on $\mathbf{U}$, the optimization problem can be written as:

$$
\begin{aligned}
& \min _{\left\{\mathcal{V}_{1}, \mathcal{V}_{2}, \cdots, \mathcal{V}_{Q}\right\}} \operatorname{trace}\left(\mathbf{U}^{T} \mathbf{L} \mathbf{U}\right) \\
& \text { subject to } \mathbf{U} \in \mathbb{R}^{N \times Q}
\end{aligned}
$$

From the Rayleigh-Ritz theorem, it is well-known that the optimum $\mathbf{U}$ is formed by the first $Q$ eigen-vectors ${ }^{3}$ of $\mathbf{L}$ as its columns. Hence, $\mathbf{U}$ can be written as $\left[\mathbf{u}_{1}, \mathbf{u}_{2}, \cdots, \mathbf{u}_{Q}\right]$ where $\mathbf{u}_{1}$ is the eigen-vector corresponding to the smallest eigen-value. For any eigen-

\footnotetext{
${ }^{3}$ Corresponding to the $Q$ smallest eigen-values.
} 
vector $\mathbf{u}_{q}=\left[u_{1 q}, u_{2 q}, \cdots, u_{N q}\right]^{T}$, we have

$$
\mathbf{u}_{q}^{T} \mathbf{L} \mathbf{u}_{q}=\sum_{n=1}^{N} \sum_{m=1}^{N}\left(u_{n q}-u_{m q}\right)^{2} w_{n m}
$$

Without loss of generality let us assume the eigen-vectors are normalized, i.e. $\mathbf{u}_{q}^{T} \mathbf{u}_{q}=1$. Hence,

$$
\lambda_{q}=\sum_{n=1}^{N} \sum_{m=1}^{N}\left(u_{n q}-u_{m q}\right)^{2} w_{n m}
$$

Let us consider two vertices $n$ and $m$ with a high $w_{n m}$. For a small eigen-value $\lambda_{q}$, the term $\left(u_{n q}-u_{m q}\right)^{2}$ should be small. Since $\mathbf{U}$ is constructed from the eigen-vectors of the smallest $Q$ eigen-values of $\mathbf{L}$, the term $\left(u_{n q}-u_{m q}\right)^{2}$ should be small for all $q=1, \cdots, Q$. This in turn translates into two geometrically close points in a $Q$-dimensional vector space. Following this argument, each vertex of the graph is represented by a point in the $Q$-dimensional vector space with coordinates $\mathbf{g}_{n} \in \mathbb{R}^{Q}$. In order to form the partition $\left(\mathcal{V}_{1}, \mathcal{V}_{2}, \cdots, \mathcal{V}_{Q}\right)$, the points $\left\{\mathbf{g}_{1}, \mathbf{g}_{2}, \cdots, \mathbf{g}_{N}\right\}$ in the $Q$-dimensional vector space are partitioned based on the K-clustering technique. The pseudo-code for the spectral clustering is provided in Algorithm 3. The choice of K-clustering for the last stage of the spectral clustering method is purely due to the simplicity of this technique as will be discussed in the following.

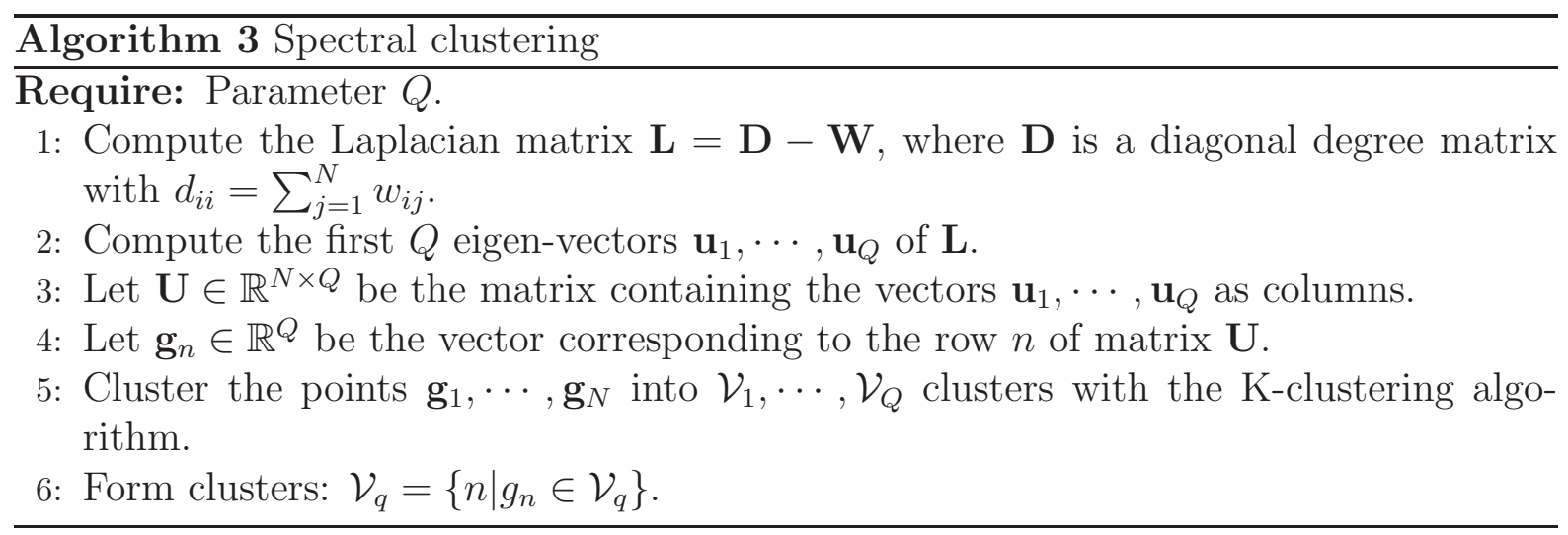

Let us consider a graph of $N$ vertices with the coordinate set $\left\{\mathbf{g}_{1}, \mathbf{g}_{2}, \cdots, \mathbf{g}_{N}\right\}$. Given 
a Q-partition, each cluster $\mathcal{V}_{q}$ can be represented by a cluster-head $\mathbf{m}_{q}$ defined as

$$
\mathbf{m}_{q}=\frac{1}{\left|\mathcal{V}_{q}\right|} \sum_{v \in \mathcal{V}_{q}} \mathbf{g}_{v}
$$

where $\left|\mathcal{V}_{q}\right|$ is the cardinality of $\mathcal{V}_{q}$. It should be noted that the cluster-head does not necessarily have to be one of the vertices and can be any point in the vector space. Given a set of cluster-heads $\left\{\mathbf{m}_{1}, \mathbf{m}_{2}, \cdots, \mathbf{m}_{Q}\right\}$, the corresponding Q-partition can be formed by assigning each vertex of the graph to the cluster-head with the smallest Euclidean distance.

The K-clustering problem is defined as finding the set of $Q$ cluster-heads which minimizes the squared distance from any vertex to its cluster-head. K-clustering is an iterative technique to solve this problem: At the initialization stage, the vertices are randomly clustered into $Q$ clusters $\left\{\mathcal{V}_{1}^{(0)}, \mathcal{V}_{2}^{(0)}, \cdots, \mathcal{V}_{Q}^{(0)}\right\}$. The corresponding cluster-head set $\left\{\mathbf{m}_{1}^{(0)}, \mathbf{m}_{2}^{(0)}, \cdots, \mathbf{m}_{Q}^{(0)}\right\}$ is formed according to (4.35). Given the set of cluster-heads, vertex $v$ with coordinates $\mathbf{g}_{v}$ is reassigned to cluster $q^{*}$ such that

$$
q^{*}=\arg \min _{1 \leq q \leq Q}\left\|\mathbf{g}_{v}-\mathbf{m}_{q}\right\|^{2}
$$

Hence, a new set of $Q$ clusters $\left\{\mathcal{V}_{1}^{(1)}, \mathcal{V}_{2}^{(1)}, \cdots, \mathcal{V}_{Q}^{(1)}\right\}$ is formed. In the subsequent iterations the set of cluster-heads is updated and the vertices are correspondingly reassigned. The procedure is repeated for $L$ iterations. The pseudo-code of this technique is provided in Algorithm 4. It should be noted that:

- the solution of the K-clustering algorithm is a partition of the graph with not exactly but at most $Q$ clusters. In some instances, one or more cluster-heads may not have any vertex associated to them. In this case, a smaller number of clusters are formed. 
- the K-clustering technique is very sensitive to the initialization step. In other words, with a different initial random partition, the technique may result in a very different solution.

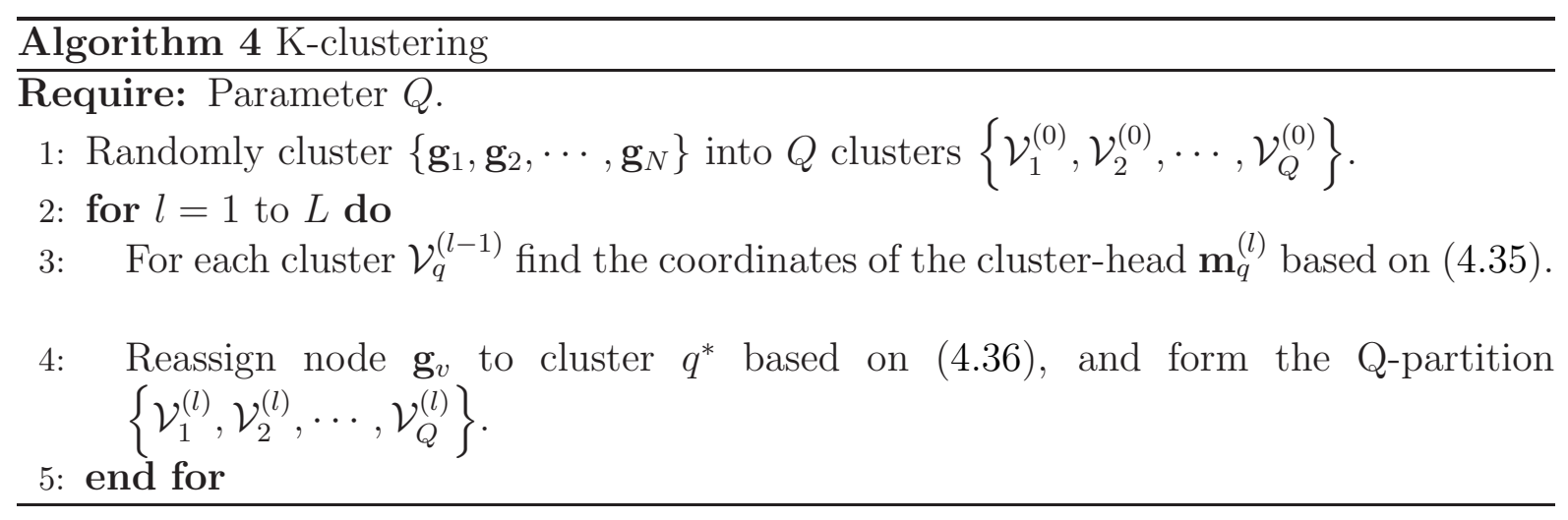

\section{Network clustering algorithm}

In the network graph, each vertex represents a BS. The vertices are all connected and weighted according to the similarity matrix introduced in Section 4.2.2. Thus a fully connected and weighted graph is constructed. The network clustering algorithm forms the scheduling cells. Each scheduling cell is formed by the set of BSs which potentially induce high interference levels on one another. In the network graph, this set of BSs corresponds to the set of vertices which are connected with large weights. Naturally as the potential interference levels between two BSs increase, the coordination between the two BSs becomes more crucial. The BSs forming a scheduling cell will fully coordinate their resource allocation as will be discussed in Section 4.2.4. The proposed resource allocation framework is presented in Section 4.3.

In traditional cellular networks, pre-determined static patterns are employed to decrease the level of interference between the frequency re-use clusters. In the proposed network clustering approach, the key idea is essentially the same: Instead of the static frequency re-use clusters, it is the role of the network clustering algorithm to adaptively form the scheduling cells such that the interference between the clusters are reduced to 
acceptable levels. Essentially, by developing the network clustering algorithm, a new level of adaptation is introduced into the resource allocation framework of cellular networks.

The network clustering algorithm forms a partition of the graph which translates into the formation of the scheduling cells. It should be noted that the scheduling cells are not completely isolated from each other. In other words, interference between the scheduling cells still exits. Nevertheless, as will be seen from the performance evaluation results, an appropriate level of coordination between the BSs, reduces the level of interference between the scheduling cells to acceptable levels such that independent coordination in the scheduling cells becomes possible.

The proposed network clustering algorithm is based on the spectral clustering technique. By applying the spectral clustering method on the network graph, a partition $\mathcal{P}=\left\{\mathcal{V}_{1}, \mathcal{V}_{2}, \cdots, \mathcal{V}_{R}\right\}$ is formed with $R \leq Q$. Due to the intrinsic sensitivity of the Kclustering technique to initialization, the spectral clustering will be performed for $L$ independent initial partitions. Thus $L$ independent partitions of the network $\left\{\mathcal{P}^{(1)}, \mathcal{P}^{(2)}, \cdots, \mathcal{P}^{(L)}\right\}$ are formed. An association matrix $\mathbf{D}=\left[d_{i j}\right] \in[0,1]^{A \times A}$ is defined based on the $L$ partitions as follows:

$$
d_{i j}=\frac{\text { Number of times BS } i \text { and BS } j \text { reside in the same cluster }}{L}
$$

The BSs $i$ and $j$ with an association factor $d_{i j}$ of larger than a threshold $T$ become part of the same scheduling cell. The network clustering strategy is provided in Algorithm 5.

Parameter $Q$ defines the maximum number of clusters in a K-clustering problem. In the proposed algorithm, $Q$ limits the maximum number of clusters in each iteration $l$. A higher $Q$ results in a lower number of BSs per scheduling cell which translates into less coordination in resource allocation. The effect of $Q$ on the cluster size is studied in Section 4.4. A larger $T$ in Algorithm 5 requires two BSs to have a larger association factor to join the same scheduling cell. Hence the value of $T$ directly impacts the size of the scheduling cells. With a $T$ of unity, no two BSs will join the same scheduling cell as 


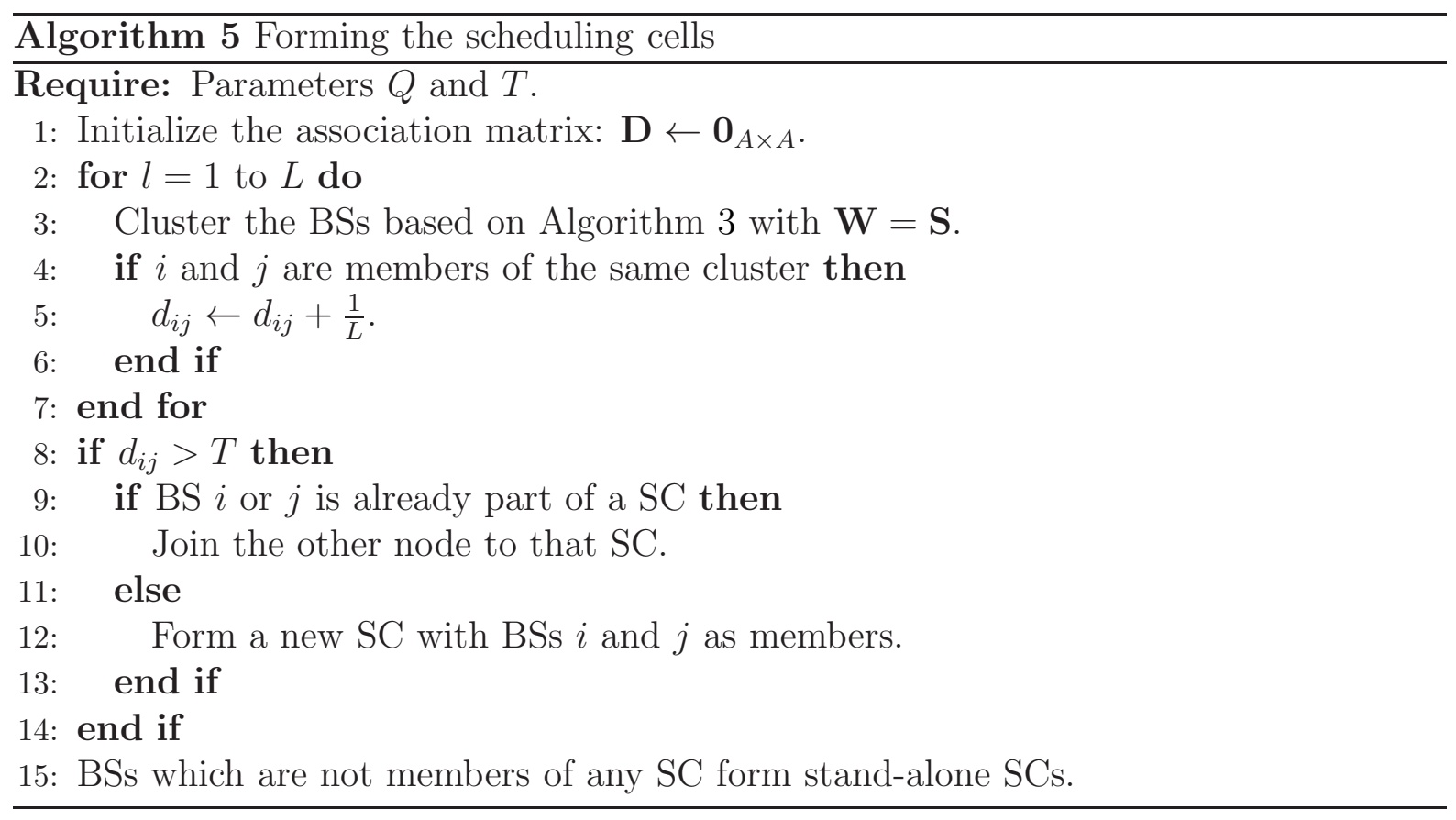

$d_{i j}<1$ for any $i \neq j$. On the other hand, a $T$ of zero translates into full coordination among all BSs. This is due to the fact that $d_{i j}>0$ for any $i \neq j$. The resource allocation framework is discussed in section 4.3. In the proposed framework, a fixed value for $T$ has been considered. The size of the scheduling cells are adjusted by varying the system parameter $Q$.

\subsubsection{Coordinated resource allocation}

In time slot $t$ the data rate of terminal $l$ on frequency channel $n$ is

$$
r_{l n}(t)=c_{l n}(t) \log _{2}\left(1+\frac{\mathrm{SINR}_{l n}(t)}{2}\right)
$$

where $c_{l n}(t)$ is equal to zero if frequency channel $n$ is not assigned to terminal $l$ in time slot $t$ and is proportional to the number of sub-carriers in frequency channel $n$ otherwise. $\operatorname{SINR}_{l n}(t)$ is the SINR level of the link of terminal $l$ on frequency channel $n$ in time slot $t$.

In a multi-carrier system with $N$ frequency channels, the data rate of terminal $l$ in 
time slot $t$ is the sum of the data rates of the terminal on the $N$ frequency channels and is denoted by $r_{l}(t)$.

$$
r_{l}(t)=\sum_{n=1}^{N} r_{l n}(t)
$$

After time slot $t$, the average rate of terminal $l$ is updated according to

$$
\bar{r}_{l}(t)=\frac{(t-1) \bar{r}_{l}(t-1)+r_{l}(t)}{t}=\left(1-\frac{1}{t}\right) \bar{r}_{l}(t-1)+\frac{1}{t} r_{l}(t)
$$

where the average rate at the beginning of the first time slot is set to zero for all terminals, i.e. $\bar{r}_{l}(0)=0$.

The resource allocation in each scheduling cell is coordinated. The resource allocation problem at the beginning of time slot $t$ is formulated as

$$
\max \sum_{k_{l} \in \mathcal{K}(c)} \log \bar{r}_{l}(t)
$$

where $\mathcal{K}(c)$ is the set of terminals in scheduling cell $c$. For the ease of notation, the scheduling cell index $c$ is dropped in the rest of this section.

The summand of the resource allocation problem can be simplified as

$$
\begin{aligned}
\log \bar{r}_{l}(t) & =\log \left[\left(1-\frac{1}{t}\right) \bar{r}_{l}(t-1)\left(1+\frac{1}{t-1} \cdot \frac{r_{l}(t)}{\bar{r}_{l}(t-1)}\right)\right] \\
& =\log \left[\left(1-\frac{1}{t}\right) \bar{r}_{l}(t-1)\right]+\log \left(1+\frac{1}{t-1} \cdot \frac{r_{l}(t)}{\bar{r}_{l}(t-1)}\right)
\end{aligned}
$$

For any $x \ll 1, \log (1+x)$ can be approximated by $x$. Assuming $\frac{1}{t-1} \cdot \frac{r_{l}(t)}{\bar{r}_{l}(t-1)} \ll 1$, we have

$$
\log \bar{r}_{l}(t) \approx \log \left[\left(1-\frac{1}{t}\right) \bar{r}_{l}(t-1)\right]+\frac{1}{t-1} \cdot \frac{r_{l}(t)}{\bar{r}_{l}(t-1)}
$$


Since at the beginning of time slot $t$ the first term is known (constant), the optimization problem (4.41) can be approximated by

$$
\max \sum_{k_{l} \in \mathcal{K}} \frac{r_{l}(t)}{\bar{r}_{l}(t-1)}
$$

Based on (4.39), in a multi-carrier system this problem can be written as follows:

$$
\max \sum_{k_{l} \in \mathcal{K}} \sum_{n=1}^{N} \frac{r_{l n}(t)}{\bar{r}_{l}(t-1)}
$$

The scheduling cell is formed by a set of BSs. Each BS has a fixed transmission power budget. The power budget is assumed to be uniformly distributed across the available frequency channels. Hence in a given time slot and on a given frequency channel, a BS either transmits with a fixed transmit power or not transmit at all. With the assumption of equal power distribution across the available frequency channels, there is no longer any inter-dependency between the resource allocation of the frequency channels. In other words, the optimization problem in (4.44) can be solved on a per-frequency channel basis. Thus, for frequency channel $n$ the following optimization problem should be solved:

$$
\max \sum_{k_{l} \in \mathcal{K}} \frac{r_{l n}(t)}{\bar{r}_{l}(t-1)}
$$

In the remainder of this section, a system with one frequency channel is assumed and the frequency channel index $n$ is dropped.

Let us assume that we know which BSs transmit in time slot $t$. In other words, let us fix the BS transmission power levels in time slot $t$. In this case, Section 4.2.4 shows that each BS can independently decide on which terminal to serve in time slot $t$ in the scheduling cell. In Section 4.2.4 we assume that the set of terminals to be served in time slot $t$ is known and discuss how the transmission powers should be accordingly optimized. Subsequently in Section 4.2.4, the joint resource allocation problem is studied. 


\section{User scheduling with fixed transmission power levels}

Let $\mathbf{p}(t)=\left[p_{1}, \cdots, p_{|A|}\right]^{T}$ be the transmission power vector of the BSs in the scheduling cell in time slot $t$. Each terminal receives a desired signal from the serving BS and interfering signals from all or a subset of BSs in the network. With a fixed power vector p, each BS knows the received signal power levels from all BSs in the scheduling cell at each of its assigned terminals. Hence, BS $i$ can estimate ${ }^{4}$ the supported data rate of the terminals it is serving. Accordingly, each BS allocates the frequency channel to terminal $k_{a}^{*}(t)$ based on the following proportional fairness criterion:

$$
k_{a}^{*}(t)=\arg \max _{k_{l} \in \mathcal{K}_{a}} \frac{r_{l}(t)}{\bar{r}_{l}(t-1)} \quad, \forall a \in \mathcal{A}
$$

For a given power vector $\mathbf{p}$, the set of the chosen terminals $\left\{k_{a}^{*}(t)\right\}_{a \in \mathcal{A}}$ forms the cochannel user set $\mathcal{K}(\mathbf{p}, t)$ in time slot $t$.

\section{Power control with a fixed co-channel user set}

Let us assume that the co-channel user set is known for time slot $t$ and let us denote it by $\mathcal{K}(t)$. The resource allocation problem at time slot $t$ can be written as

$$
\mathbf{p}^{*}(t)=\arg \max _{\mathbf{p}} \sum_{k_{l} \in \mathcal{K}(t)} \log \bar{r}_{l}(t)
$$

Based on (4.44), the optimum power vector can be obtained as

$$
\mathbf{p}^{*}(t)=\arg \max _{\mathbf{p}} \sum_{k_{l} \in \mathcal{K}(t)} \frac{r_{l}(t)}{\bar{r}_{l}(t-1)}
$$

This is essentially a power control problem. In [37], it is shown that this weighted sum rate problem is not convex and based on the first-order optimality conditions an iterative

\footnotetext{
${ }^{4}$ It should be noted that due to the lack of knowledge of out-of-cell interference, the actual supported data rate of each terminal is not available. However, an estimate can be obtained based on the in-cell interference which is known due to the coordination within each scheduling cell.
} 
method is proposed as follows:

Let us assume that at BS $i$ the scheduler (4.46) has decided to serve terminal $l$ and BS $j$ has decided to serve terminal $k$. The main idea of the iterative power control algorithm is to update the power levels based on the Karush-Kuhn-Tucker (KKT) conditions which should be satisfied by any local optimum of the optimization problem. Hence, the derivative of the objective function is set to zero.

$$
\frac{\partial}{\partial p_{i}} \sum_{k_{l} \in \mathcal{K}(\mathbf{p}, t)} w_{l}(t) r_{l}(t)=0
$$

or

$$
\frac{w_{l} h_{l i}}{p_{i} h_{l i}+2\left(\eta_{0} W+\sum_{j \neq i} p_{j} h_{l j}\right)}=\sum_{j \neq i} t_{j i}
$$

where

$$
t_{j i}=w_{k} \frac{2 h_{k i}}{p_{j} h_{k j}}\left(\frac{\left(\mathrm{SINR}_{j}\right)^{2}}{1+\mathrm{SINR}_{j}}\right) \quad, \quad \operatorname{SINR}_{j}=\frac{p_{j} h_{k j}}{2\left(\eta_{0} W+\sum_{i \neq j} p_{i} h_{l i}\right)}
$$

Rewriting (4.50), the transmission power of BS $i$ is derived as

$$
p_{i}=\left[\frac{w_{l}}{\sum_{j \neq i} t_{j i}}-\frac{2\left(\eta_{0} W+\sum_{j \neq i} p_{j} h_{l j}\right)}{h_{l i}}\right]_{0}^{P_{\max }}
$$

where the transmission power is lower-bounded by 0 and upper-bounded by $P_{\max }$. The power control algorithm iterates over (4.52) for all BSs until the power levels converge. The convergence of this method has been proved [12], however, the solution is only guaranteed to be a local optimum.

\section{Combined user scheduling and power control}

In [33], the weighted sum rate problem (4.45) has been extensively studied and three iterative algorithms have been proposed. A similar iterative algorithm is later proposed 
in [38].

Let $\mathbf{p}^{(0)}$ denote a given initial power vector. In the first iteration, the co-channel user set $\mathcal{K}\left(\mathbf{p}^{(0)}\right)$ is formed from (4.46). With $\mathcal{K}\left(\mathbf{p}^{(0)}\right)$, the power vector is updated to $\mathbf{p}^{(1)}$ which maximizes (4.48). In the second iteration, given $\mathbf{p}^{(1)}$, the user set $\mathcal{K}\left(\mathbf{p}^{(1)}\right)$ is formed and the power vector $\mathbf{p}^{(2)}$ is similarly derived. The procedure is iterated $L$ times until convergence. The pseudo-code of this technique is provided in Algorithm 6.

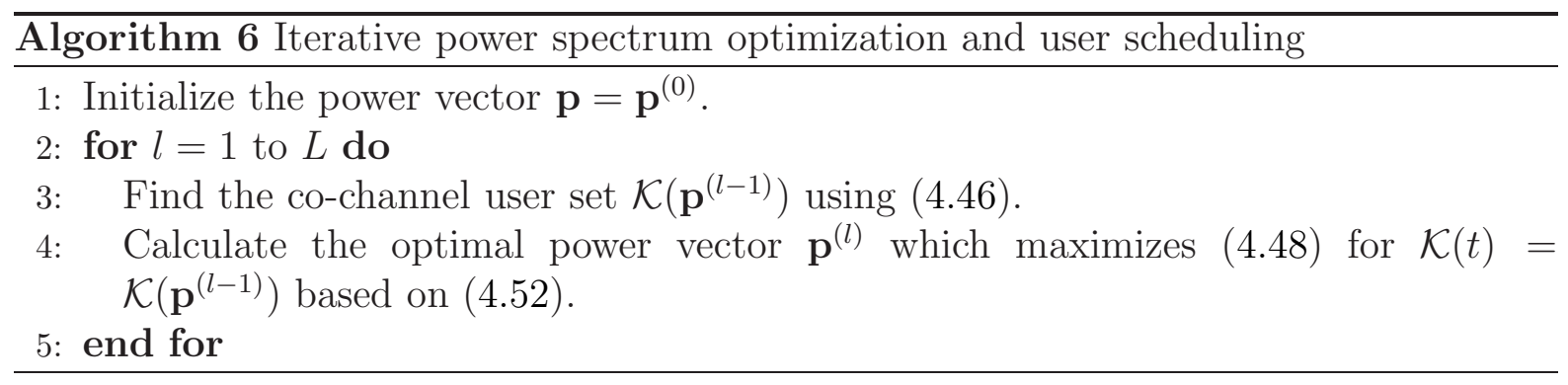

Clearly, the final result of this process is dependent on the choice of the initial power vector $\mathbf{p}^{(0)}$. Hence, an appropriate initialization is required. In [33], this issue has been addressed and an initialization procedure has been proposed. The optimization problem (4.48) is first solved assuming $p_{i} \in\left\{P_{\max }, 0\right\}$. The solution of this binary power control problem (BPC) then serves as the initial point for the iterative Algorithm 6. However, the performance of the BPC is compared with that of the three proposed algorithms and the results show that the iterative method yields little improvement to the performance of the BPC.

Following these arguments, the BPC solution will be considered for the coordinated resource allocation in the scheduling cell. For a scheduling cell with $A$ BSs, there are $2^{A}$ possible transmission power vectors. At time slot $t$, for each power vector $\mathbf{p} \in\left\{0, P_{\max }\right\}^{2^{A} \times 1}$ the corresponding co-channel user set $\mathcal{K}(\mathbf{p}, t)=\left\{k_{a}^{*}(t)\right\}_{a \in \mathcal{A}}$ is formed according to

$$
k_{a}^{*}(t)=\arg \max _{k_{l} \in \mathcal{K}_{a}} \frac{r_{l}(t)}{\bar{r}_{l}(t-1)} \quad, \forall a \in \mathcal{A}
$$


The optimal power vector at time slot $t$ is then the one which satisfies

$$
\mathbf{p}^{*}(t)=\arg \max _{\mathbf{p}} \sum_{k_{l} \in \mathcal{K}(\mathbf{p}, t)} \log \bar{r}_{l}(t)
$$

The complexity of this approach increases exponentially with the number of BSs in a scheduling cell. However, this complexity is tunable by adjusting the average size of the scheduling cells as will be discussed in Section 4.4 .

\subsection{Resource allocation framework}

The proposed resource allocation framework is comprised of two levels of abstraction:

- Level A: In the first level, the scheduling cells are formed according to the network clustering algorithm developed in Section 4.2.3. The network is clustered into scheduling cells according to the deployment pattern of the BSs and the distribution of the active terminals. The size of the scheduling cells can be adjusted based on the system designer's preferences by varying system parameter $Q$. The resource allocation is coordinated among the BSs forming a scheduling cell. With a larger scheduling cell, a higher coordination in resource allocation improves the network performance at the cost of a higher degree of complexity.

- Level B: In the second level, the system resources are allocated to communication links independently in each scheduling cell at each time slot. The resource allocation is based on a coordinated proportional fairness strategy developed in Section 4.2.4.

The flowchart of the framework is provided in Figure 4.13. The coordinated resource allocation at level B is performed once every Transmission Time Interval (TTI), also known as a sub-frame (SF) in the LTE terminology. The network clustering at level A is performed at a larger time scale. The clustering algorithm re-forms the scheduling cells once every $U$ sub-frames. The system parameter $U$ primarily depends on the rate 
of variations in the wireless network and environment. For a fast time-varying network, a small $U$ translates into fast re-formation of the scheduling cells. The parameter $U$ is essentially a design parameter which should be adjusted according to the environment. As an example, let us assume that the shadow fading changes with a displacement in the order of tens of wavelengths. For a system with a center frequency of $2 \mathrm{GHz}$ and a terminal velocity of $30 \mathrm{Kmph}$, a terminal travels ten wavelengths in 0.18 seconds which is equivalent to 18 frames in the LTE system. Consequently, a $U$ of a few tens of subframes will be a reasonable choice. The pseudo-code of the framework is presented in Algorithm 7.

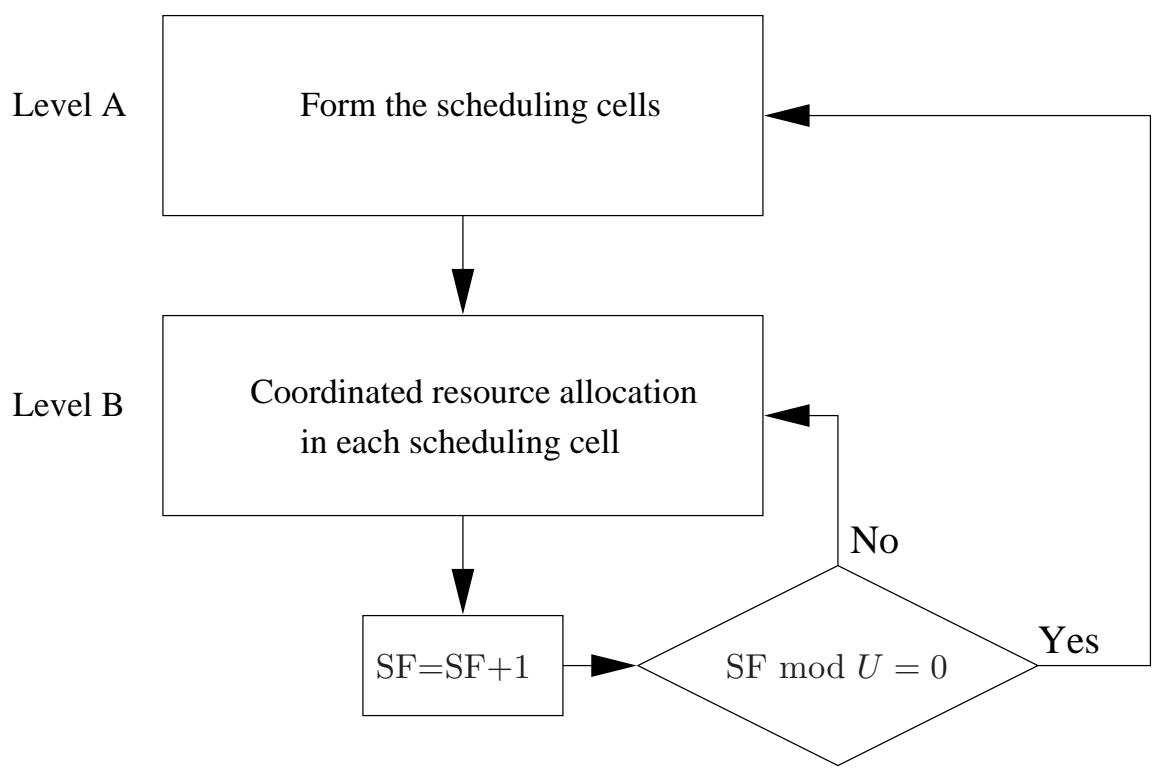

Figure 4.13: Resource allocation framework with two levels of abstraction

\subsection{Numerical results}

Performance evaluation results have been provided for the traditional cellular network in Section 4.4.1. In Section 4.4.2, the autonomous cellular network is introduced and the performance evaluation results are discussed. 


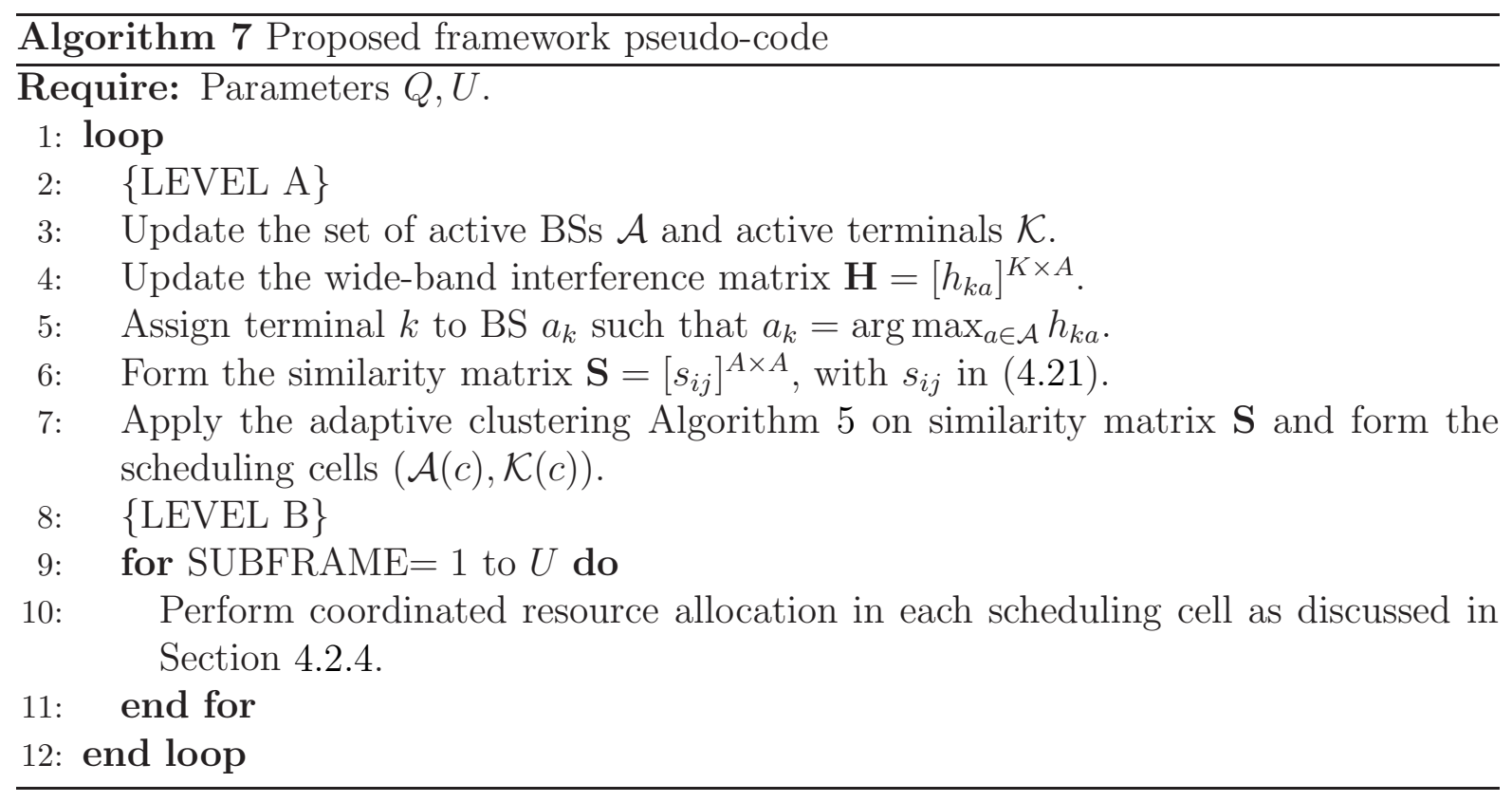

\subsubsection{Traditional cellular network}

A cellular network composed of $21 \mathrm{BSs}$ with regular deployment according to the hexagonal pattern is considered. Each BS is located at the center of the cell with an omnidirectional antenna. An inter-site-distance of 500 meters is assumed. The channel gains between the terminals and BSs are modeled based on the ITU recommendations in an urban-macro environment [2]. The simulation parameters are provided in Table 4.1. In order to comply with the LTE air interface technology the system bandwidth has been chosen to be equal to that of a sub-carrier in the standard. In addition, each time slot has been chosen to be equal to a sub-frame size of $1 \mathrm{~ms}$. In each time slot 14 transmissions (in time) occur for the scheduled terminal which corresponds to the 14 OFDM symbols in a resource block. In addition, it should be noted that the performance evaluation is conducted assuming a fixed value for the threshold parameter $T$. The size of the scheduling cells is controlled with varying the K-clustering parameter $Q$.

The system performance of the proposed technique is compared with the following two traditional techniques:

- Universal frequency reuse (UFR): In each cell and at each time slot, the scheduling 
Table 4.1: System parameters

\begin{tabular}{|c|c|}
\hline System parameter & Value \\
\hline \hline Carrier frequency & $2 \mathrm{GHz}$ \\
\hline Bandwidth $(W)$ & $15 \mathrm{KHz}$ \\
\hline Sub-frame size (time slot duration) & $1 \mathrm{~ms}$ \\
\hline Number of simulated sub-frames $\left(t_{s}\right)$ & 40 \\
\hline BS power budget $\left(P_{\max }\right)$ & $18.22 \mathrm{dBm}$ \\
\hline Noise figure at terminal & $7 \mathrm{~dB}$ \\
\hline Background noise power spectral density & $-174 \mathrm{dBm} / \mathrm{Hz}$ \\
\hline Terminal velocity & $30 \mathrm{Kmph}$ \\
\hline Number of BSs $(M)$ & 21 \\
\hline Total number of terminals & $4,6,8,10$ per BS \\
\hline Traffic model & Full buffer \\
\hline Clustering algorithm threshold $(T)$ & 0.7 \\
\hline
\end{tabular}

is based on the proportional fairness criterion. No reuse is allowed within each cell.

- Static cluster size of 3: In our terminology, a cluster size of 3 would translate into regular scheduling cells with 3 BSs per scheduling cell. In order to study the effect of adaptive clustering (vs. static clustering), the scheduling in this network is also based on the coordinated scheduling approach proposed in Section 4.2.4.

The proposed clustering algorithm clusters the BSs in the network adaptively. The average size of the cluster is a function of the clustering parameter $Q$. In Figure 4.14 the average size of the clusters is depicted for different values of this parameter. The curve has been plotted for different number of terminals which, as illustrated in this figure, approximately yield the same result. A higher $\mathrm{Q}$ results in more clusters which consequently decreases the average cluster size in the network. Essentially $Q$ is a system parameter, equivalent to the frequency reuse factor in the traditional networks, which adjusts the level of BS coordination and hence the complexity in resource allocation. The performance of the proposed technique is evaluated for two values of $Q=4$ and $Q=8$ which correspond to average cluster sizes of 3.3 and 1.7 respectively. 
Let $\bar{\eta}_{l}$ be the average spectral efficiency of terminal $l$ defined as

$$
\bar{\eta}_{l}=\frac{\bar{r}_{l}\left(t_{s}\right)}{W}
$$

where the terminal average rates are calculated over $t_{s}=40$ sub-frames. The cell spectral efficiency of the network is defined as the sum spectral efficiency of all terminals in the network normalized by the number of BSs $M$.

$$
\bar{\eta}=\frac{\sum_{l=1}^{K} \bar{\eta}_{l}}{M}
$$

With $\mathbf{s}=\left[\bar{\eta}_{1}, \cdots, \bar{\eta}_{K}\right]$ denoting the vector of average spectral efficiencies of all terminals in the network. The $5 \%$ point of the cumulative distribution function (CDF) of the elements of $\mathbf{s}$ is defined as the cell edge spectral efficiency of the network.

The cell spectral efficiency performance is illustrated in Figure 4.15. With $Q=4$ which translates into an average cluster size of $3.3 \mathrm{BSs}$, the cell spectral efficiency is on average $10.7 \%$ higher than that of the UFR technique. Compared to a static cluster size of 3 , the spectral efficiency has improved by $2.4 \%$. Increasing $Q$ to 8 translates into an average cluster size of 1.7 . The spectral efficiency improves by $5.5 \%$ when compared with UFR. It achieves $97.7 \%$ of the cell spectral efficiency of the static clustering technique. In other words, by reducing the cluster size (or equivalently the resource allocation complexity) from 3 to 1.7 , i.e. $43.4 \%$, the loss in performance is merely $2.3 \%$.

The cell edge spectral efficiency performance is illustrated in Figure 4.16. With $Q=4$ which translates into an average cluster size of 3.3 BSs, the cell edge spectral efficiency is on average $53.1 \%$ higher than that of the UFR technique. Compared to a static cluster size of 3 , the cell edge spectral efficiency has improved by $5.5 \%$. Increasing $Q$ to 8 translates into an average cluster size of 1.7. The cell edge spectral efficiency improves by $24 \%$ when compared with UFR. It achieves $85.5 \%$ of the cell spectral efficiency of the static clustering technique. In other words, by reducing the cluster size (or equivalently 
the resource allocation complexity) from 3 to 1.7 , i.e. $43.4 \%$, the loss in performance is merely $14.5 \%$.

The simulation results show that the coordinated resource allocation scheme yields a better performance than the universal frequency reuse strategy. The level of improvement depends on the level of coordination between the BSs which can be tuned by adjusting parameter $Q$. It is important to note that static clustering is only possible when the BSs are identical and regularly deployed. In an autonomous cellular network, the BSs can have different power budgets and are deployed in an irregular fashion. In this case a static clustering of the network is not possible. The proposed framework, on the other hand, can adaptively form the scheduling cells based on the time varying network topology. The performance of the proposed framework in an autonomous network is studied in section 4.4.2.

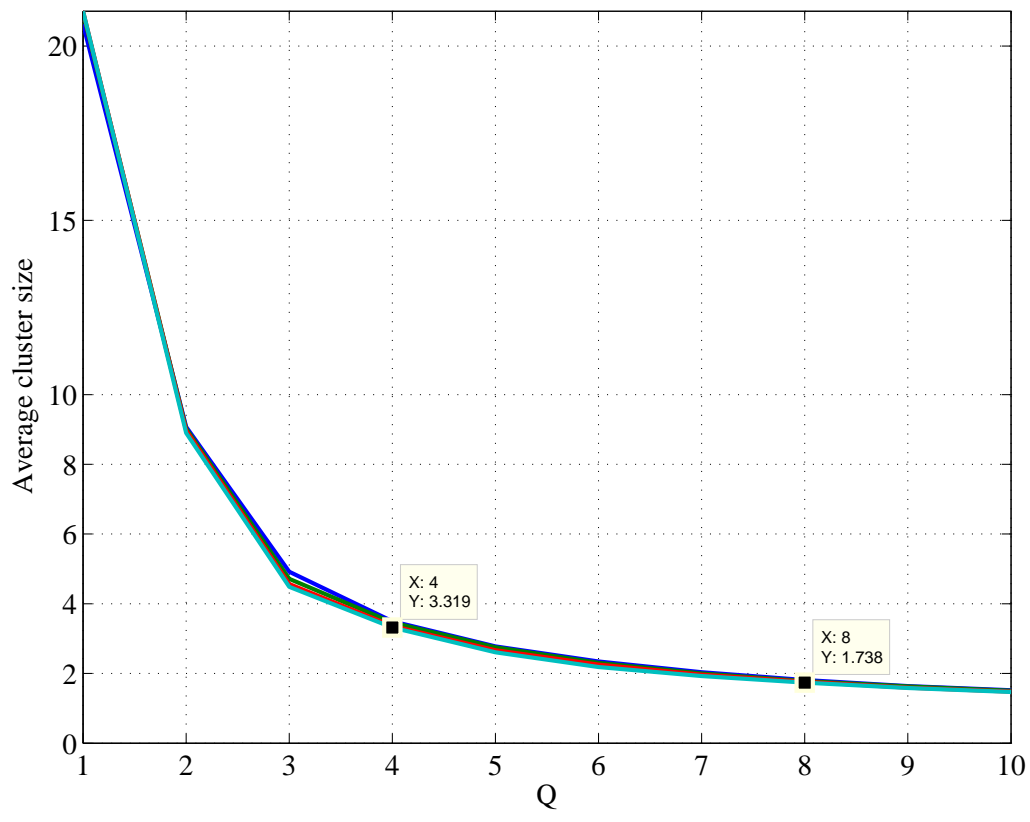

Figure 4.14: Average cluster size vs. the K-clustering parameter $Q$ 


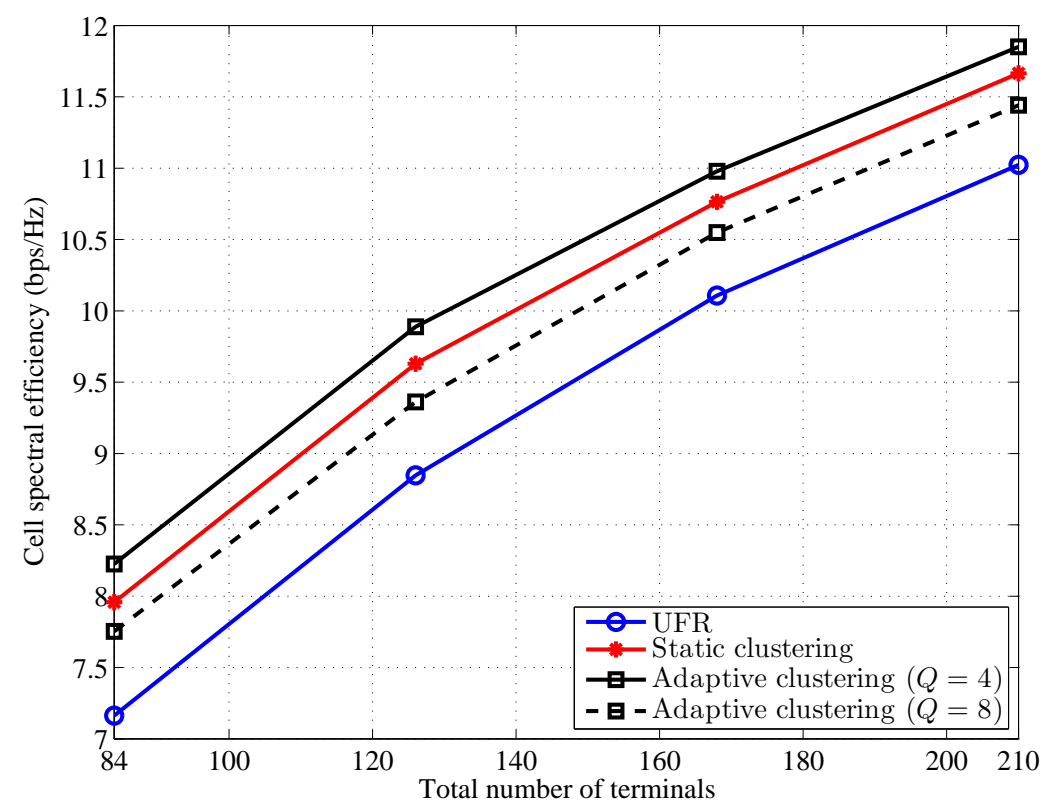

Figure 4.15: Cell spectral efficiency vs. the total number of terminals in the network

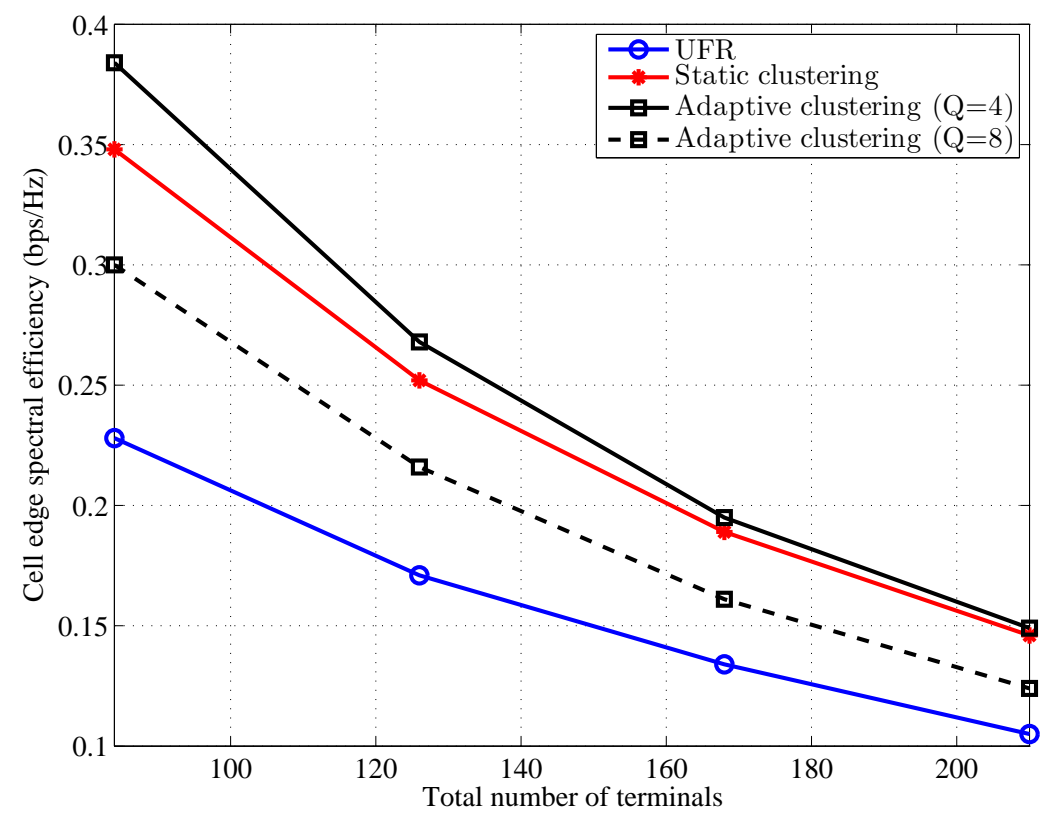

Figure 4.16: Cell edge spectral efficiency vs. the total number of terminals in the network 


\subsubsection{Autonomous cellular network}

The BSs serve a set of terminals in the network coverage area. Due to the irregularity of the network infrastructure, most of the classical resource allocation techniques for cellular networks cannot be employed. The traditional frequency reuse clusters were developed based on the assumption of the regularity of the BS deployments. The more recently proposed fractional frequency reuse techniques are also developed assuming a regular deployment of the BSs. In fact, the Universal Frequency Reuse (UFR) is the only traditional method which can be considered. In this strategy, the system frequency resources are available to all BSs. With no coordination between the BSs, all BSs in the network transmit simultaneously on all time slots and all frequency channels. This can result in excessive interference levels and consequently a poor network performance.

Figure 4.17 illustrates the coverage area of a traditional cellular network with 19 BSs, where $R$ denotes the cell radius. In the autonomous cellular network the same number of BSs are deployed in the same coverage area. The network coverage area is modeled as the circle of radius $4.5 R$. A random two-dimensional uniform distribution is considered for the deployment of the BSs across the network coverage area. Similarly, the terminals have a uniform distribution across the network coverage area.

The system parameters are provided in Table 4.2. The model developed in Section 4.1 is utilized for the generation of the shadow fading gains. In order to decrease the simulation load, the performance is evaluated for a system with a single frequency channel. The system bandwidth has been chosen to be equal to that of a sub-carrier in the LTE standard. In addition, each time slot has been chosen to be equal to a sub-frame of 1ms. In each time slot 14 transmissions (in time) occur for the scheduled terminal which corresponds to the 14 OFDM symbols in a resource block.

The size of the scheduling cells depends on the density of the BS deployments. While a cluster of densely deployed BSs forms one large scheduling cell, a sparse deployment of BSs will form a number of smaller scheduling cells. Albeit in the proposed framework, 


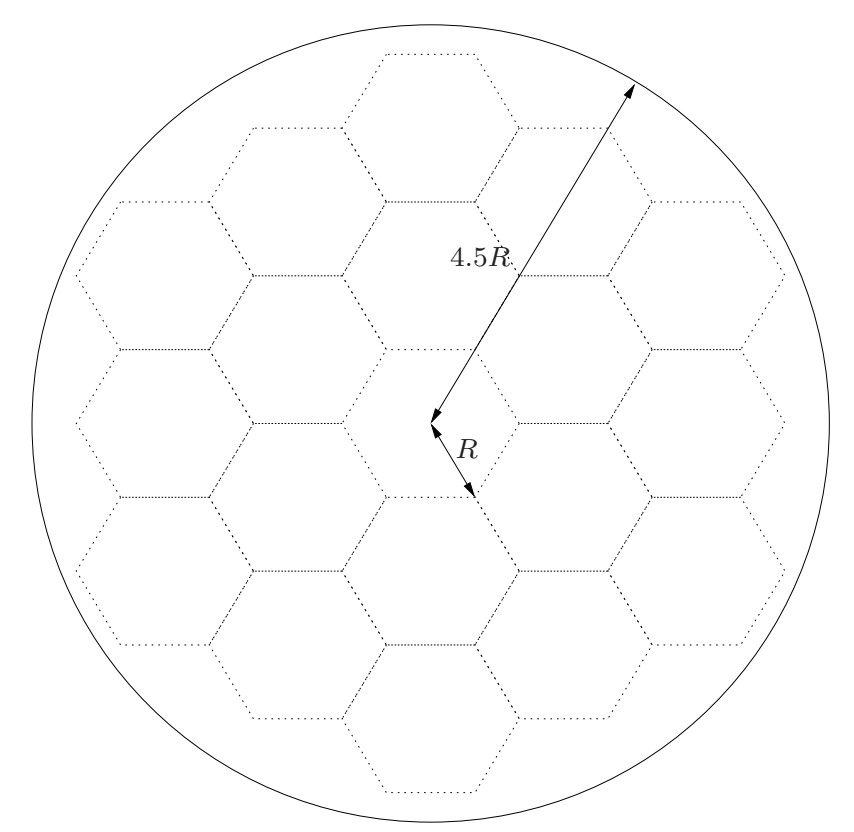

Figure 4.17: Network coverage area for a cellular network with 19 BSs

Table 4.2: System parameters

\begin{tabular}{|c|c|}
\hline System parameter & Value \\
\hline \hline Carrier frequency & $2 \mathrm{GHz}$ \\
\hline Bandwidth $(W)$ & $15 \mathrm{KHz}$ \\
\hline Sub-frame size (time slot duration) & $1 \mathrm{~ms}$ \\
\hline Number of simulated sub-frames $\left(t_{s}\right)$ & 40 \\
\hline BS power budget $\left(P_{\max }\right)$ & $18.22 \mathrm{dBm}$ \\
\hline Noise figure at terminal & $7 \mathrm{~dB}$ \\
\hline Background noise power spectral density & $-174 \mathrm{dBm} / \mathrm{Hz}$ \\
\hline Terminal velocity & $30 \mathrm{Kmph}$ \\
\hline Number of BSs $(M)$ & 19 \\
\hline Total number of terminals & $4,6,8,10$ per BS \\
\hline Traffic model & Full buffer \\
\hline
\end{tabular}


the average size of the scheduling cell can be adjusted by the clustering parameter $Q$. The average size of the scheduling cells reflects the level of coordination between the BSs. In Figure 4.18, the average size of the scheduling cell is plotted as a function of the clustering parameter $Q$. This figure shows that a smaller $Q$ would increase the average size of the scheduling cells which in turn translates into coordination between a larger number of BSs. For instance, a $Q$ of 8 results in an average scheduling cell size of 1.7 BSs. In other words, on average the resource allocation between every $1.7 \mathrm{BSs}$ is coordinated. With a $Q$ of 4 the level of coordination increases to between an average of 3.5 BSs. It is important to note that the position and the number of the active terminals also play a key role in the network clustering. In Figure 4.19, the average size of the scheduling cell is plotted as a function of the total number of active terminals. This figure illustrates that the average scheduling cell size is approximately constant with different number of terminals. In the UFR method, there is no coordination between the BSs which is equivalent to a constant scheduling cell size of unity.

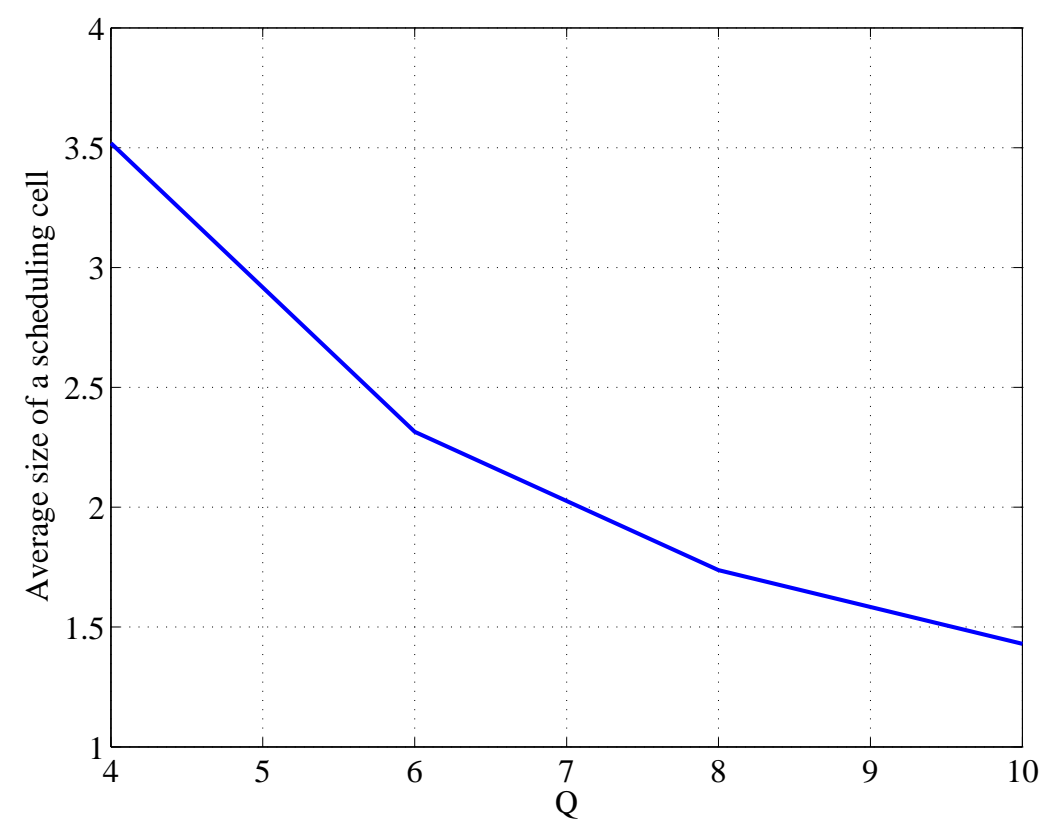

Figure 4.18: Average size of the scheduling cells vs. the clustering parameter $Q$

In this part we study the effect of $Q$ on the network performance. The performance 


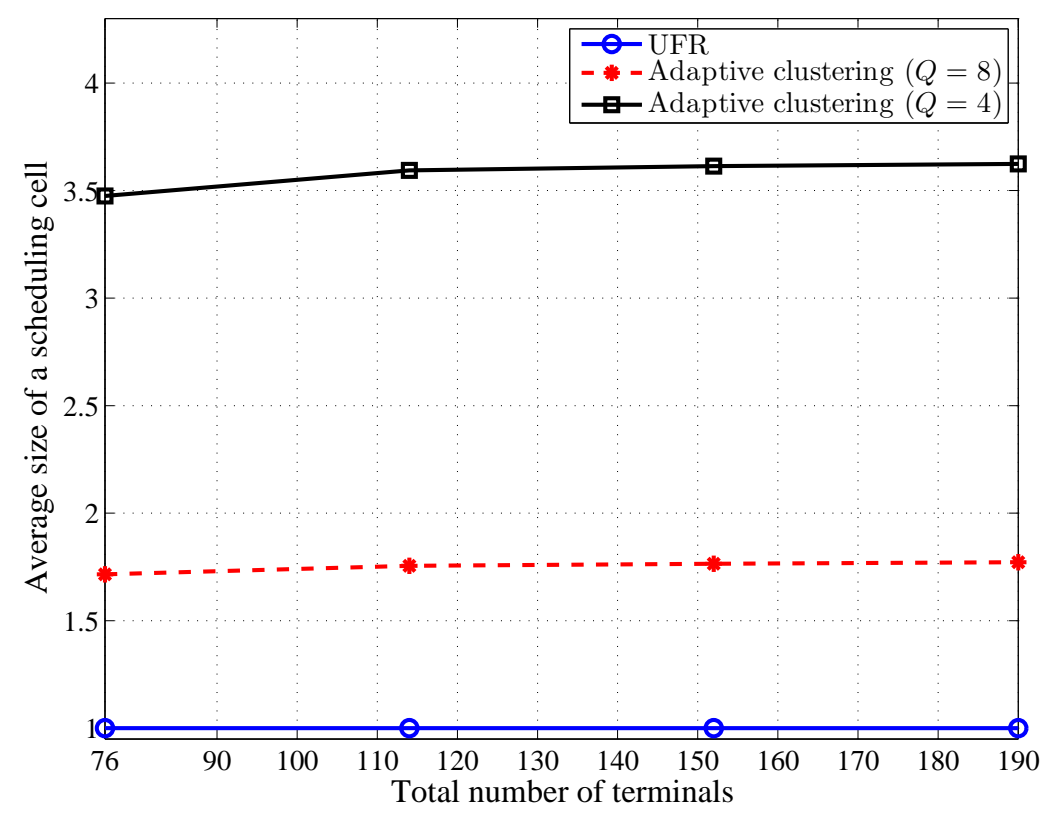

Figure 4.19: Average size of the scheduling cells vs. the total number of terminals in the network

is evaluated for a total number of 76, 114 and 152 active terminals (which respectively correspond to an average of 4,5 and 6 terminals per BS). The cell spectral efficiency is plotted as a function of $Q$ in Figure 4.20. Based on Figure 4.18, a $Q$ of 10 translates into an average cluster size of 1.4 and a $Q$ of 4 results in an average cluster size of 3.5. Hence, decreasing $Q$ from 10 to 4 is equivalent to increasing the coordination level in the network by a factor of 2.5. For a network with 152 active terminals, this increase in the coordination level results in a $6.4 \%$ improvement in the cell spectral efficiency. The cell edge spectral efficiency improves by $35.5 \%$. The cell edge spectral efficiency results are provided in Figure 4.21. In what follows, the network performance of the proposed framework is evaluated for the cases of $Q=4,8$.

The cell spectral efficiency of the proposed framework is plotted as a function of the total number of terminals in Figure 4.22. The cell spectral efficiency of the UFR strategy in the autonomous cellular network (denoted by 'UFR') together with the cell spectral efficiency of the traditional cellular network (denoted by 'Traditional network (UFR)') 


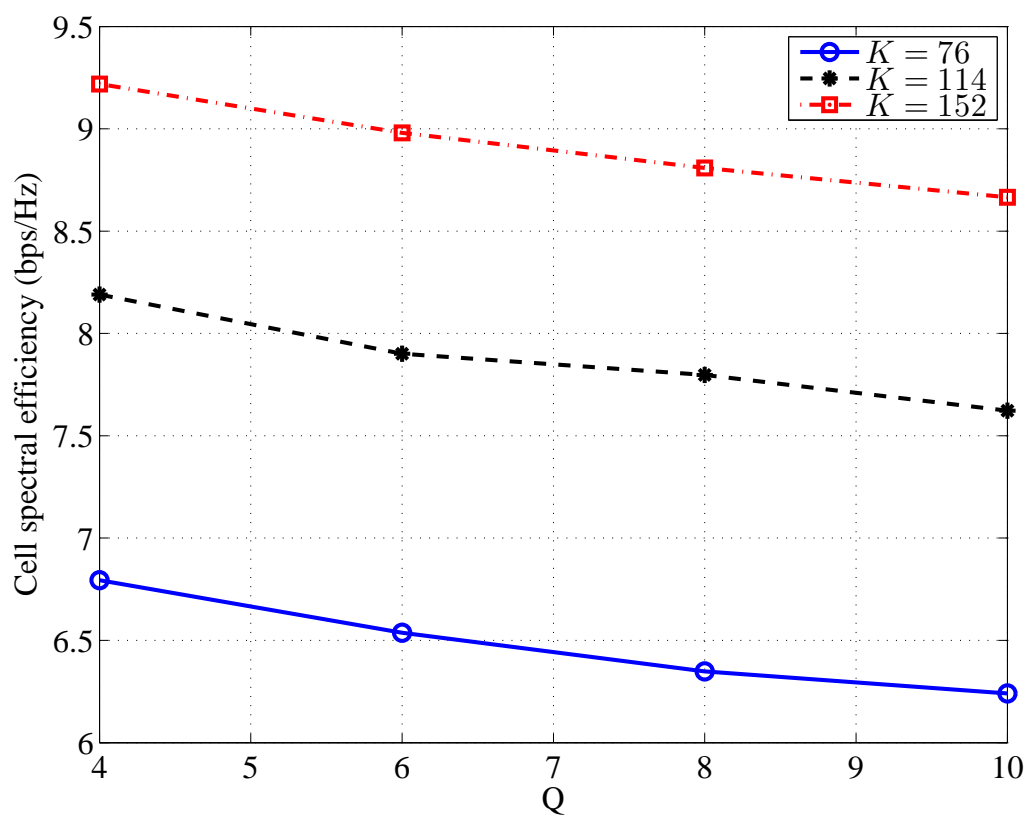

Figure 4.20: Cell spectral efficiency vs. the clustering parameter $Q$ for different total number of terminals in the network

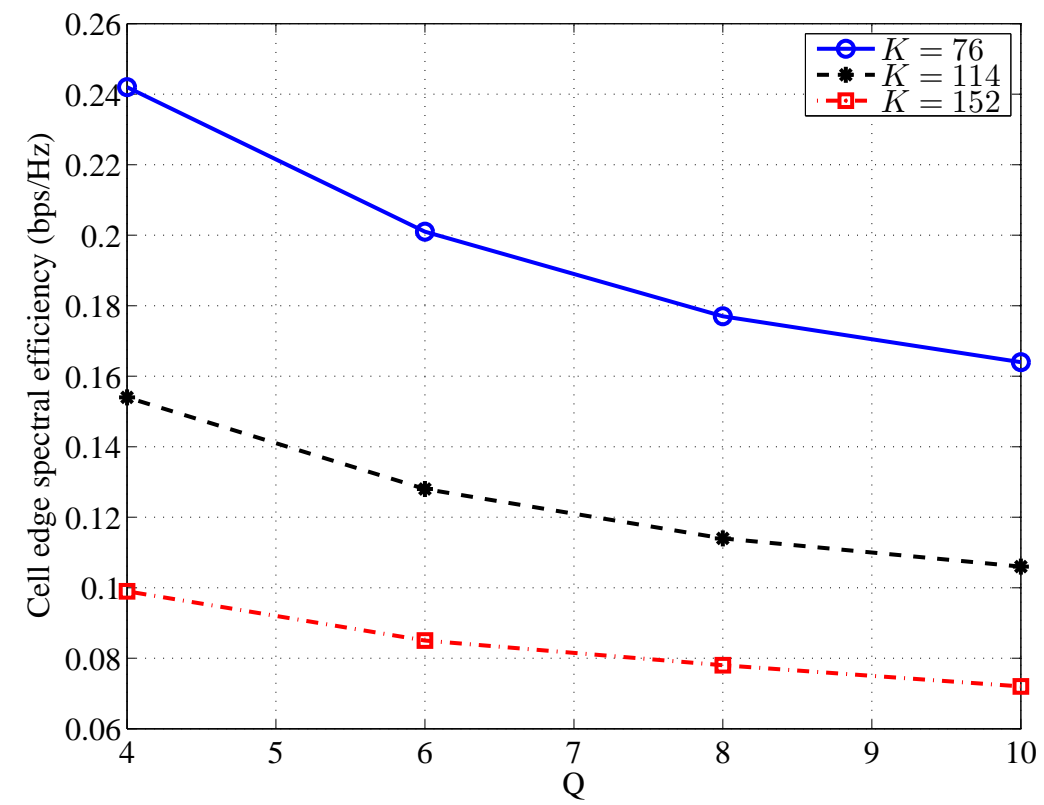

Figure 4.21: Cell edge spectral efficiency vs. the clustering parameter $Q$ for different total number of terminals in the network 
are also provided. With a $Q$ of 8 (an average scheduling cell size of $1.7 \mathrm{BSs}$ ), the cell spectral efficiency is on average $4 \%$ higher than that of the UFR technique. Increasing the coordination to between an average of $3.85 \mathrm{BSs}$ (with $Q$ of 4 ) the cell spectral efficiency improvement increases to $11 \%$.

The autonomous network is formed in an organic fashion from the irregular deployment of BSs across the network coverage area. The irregularity of the BS deployment inevitably results in a penalty in performance when compared to a regular network with the same number of BSs in the same coverage area. The performance evaluation shows that the autonomous network with no coordination (UFR) incurs a penalty of $14 \%$ in the cell spectral efficiency. The proposed framework with a $Q$ of 8 reduces this penalty to $8 \%$ which is further reduced to $3 \%$ with a $Q$ of 4 . In other words, by introducing coordination between an average of $3.5 \mathrm{BSs}$ in the network, $97 \%$ of the cell spectral efficiency of a regular network can be achieved in an autonomous network with a completely random deployment of BSs.

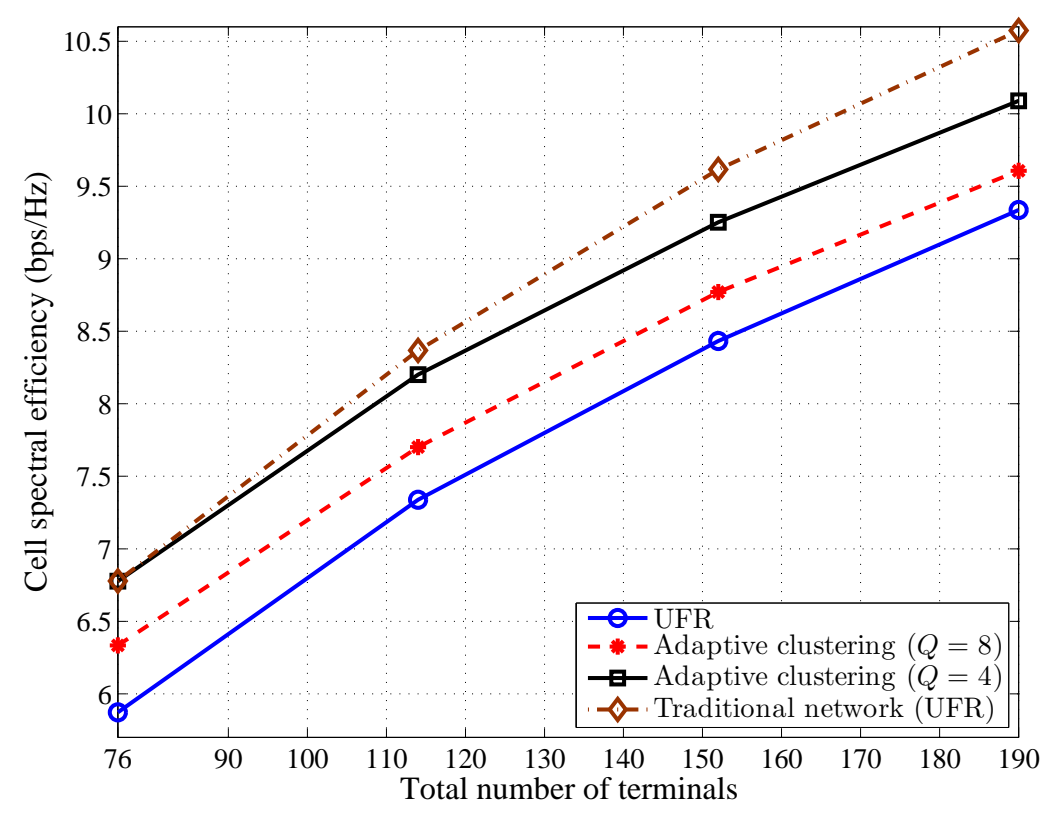

Figure 4.22: Cell spectral efficiency vs. the total number of terminals in the network

The cell edge spectral efficiency performance results are provided in Figure 4.23. The 
proposed framework with a $Q$ of 8 , improves the cell edge spectral efficiency by $17 \%$ when compared to the UFR method in the autonomous network. With a $Q$ of 4 an improvement of $54 \%$ is observed in the cell edge spectral efficiency. When compared to a traditional network, the proposed framework with a $Q$ of 8 achieves $72 \%$ of the cell edge spectral efficiency and with $Q$ equal to $4,94 \%$ of the cell edge spectral efficiency of the traditional network is achieved. It should be noted that the autonomous network with no coordination (UFR) achieves $61 \%$ of the cell edge spectral efficiency of the traditional network. Table 4.3 reports the performance evaluation results for the UFR, the proposed framework with $Q=4,8$ as a percentage of the performance in the traditional network.

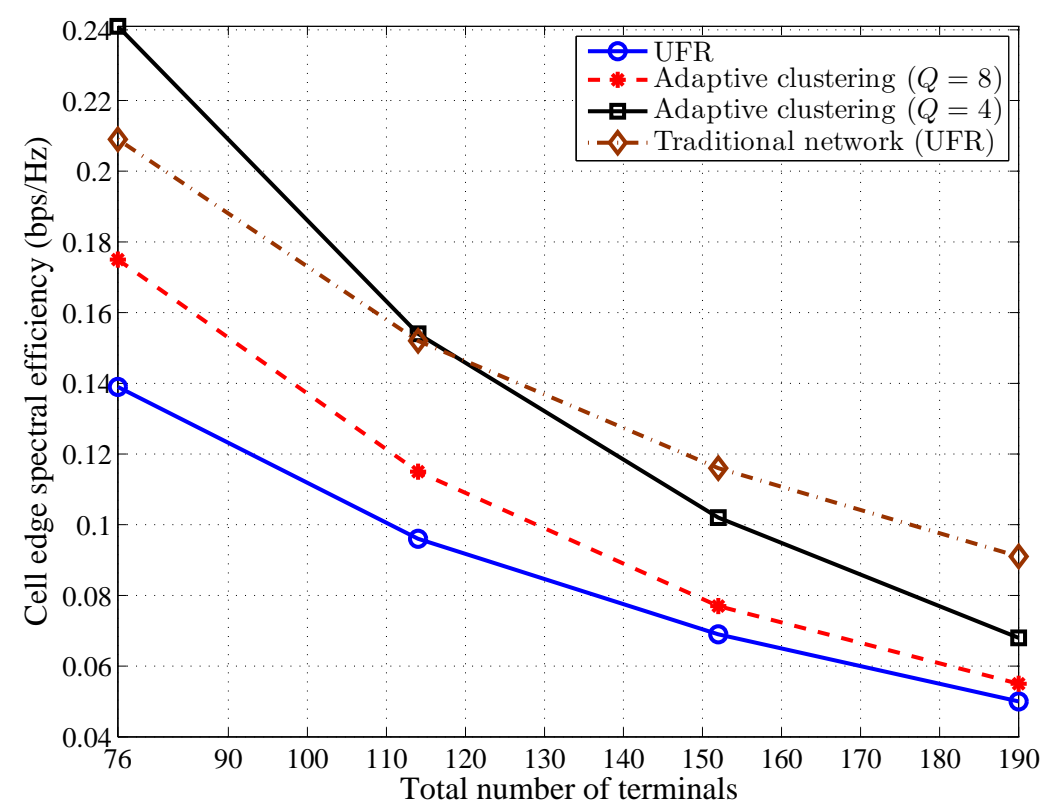

Figure 4.23: Cell edge spectral efficiency vs. the total number of terminals in the network

Table 4.3: Resource allocation performance comparison

\begin{tabular}{|c|c|c|}
\hline $\begin{array}{c}\text { Resource allocation } \\
\text { method }\end{array}$ & $\begin{array}{c}\text { Cell spectral } \\
\text { efficiency }(\%)\end{array}$ & $\begin{array}{c}\text { Cell edge spectral } \\
\text { efficiency }(\%)\end{array}$ \\
\hline \hline UFR & 86 & 61 \\
\hline Proposed $(Q=8)$ & 92 & 72 \\
\hline Proposed $(Q=4)$ & 97 & 94 \\
\hline
\end{tabular}




\section{Chapter 5}

\section{Conclusion}

\subsection{Summary}

In this thesis, we have developed adaptive frameworks for the resource allocation in cellular networks. The traditional and the autonomous network have been studied. The key difference between the two networks lies in the difference between their BS deployment patterns. While in the former the BSs are deployed in a fairly regular pattern, in the latter the BSs are irregularly deployed based on the actual traffic distribution pattern possibly by the end-users themselves.

A scheduling cell is defined by a set of coordinating BSs. In the traditional network, the scheduling cells are formed based on the regular deployment and sectorization pattern of the BSs. They are static and are comprised of the same number of BSs. In each scheduling cell, the terminals are partitioned into two sets according to their channel conditions. The system resources are assigned to the defined user sets based on their traffic demand. Terminal scheduling is performed independently for each user set on the assigned resource sets once per transmission time interval. The proposed resource allocation framework is localized. In other words, the amount of system resources assigned to the BSs vary based on the traffic demand of their assigned terminals. This results in 
a no longer regular frequency resource distribution across the BSs according to a static pre-defined pattern — as is the case in the classical method for resource allocation in cellular networks. This results in a not perfectly orthogonal resource allocation, specifically at the boundaries between the coverage areas of two adjacent BSs. Nevertheless, the performance evaluation shows that the localized resource allocation (at the expense of a partial loss of orthogonality) results in considerable improvements in terms of the achievable cell and cell edge spectral efficiencies. In addition, although the scheduling cells are static, the proposed framework is capable of adapting to the time variations in the network.

In the second part of the thesis, a cellular network with an arbitrary BS deployment pattern is considered. A network clustering method is proposed. Accordingly, the scheduling cells are formed based on the network infrastructure (i.e. distribution of the BSs and the terminals). The scheduling cells adapt to the time variations in the network. The size of each scheduling cell is a function of the density of the BS and terminal deployment in each locality. In addition, the average size of the scheduling cells is tunable in order to achieve the desirable balance between performance and complexity. A coordinated resource allocation scheme is developed which achieves proportional fairness among the terminals in each scheduling cell.

In the end, a network shadow fading model is developed. A shadow fading potential field is constructed which is essentially a two-dimensional correlated Gaussian random field. On this field, there exists an associated potential level for each terminal/BS. Given the shadow fading potential levels of two points, a channel gain generating function calculates the shadow fading gain of the link between the two points. By introducing an underlying potential field, all generated shadow fading channel gains will be correlated. The developed model is employed for the performance evaluation of the autonomous network. 


\subsection{Future work}

- In an autonomous cellular network, the deployment of extra BSs would naturally improve the system performance. If we assume there are a limited number of extra BSs to be deployed, where is the best location to deploy them in order to enhance the system performance? The strategic positioning of the extra BSs given the available network infrastructure is an interesting problem to pursue.

- In this work, all terminals are assumed to have the same quality of service (QoS) requirements. In this case, the volume of traffic can easily be translated into the number of active terminals. The resource set allocation method in Chapter 3 and the network clustering method in Chapter 4 were developed based on this assumption. The frameworks can be extended to cases where multiple classes of terminals with different QoS requirements are considered.

- We have assumed that each terminal communicates with only one BS. Alternatively, each terminal can communicate with more than one BS in the scheduling cell. Coordinated transmission from the BSs in a cell to each terminal can potentially improve the system performance. However, whether the additional complexity would result in considerable improvements or not should be carefully examined.

- Throughout the thesis, the power budget of each BS is assumed to be equally distributed across the available frequency channels. The frequency channels are then assigned based on a terminal scheduling policy. Although this appears to be the philosophy behind the design of the OFDMA air interface technology, power control is still an area which should be thoroughly studied in the autonomous cellular networks. 


\section{Bibliography}

[1] 3GPP TS 36.213: Evolved Universal Terrestrial Radio Access (E-UTRA); Physical layer procedures.

[2] ITU-R M.2135, Requirements related to technical performance for IMT-advanced radio interface(s), 2008.

[3] S. H. Ali and V. C. M. Leung. Dynamic frequency allocation in fractional frequency reused OFDMA networks. IEEE Transactions on Communications, 8(8):4286-4295, August 2009 .

[4] W. Anchun, X. Liang, Z. Shidong, X. Xibin, and Y. Yan. Dynamic resource management in the fourth generation wireless systems. In Proceedings of International Conference on Communication Technology, pages 1095-1098, April 2003.

[5] G. Aniba and S. Aissa. Adaptive proportional fairness for packet scheduling in HSDPA. In Proceedings of IEEE Global Communications Conference, pages 40334037, November 2004.

[6] P. Bender, P. Black, M. Grob, R. Padovani, N. Sindhushyana, and S. Viterbi. CDMA/HDR: a bandwidth efficient high speed wireless data service for nomadic users. IEEE Communications Magazine, 38(7):70-77, July 2000.

[7] T. Bonald, S. Borst, and A. Proutière. Inter-cell scheduling in wireless data networks. European wireless proceedings, 2005. 
[8] J. Y. Le Boudec. Rate adaptation, congestion control and fairness: a tutorial. Ecole Polytechnique Federale de Lausanne, 2008.

[9] G. Boudreau, J. Panicker, N. Guo, R. Chang, N. Wang, and S. Vrzic. Interference coordination and cancellation for $4 \mathrm{G}$ networks. IEEE Communications Magazine, 47(4):74-81, April 2009.

[10] V. Chandrasekhar, J. Andrews, and A. Gatherer. Femtocell networks: a survey. IEEE Communications Magazine, 46(9):59-67, September 2008.

[11] R. Y. Chang, Z. Tao, J. Zhang, and C.-C. J. Kuo. Multicell OFDMA Downlink Resource Allocation Using a Graphic Framework. IEEE Transactions on Vehicular Technology, 58(7):3494-3507, September 2009.

[12] H. Dahrouj and W. Yu. Interference Mitigation with Joint Beamforming and Common Message Decoding in Multicell Systems. In Proceedings of IEEE International Symposium on Information Theory, June 2010.

[13] B. Golkar and E. S. Sousa. A generalized methodology for frequency reuse in autonomous cellular networks. In Proceedings of IEEE International Symposium on Personal, Indoor and Mobile Radio Communications, September 2011.

[14] B. Golkar and E. S. Sousa. Adaptive localized resource allocation with access point coordination in cellular networks. In Proceedings of IEEE International Conference on Communications, June 2011.

[15] B. Golkar and E. S. Sousa. A network shadow fading model for autonomous infrastructure wireless networks. In Proceedings of European Signal Processing Conference, August 2012. 
[16] B. Golkar and E. S. Sousa. Adaptive partitioned interference management in cellular networks. In Proceedings of IEEE Global Communications Conference, December 2012.

[17] M. Gudmundson. Correlation model for shadow fading in mobile radio systems. Electronics Letters, 27(23):2145-2146, November 1991.

[18] S. W. Halpern. Reuse partitioning in cellular systems. In Proceeding of IEEE Vehicular Technology Conference, June 1983.

[19] J. Jang and K. B. Lee. Transmit power adaptation for multiuser OFDM systems. IEEE Journal on Selected Areas in Communications, 21(2):171-178, February 2003.

[20] M. Kaneko, P. Popovski, and J. Dahl. Proportional fairness in multicarrier system: upper bound and approximation algorithms. IEEE Communications Letters, 10(6):462-464, June 2006.

[21] I. Katzela and M. Naghshineh. Channel assignment schemes for cellular mobile telecommunication systems: a comprehensive survey. IEEE Personal Communications, 3(3):10-31, August 1996.

[22] F. Khan. LTE for 4G Mobile Broadband: Air Interface Technologies and Performance. Cambridge University Press, 2009.

[23] H. Kim and Y. Han. A proportional fair scheduling for multicarrier transmission systems. IEEE Communications Letters, 9(3):210-212, March 2005.

[24] H. Kim, K. Kim, Y. Han, and J. Lee. An efficient algorithm for QoS in wireless packet data transmission. In Proceedings of IEEE International Symposium on Personal, Indoor and Mobile Radio Communications, pages 2244-2248, April 2002.

[25] K. Kumaran, S. E. Golowich, and S. Borst. Correlated shadow-fading in wireless networks and its effect on call dropping. Wireless networks, 8(1):61-71, 2002. 
[26] G. Li and H. Liu. Downlink Radio Resource Allocation for Multi-Cell OFDMA System. IEEE Transactions on Wireless Communications, 5(12):3451-3459, December 2006.

[27] D. Lopez-Perez, A. Valcarce, G. de la Roche, and J. Zhang. OFDMA femtocells: A roadmap on interference avoidance. IEEE Communications Magazine, 47(9):41-48, September 2009.

[28] V. H. Macdonald. The cellular concept. Bell System Technical Journal, 58(1):15-41, 1979.

[29] M. Rahman and H. Yanikomeroglu. Enhancing cell-edge performance: a downlink dynamic interference avoidance scheme with inter-cell coordination. IEEE Transactions on Wireless Communications, 9(4):1414-1425, April 2010.

[30] T. S. Rappaport. Wireless Communications: Principles $\mathscr{E}$ Practice. Prentice Hall, NJ, 2002.

[31] K. Son, Y. Yi, and S. Chong. Utility-optimal multi-pattern reuse in multi-cell networks. IEEE Transactions on Wireless Communications, 10(1):142-153, January 2011.

[32] G. Song and Y. Li. Cross-layer optimization for OFDM wireless networks-part II: algorithm development. IEEE Transactions on Wireless Communications, 4(2):625634, March 2005.

[33] L. Venturino, N. Prasad, and X. Wang. Coordinated scheduling and power allocation in downlink multicell OFDMA networks. IEEE Transactions on Vehicular Technology, 58(6):2835-2848, 2009.

[34] U. von Luxburg. A tutorial on spectral clustering. Statistics and computing, 17(4):395-416, August 2007. 
[35] L. Yang and M.-S. Alouini. Performance analysis of multiuser selection diversity. IEEE Transactions on Vehicular Technology, 55(6):1848-1861, November 2006.

[36] L. Yang, M. Kang, and M.-S. Alouini. On the capacity-fairness tradeoff in multiuser diversity systems. IEEE Transactions on Vehicular Technology, 56(4):1901-1907, July 2007.

[37] W. Yu. Multiuser waterfilling in the presence of crosstalk. In Information Theory and Applications Workshop, pages 414-420, January 2007.

[38] W. Yu, T. Kwon, and C. Shin. Adaptive resource allocation in cooperative cellular networks. In Cooperative cellular wireless networks, pages 233-256. Cambridge University Press, 2011. 